University of Redlands

\title{
Reconstructing the Past in 3D Using Historical Aerial Imagery
}

A Major Individual Project submitted in partial satisfaction of the requirements for the degree of Master of Science in Geographic Information Systems

by

Michael Broadbent

Ruijin Ma, Ph.D., Committee Chair

Mark Kumler, Ph.D.

December 2017 
Reconstructing the Past in 3D - Using Historical

Aerial Imagery

Copyright (C) 2017

by

Michael Broadbent 
The report of Michael Broadbent is approved.

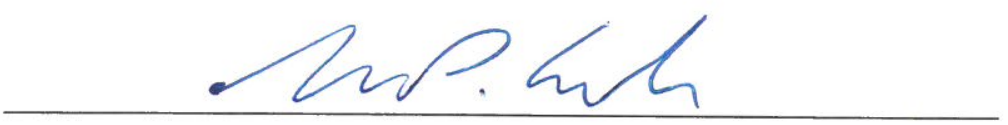

Mark Kumler, Ph.D.

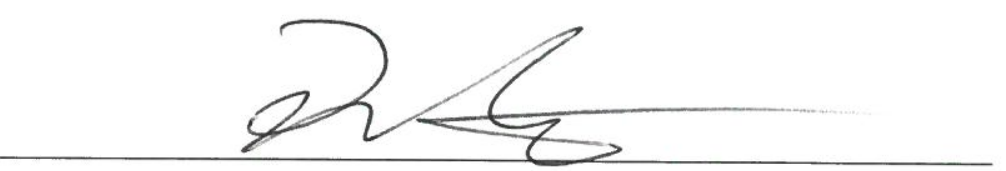

Ruijin Ma, Ph.D., Committee Chair

December 2017 



\section{Acknowledgements}

I would like to thank Jack and Linda Dangermond for sponsoring the Esri Fellowship program. It is a tremendous initiative and without it I would not have taken the opportunity to pursue further higher education.

Thank you to my advisor Dr. Ruijin Ma who provided me a new direction and project when my first one fell through. I really appreciate your efforts to provide guidance during the project and efforts to position me for success.

Thank you as well to all the staff and faculty at the University of Redlands MS GIS program. It has been a wild ride and it was a pleasure to learn with you.

Lastly, but most importantly thanks to my wife Aimee Broadbent and children Jasper, Westley, and Amelia for their love and support over the last two years. It has not always been the easiest journey. I appreciate your patience, encouragement, and effort to make it work even in the hardest times. 



\author{
Abstract \\ Reconstructing the Past in 3D - Using Historical Aerial Images \\ by \\ Michael Broadbent \\ Historical aerial film images are a valuable record of the past, and are useful as a baseline \\ for change detection and landcover analysis. To be useful in GIS analysis the images \\ must be oriented to a spatial reference system. This is challenging as historical imagery is \\ often missing flight and camera information. Traditional photogrammetric processing \\ techniques exist to overcome these challenges, but they require specialized knowledge, \\ time and expense to complete. Because of this, many collections of historical images are \\ This project produced a method to quickly standardize the photos, spatially orient \\ them, correct them for distortion effects, and extract a digital surface model from the \\ overlapping image series using Pix4D Professional. The horizontal accuracy met \\ National Map Accuracy Standards when the Pix 4D process was combined with \\ traditional georeferencing. The workflow was faster than traditional methods due to
} left unprocessed. economies of scale in the new process. 



\section{Table of Contents}

Chapter 1 - Introduction ...................................................................................................... 1

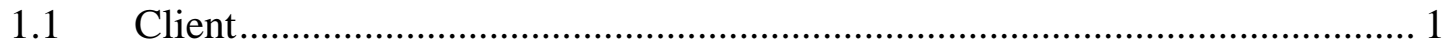

$1.2 \quad$ Problem Statement .......................................................................... 2

$1.3 \quad$ Proposed Solution ........................................................................... 2

1.3.1 Goals and Objectives ............................................................................ 2

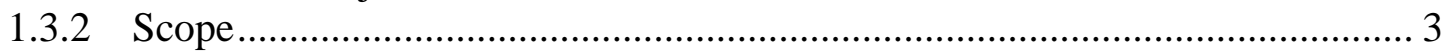

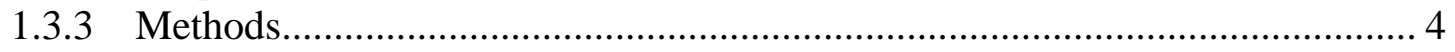

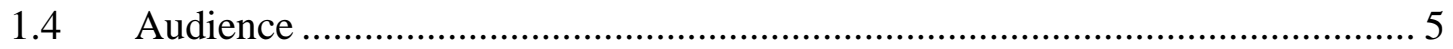

1.5 Overview of the Rest of this Report ................................................................. 5

Chapter 2 - Background and Literature Review ............................................................ 7

$2.1 \quad$ Historical Aerial Photography in Geographic Analysis..................................... 7

$2.2 \quad$ Error Sources of Aerial Photographs ............................................................. 8

2.2.1 Interior Orientation Parameters.................................................................... 9

2.2.2 Exterior Orientation Parameters ................................................................... 10

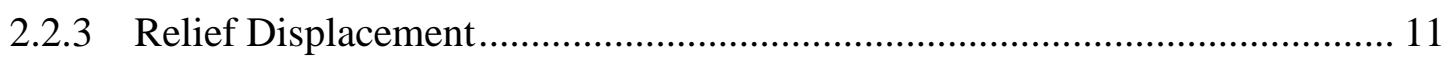

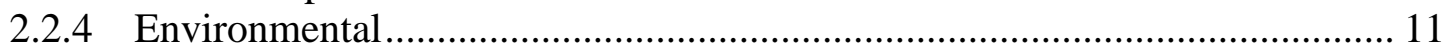

2.2.5 Scanning Film Aerial Imagery .......................................................................... 11

2.3 Spatial Referencing and DSM generation from Aerial Images ....................... 12

2.4 Computer Vision and Structure from Motion ................................................. 13

2.4.1 Detailed Methodology …………………………........................................... 14

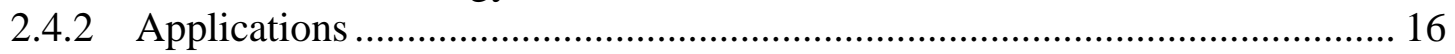

2.4.3 SfM to Historical Imagery …………………….......................................... 17

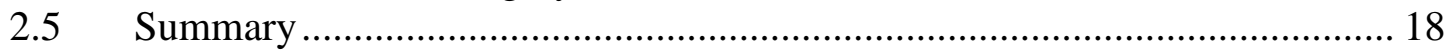

Chapter 3 - Systems Analysis and Design........................................................................ 19

3.1 Problem Statement ...................................................................................... 19

3.2 Functional and Non-Functional Requirements Analysis .................................. 19

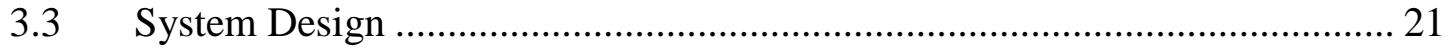

3.4 Project Plan ...................................................................................... 23

3.5 Summary ............................................................................................. 26

Chapter 4 - Database Design.............................................................................................. 27

$4.1 \quad$ Conceptual Data Model ......................................................................... 27

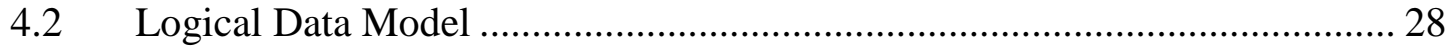

4.3 Data Sources ........................................................................................ 30

4.4 Data Collection Methods ............................................................................... 31

$4.5 \quad$ Data Scrubbing and Loading …………………......................................... 31

$4.6 \quad$ Summary .......................................................................................... 31

Chapter 5 - Implementation......................................................................................... 33

$5.1 \quad$ Image Standardization ............................................................................... 33

$5.2 \quad$ Image Center Coordinates............................................................................... 35

5.3 Reference Control Points ........................................................................ 36

$5.4 \quad$ Generate Orthophoto and DSM .................................................................. 38

5.4.1 Phase 1 - Initial Processing ………………………......................................... 39 


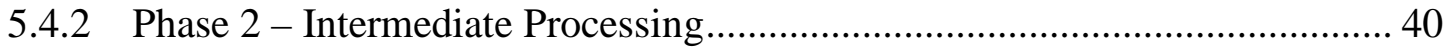

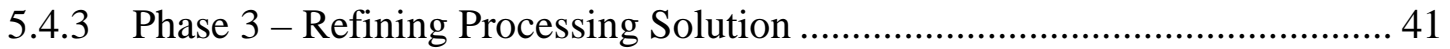

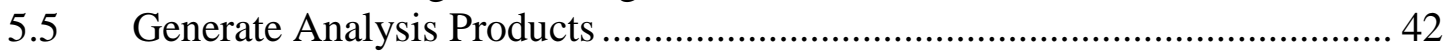

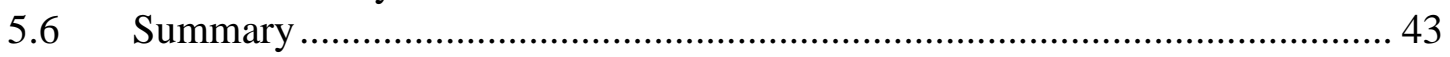

Chapter 6 - Results and Analysis.................................................................... 45

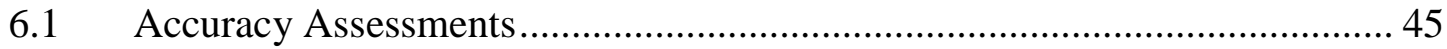

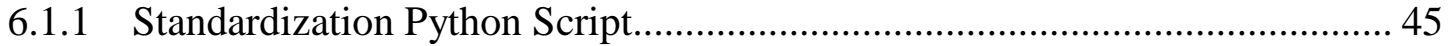

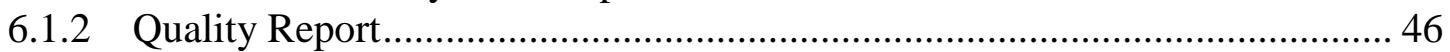

6.1.3 Orthorectified Image Mosaic .................................................................. 48

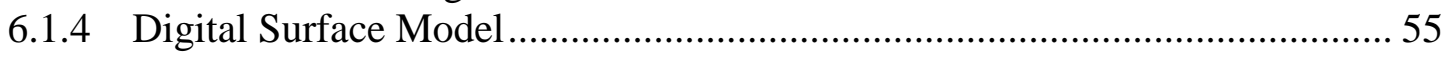

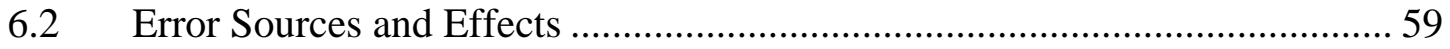

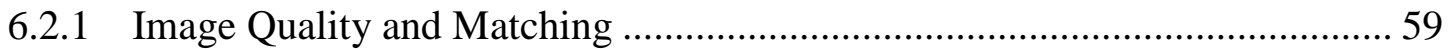

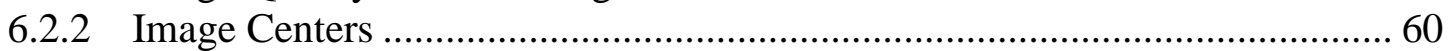

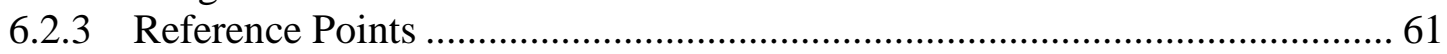

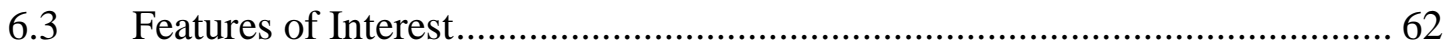

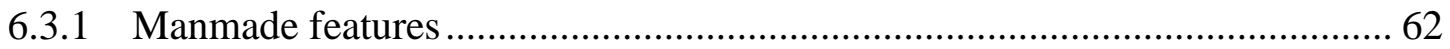

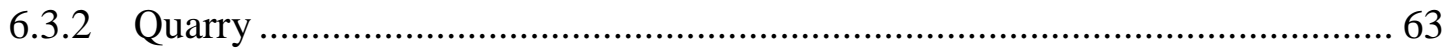

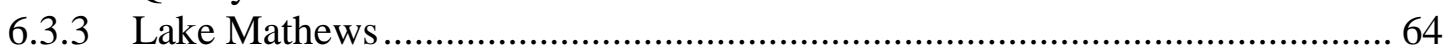

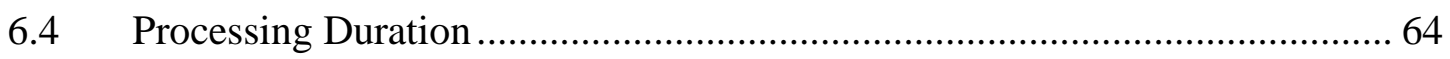

Chapter 7 - Conclusions and Future Work ........................................................66

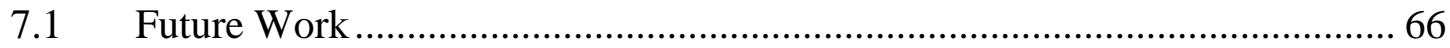

Works Cited ....................................................................69

Appendix A. Standardization Script Analysis .......................................................... 71

Appendix B. Analysis and Quality Reports................................................................ 73

Appendix C. Python Image Standardization Script .................................................. 94 


\section{Table of Figures}

Figure 1-1: Project area around the city of Riverside, California................................ 3

Figure 2-1: Pop Laval - Image courtesy of Pop Laval Foundation.............................. 8

Figure 2-2: Aerial photograph showing the location of three fiducial marks.............9

Figure 2-3: Tilted photograph showing image skew …....................................... 10

Figure 2-4: Vertical photograph showing relief displacement ................................ 11

Figure 2-5: Hybrid Computer Vision SfM photogrammetry process........................15

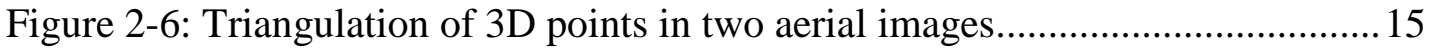

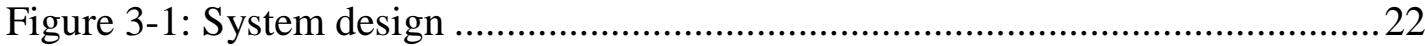

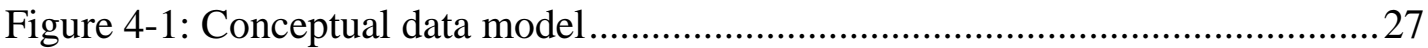

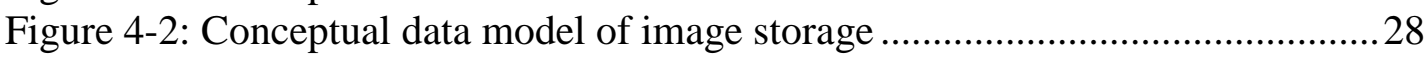

Figure 4-3: Logical data model.........................................................................29

Figure 5-1: Python script image standardization process ......................................33

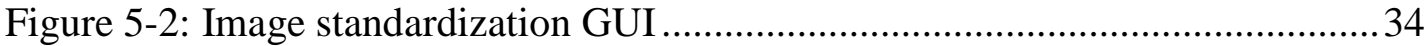

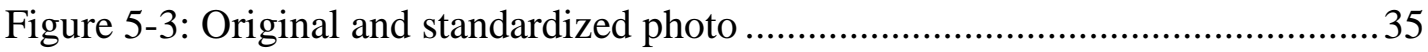

Figure 5-4: Correlating common features of a historical photo to present day ..........36

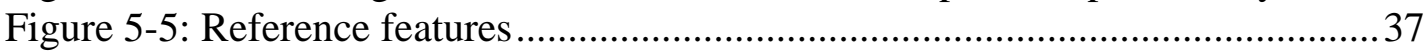

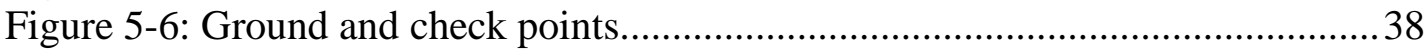

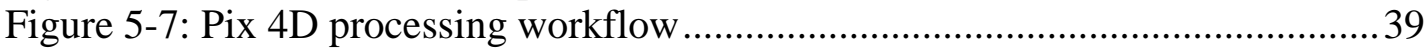

Figure 5-8: Tagging control points in imagery ................................................ 40

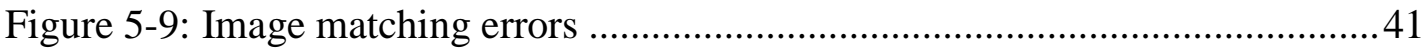

Figure 5-10: Elevation spikes caused by image mismatches...................................41

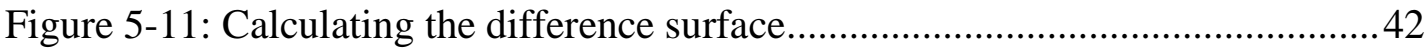

Figure 6-1: Image overlap of the project area....................................................... 47

Figure 6-3: Matched keypoints from three or more images ...................................49

Figure 6-4: USGS basemap (left) and processed orthomosaic (right) .....................49

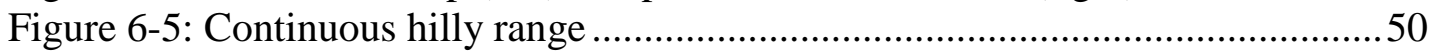

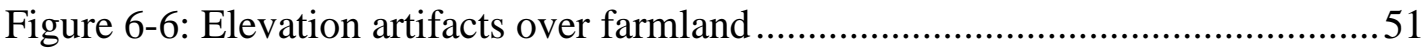

Figure 6-7: Horizontal residuals (left) and elevation difference (right) ....................5 52

Figure 6-8: Georeferenced orthomosaic .................................................................. 53

Figure 6-9: Horizontal residuals (left) and elevation difference using more control points

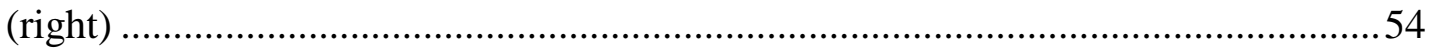

Figure 6-10: Digital surface model with hillshade overlay ....................................56

Figure 6-11: Computed DSM (left) and difference between surface between DSM and

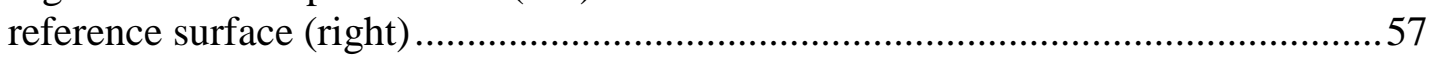

Figure 6-12: Effects of adding manual tie points on elevation artifacts .................... 58

Figure 6-13: Elevation artifacts isolated and corrected using the Raster Calculator in

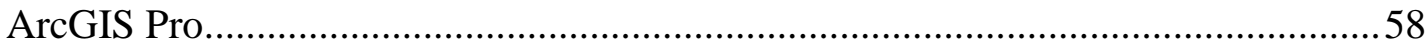

Figure 6-14: Project flight lines and image centers ..............................................59

Figure 6-15: Differences in overlapping image quality .......................................6 60

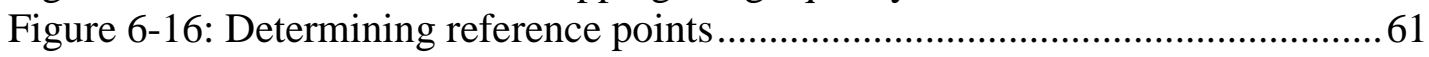

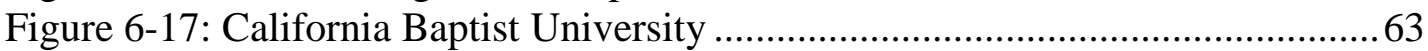

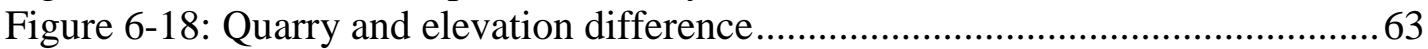

Figure 6-19: Lake Mathews orthomosaic and DSM with hillshade .........................64 


\section{List of Tables}

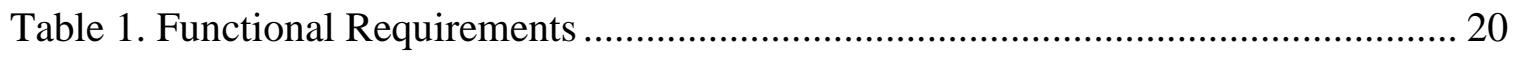

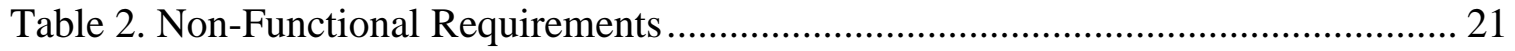

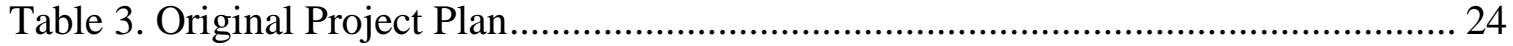

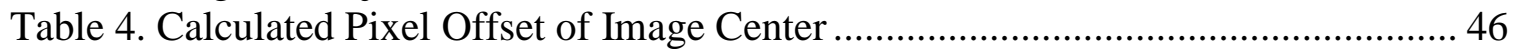

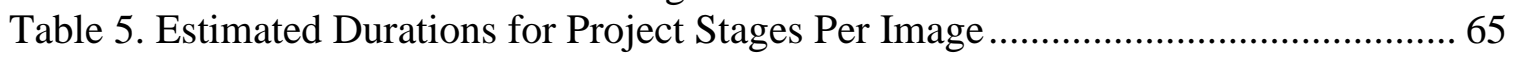




\title{
List of Acronyms and Definitions
}

\author{
CCB - Center for Conservation Biology \\ DEM - Digital Elevation Model \\ DSM - Digital Surface Model \\ DPI - Dots Per Inch \\ GSD - Ground Sampling Distance \\ GUI - Graphical User Interface \\ MVS - Multi-View Stereo \\ NAD - North American Datum \\ RMSE - Root Mean Square Error \\ SfM - Structure from Motion \\ UAV - Unmanned Aerial Vehicle \\ USDA - United States Department of Agriculture \\ USGS - United States Geological Survey \\ UTM - Universal Transverse Mercator
}





\section{Chapter 1 - Introduction}

In the Great Depression of the 1930s food prices fell due to a combination of low demand and over production. To stabilize them, Congress created farming programs where farmers agreed to curtail production or switch to different crops in return for compensation from the government (Monmonier, 2002). To determine the compensation and monitor the effectiveness of the program Congress requisitioned large aerial photography campaigns over much of the United States (Monmonier, 2002). The purpose of the aerial acquisitions was to determine the total acreage of each farm and the effective acreage sown with crops.

On May $24^{\text {th }}$, 1938, the renown photographer Pop Laval was awarded a contract with the United States Department of Agriculture (USDA) to acquire aerial photography over much of Riverside county, California (University of California, 2012). This imagery was preserved as a historical reference by the Center for Conservation Biology (CCB) and stored at the library of the University of California, Riverside. It has frequently been used as a visual baseline from the past for research.

Like many historical aerial photography collections, the images are an extremely valuable historical record, and useful in such applications as change detection and landcover analysis. While these film-based photographs have been scanned into a digital format, the digital scans were missing spatial reference information to orient them in the world, and correct them for effects such as image distortion, tilt, and relief displacement. The original images also offered no information to the viewers about the terrain morphology or elevation in the image. As uncorrected raw imagery, they had little GIS analytical value. To process these images into relevant resources was slow and costly.

This project produced a method to quickly standardize and prepare the historical aerial photos, spatially orient and correct them for distortion effects, and extract a terrain surface from the overlapping image series using triangulation. The project used traditional photogrammetric techniques combined with modern computer vision processing algorithms known as Structure from Motion (Sfm) to self-calibrate and automate image triangulation and orientation using Pix4D Professional. A Digital Surface Model (DSM) of the terrain and an orthorectified image mosaic was created from the overlapping images. Key features of interest in the project area were also identified. A workflow was also produced to aid the CCB or other institutions with processing their image collections to create information products that could be used in GIS processing and analysis.

\subsection{Client}

The client for this project was Dr. Ruijin Ma, Associate Professor of GIS at the University of Redlands, California. Professor Ma served as the advisor to an earlier project that used a Rational Function Model to orthorectify the same scanned aerial imagery collection (Buchwald, 2011). The project was a success, but the method developed required too much manual interaction per image by the CCB. Because of the time and cost, they were not able to scale up the method for use on their entire image collection. Professor Ma wanted to evaluate whether computer vision photogrammetric 
processing technology could provide a similar outcome, with much less manual interaction, and create new information products.

While the CCB was not the direct client for this project, Robert Johnson, Assistant Specialist in GIS for the CCB, did provide supplementary support and resources on the background of the aerial photography and the previous project requirements and expectations. Janet Reyes, Geospatial Information Librarian from the University of California, also provided support and resources.

\subsection{Problem Statement}

Historical aerial images are a valuable visual record of the past, and are useful as a baseline for change detection and landcover analysis. Many collections of historical film images sit untouched in archives; the one collection studied for the CCB contains over 600 aerial images over Riverside County, California. Scanning the imagery preserves them as a visual reference, but their value in GIS analysis is limited because they are not oriented and positioned in the world. The images need to be processed using photogrammetric techniques to correct them for orientation and distortion errors, and to extract additional data products like terrain surfaces.

Correcting the imagery is challenging because historical aerial image collections like the one used in this project are often missing details about the camera used in the acquisition and the flight parameters. This limits the types of processing techniques available for use to ones that are manually time intensive, slow, and costly to perform. These drawbacks often leave the archived images unprocessed and left as only a visual reference for research and analysis.

\subsection{Proposed Solution}

The CCB needed a method to process its archived aerial imagery collection efficiently with as little manual interaction or specialized knowledge as possible. The intention was to create a solution that could spatially orient the images and correct them for common errors while creating additional information products like a surface model. After an evaluation of the project requirements, the source imagery, and the supporting project information, a semi-automated triangulation approach using overlapping images in series was selected. The approach uses traditional photogrammetric processing combined with computer vision Structure from Motion (SfM) techniques to produce a robust solution that can self-calibrate and overcome the challenges of unknown uncalibrated cameras, inaccurate GPS systems and environmental conditions (Eisenbeib, 2009). This technique was created out of necessity due to the rapid increase in image acquisition rates from various camera sensors like those on Unmanned Aerial Vehicles (UAVs). It is now available in commercial photogrammetry software like Pix4D Professional.

\subsubsection{Goals and Objectives}

The goal of this project was to provide a solution to easily orthorectify historical aerial imagery to an accuracy that met the National Map Accuracy Standards even in areas where there was dramatic change in elevation. Additionally, it would derive a DSM of 
the project site's terrain from the imagery and identify key features that had changed over time in the project area. The specific objectives of this project were to:

- Create a Python script to standardize the input scanned aerial imagery, so that each image had an identical image center, orientation and size.

- Create a seamless orthorectified image mosaic of the sample area around the City of Riverside generated from the historical aerial imagery. The mosaic would target meeting the National Map Standards for image accuracy, and have a $2 \mathrm{~m}$ cell size and a spatial reference frame of NAD83 UTM Zone 11.

- Create a digital surface model of the sample area around the City of Riverside generated from the triangulation of aerial imagery points. The DSM would have a desired vertical accuracy of $5 \mathrm{~m}$ over surfaces determined to be permanent for the last 80 years. The DSM would be cleaned of obvious erroneous elevation data, and be $2 \mathrm{~m}$ resolution with NAD83 UTM Zone 11 as a spatial reference.

- Establish a workflow to process historical aerial image processing to generate the information products described above.

\subsubsection{Scope}

There were over 600 black and white aerial images in the CCB collection for the County of Riverside, California, that were taken as part of the 1938 acquisition campaign. Due to the time constraints of this project it was not possible to process them all, so a selection of thirty images from five separate flight lines was selected that covered an area in and around the City of Riverside, as illustrated in red in Figure 1-1 below.

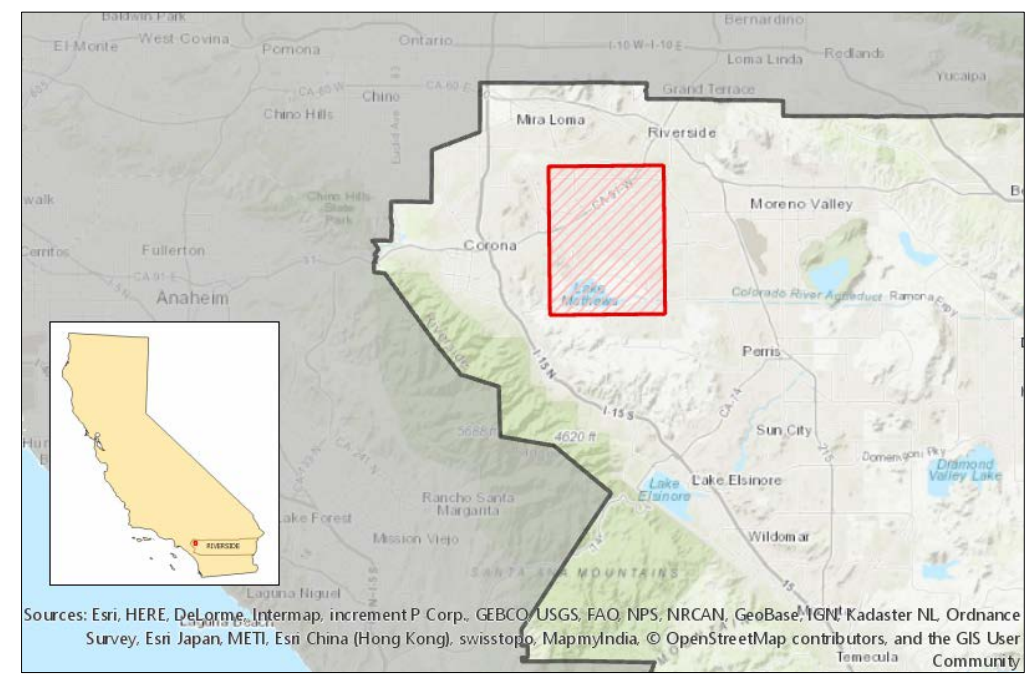

Figure 1-1: Project area around the city of Riverside, California

This area was selected because of the variety of landcover types available and because over 80 years there had been a significant conversion from rural to urban in and around the city. The site also offered a combination of flat terrain and mountainous areas to the south. Lastly, the target project area overlapped with Lake Mathews, a manmade lake to the south. In 1938 this lake was still under construction and had not yet been 
filled; it represented a significant known feature that had changed from the past to present day.

Because the processing methodology relied on processing many overlapping series of photos at a time, it was necessary to find an area with many different terrain characteristics. The selected images represented a pilot program for the overall county collection that could then be adapted and applied to the rest of the county. The deliverables were static imagery based products, so they could easily be used in subsequent mapping or spatial analysis workflows as desired.

\subsubsection{Methods}

An initial review in the project planning phase created the requirements and defined a timeline for completion. From that analysis, the project stages were defined and included: data review and standardization, image center and control point acquisition, data processing, data cleaning, and final product compilation. A summary of the methods of each stage are referenced below, and will be described in full in later sections.

The imagery for the pilot project area was obtained from the University of California, Riverside Library. There was no camera calibration report available that contained the interior orientation parameters of the camera. The scanned imagery was raw and every image was scanned slightly differently so a Python script was written to standardize the images. The script allowed users to pick fiducial marks on each image programmatically to obtain their coordinates. It then automatically computed the image center and rotated the images to be orientated correctly, and finally the script cropped them to a standard image size and exported them. The standardization ensured that after the processing software performed a self-calibration on the imagery, the resulting corrections would be applied consistently across all images.

Once the image center for each image was determined, it was positioned in the real world using current reference imagery obtained from the USGS. This correlation process occurred visually in ArcGIS Pro. Each center point was captured into a point feature class stored in a file geodatabase. The elevation for the image center was computed using a reference DEM, publicly available from the USGS, and an approximation of the flying height. Similarly, ground control and check points were identified between the source and reference images representing stable unchanged features common on both datasets and their elevation extracted from the same DEM. Project data were referenced to NAD83 and UTM Zone 11 North, for Riverside, California.

Once the imagery was standardized and control information obtained the imagery was processed in Pix4D Professional. Pix4D is a software company that specializes in photogrammetric software that employ computer vision processing techniques. The initial image center coordinates were used to help the image matching process in areas of high elevation change. The triangulation process computed coordinates for matched points between images. After initial processing, ground control points were imported and applied to the solution to increase the positioning accuracy. The processing results were checked against check points to provide a relative accuracy assessment. Finally, manual tie points were created in Pix4D Professional to eliminate erroneous elevation artifacts in the data created through the automated image triangulation and matching process. To meet the National Map Accuracy Standards the Pix 4D process was combined with traditional georeferencing to meet the target horizontal accuracy. 
The resulting orthorectified image mosaic and DSM of the project area were saved into a file geodatabase for reference and the final mapping and analysis products were created in ArcGIS Pro. Using the raster analysis tools comparisons were created between the newly generated DSM and the reference DEM from USGS.

\subsection{Audience}

The primary audience for this project were GIS Specialists within the CCB who had photogrammetric processing experience and could guide others using the procedures defined in the processing workflow to complete the orthorectification and DSM generation for the remainder of Riverside County. There are also thousands of public and private historical aerial image collections that could be processed by adapting the techniques discussed in this project. The people who own these collections may not be familiar with GIS terminology, so the results of this project are written in such a way as to be approachable by an audience without a GIS background.

\subsection{Overview of the Rest of this Report}

The following six chapters provide a detailed analysis of the process of using computer vision photogrammetric techniques to create information products from the historical aerial imagery acquired over Riverside County in 1938. Chapter 2 provides a literature review of use cases where aerial imagery was used in landcover analysis, including the history of this particular imagery collection from 1938. It also explores different processing techniques used in image processing, and common challenges of working with aerial imagery. Chapter 3 discusses system analysis and the project requirements. Chapter 4 , reviews the database design and specifics of the data sources used on this project. Chapter 5 discusses the final implementation and the process to create the final deliverables and analysis. Chapter 6 provides the final results and discussion. Chapter 7 provides a conclusion along with some ideas for future work in this area. 



\section{Chapter 2 - Background and Literature Review}

To determine an appropriate solution to creating meaningful geographic data products using historical aerial photography required reviewing previous research and work in the field. This chapter discusses why historical aerial photographs are still relevant in geography, and some of the challenges of using them in analysis. In addition, this chapter reviews the traditional photogrammetric processing techniques that are typically applied to correct image errors, and the modern computer vision photogrammetric techniques that are growing in use today.

\subsection{Historical Aerial Photography in Geographic Analysis}

In 1858, the first aerial photograph was captured from a hot air balloon tethered to the ground in France, by Gasper Felix Tournachon (Kim \& Muller, 1996). That moment was a catalyst for a new way of collecting information: one that allowed for data to be captured over large areas, in a short amount of time, and without physical engagement in the area. This practice stayed in the realm of hobbyist until the World War I, when in 1914 the first aerial photographs were taken for military reconnaissance (Stichelbaut, 2006).

It was the advantages of remote data capture that lead the USDA to use aerial photography as a means of farm surveillance during the Great Depression (Monmonier, 2002). In the 1930s, farmers were hit hard by low prices, and to stabilize the market the government offered benefits to those who reduced their farm acreage or planted soilconserving crops (Monmonier, 2002). On site monitoring through ground traverse of the farms was impractical, so the agency experimented with aerial photography in the mid 1930s to calculate effective farm acres to 1\% accuracy (Monmonier, 2002). Field areas were computed by measuring the target area in square inches on the photographs and multiplying by a scale factor that was computed for every fifth or sixth frame (Monmonier, 2002).

The image acquisitions were successful despite some concerns about the minimal corrections applied for tilt and relief. The acquisitions continued through the 1930s and resulted in photographic coverage of much of the United States. This included the county of Riverside, California, which was acquired by Pop Laval, depicted below in Figure 2-1, and used in this research project. 


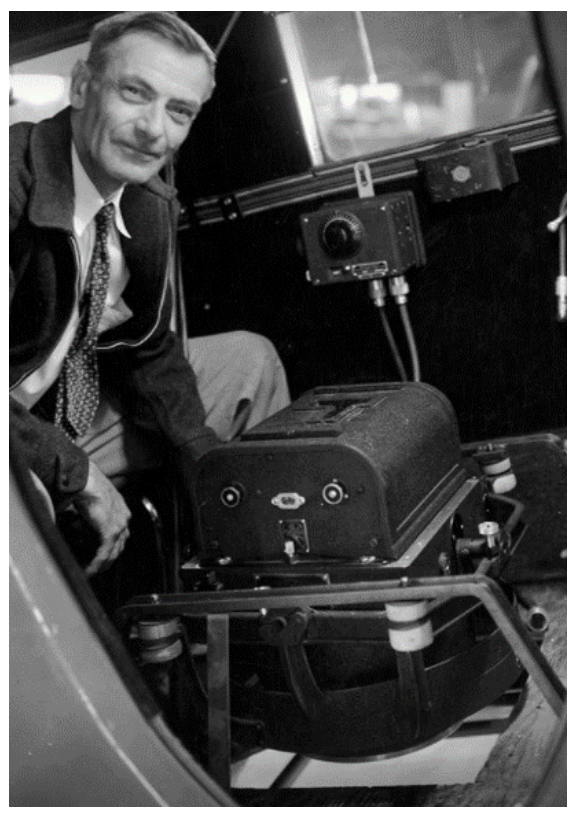

Figure 2-1: Pop Laval - Image courtesy of Pop Laval Foundation

The Riverside collection of photographs was later acquired by the library of the University of California, Riverside. It has remained as an archived collection for use as a historical reference and geographic analysis resource.

The last 100 years have seen countless technological leaps in aircraft, camera, positioning, and computer technology which have improved the quality and cost effectiveness of aerial photography in remote sensing analysis to have a positive impact on applications such as environmental monitoring, land cover analysis, urban mapping, surveying, resource management, and biological research (Morgan, Gergel, Ankerson, Tomscha, \& Sutherland, 2017). Archived aerial photography still has value, as it can "provide valuable information on prior or baseline landscape conditions, making the imagery useful for mapping and monitoring change over time” (Morgan, Gergel, Ankerson, Tomscha, \& Sutherland, 2017, p. 22).

This was illustrated by Estes, Jensen and Tinney (1977) when they used aerial photographs from the 1930s and 50s to assist in mapping sites of Californian mission sites destroyed in 1812. Using simple techniques for the time they were able to quickly correlate common features between the aerial images and planimetric maps, and then rubber sheet the image into an approximate position to define the location of each mission. This process contributed to the site obtaining historic status by California.

\subsection{Error Sources of Aerial Photographs}

The quality and uncertainty of every frame that is captured by a camera, whether it is a digital or film camera, can be "influenced by a wide variety of factors such as earth curvature, film and paper shrinkage, nonplanar image film plane, atmospheric refraction effects, optical distortions, tilt and relief displacements” (Verhoeven, et al., 2013, p. 35). The consequences of these effects vary in magnitude with changes to the camera's orientation and subject matter in each photo. 
To use film images in computing systems they must be scanned. Scanned film images suffer additional degradations resulting in the loss of "radiometric and tonal variation and spatial resolution" (Morgan, Gergel, \& Coops, 2010, p. 50) from the original image. A description of every major image error source, its effects and its impact on use in historical aerial photography analysis is described in the following sections.

\subsubsection{Interior Orientation Parameters}

A camera's interior orientation parameters are used to define the camera's internal geometry, specifically the location of the principle point and focal length of the camera, and corrections for any distortion effects (Jain, Kasturi, \& Schunck, 1995). These parameters are used together to accurately transform coordinates from an image pixel coordinate system to an image space coordinate system. The principle point location is the geometric center of the photograph. It is also the point from which the focal length of the lens is measured (Wolf \& Dewitt, 2000). The focal length, is the distance from the principle point to the perspective center. The lens distortion corrections, are broken into two types: radial and tangential lens distortion. Radial distortion refer to the effects of light bending more near the edges of an image than the center. Tangential distortion is caused by imperfections in the alignment of the camera lens with the image plane (Wolf \& Dewitt, 2000).

These parameters are calibrated in a lab by taking "target images and comparing their actual image locations with the positions they should have occupied had the camera produced a perfect perspective view" (Wolf \& Dewitt, 2000, p. 64). This comparison is performed using the image's fiducial marks, as shown in Figure 2-2.

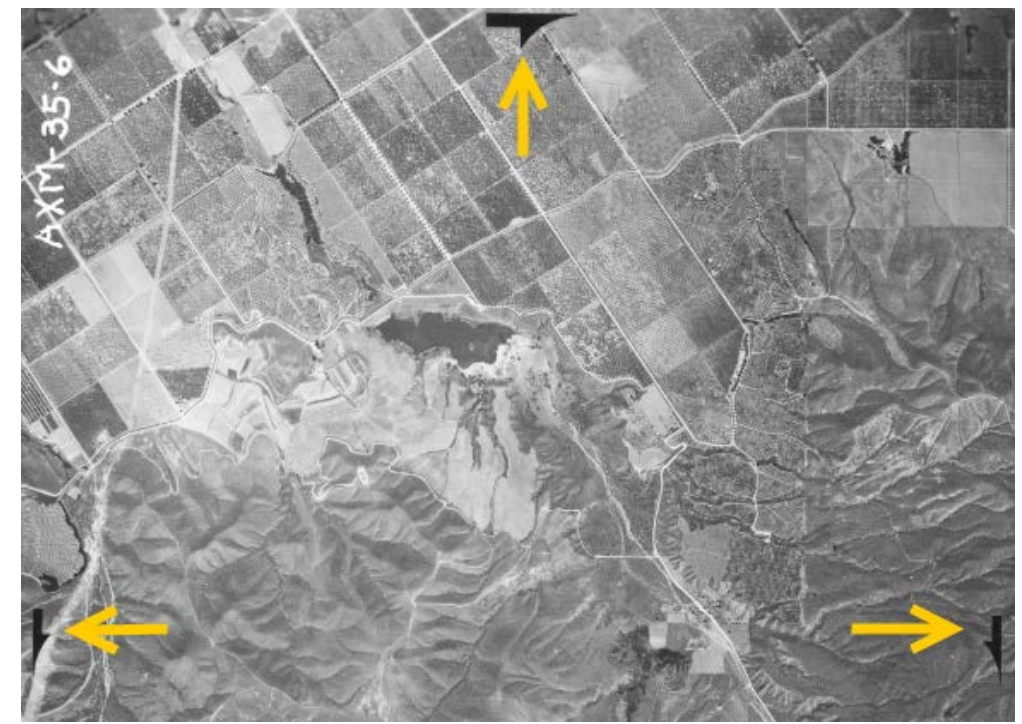

Figure 2-2: Aerial photograph showing the location of three fiducial marks

On each photograph, there are four fiducial marks; they represent the "reference coordinate system for image locations on a photograph" (Wolf \& Dewitt, 2000, p. 58). The intersection of the fiducial marks represents the indicated principle point of the photograph, and their standardized positions in each image allow for calibration of distortion parameters. 
These camera parameters are summarized in a camera calibration report provided by the manufacturer and included with image products. For many historical aerial photography collections, however that information has been lost, and there is limited metadata about the camera used. This is one of the principle sources of uncertainty when working with historical imagery.

\subsubsection{Exterior Orientation Parameters}

A camera's exterior orientation parameters capture the position and orientation of the camera in space. The position is defined by $\mathrm{X}, \mathrm{Y}$ and $\mathrm{Z}$ coordinates in a ground coordinate system with $\mathrm{Z}$ representing the image height above sea level (Jain, Kasturi, \& Schunck, 1995). The orientation is defined by three rotation angles around three axes conventionally named omega, phi, and kappa, they represent the angular rotations of the ground system and the image coordinate system. In a vertical photograph the expectation is that omega and phi would be near zero. As they increase, the tilt of the camera causes the image to skew in the direction of the rotation as shown in Figure 2-3.

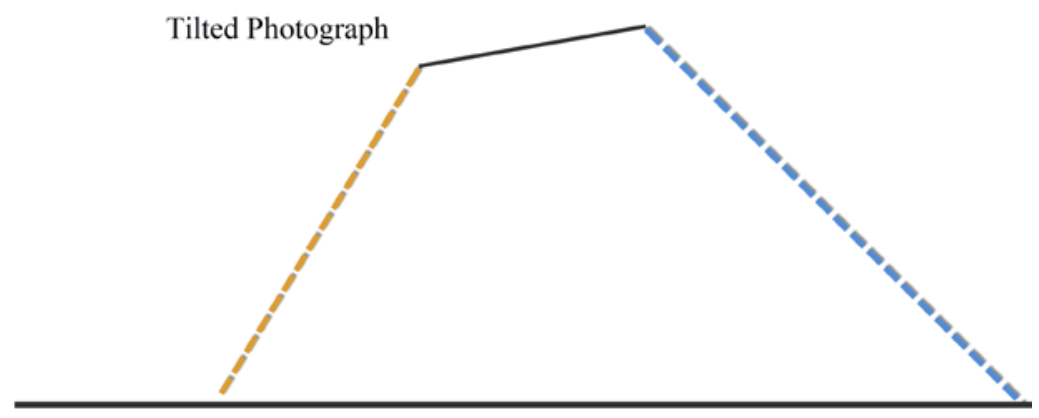

Flat Terrain

Figure 2-3: Tilted photograph showing image skew, adapted from Wolf and Dewitt, 2000, p. 218

Skew causes one side of the image to be closer (orange line) to the ground than the other (blue line) and needs to be corrected. In modern day, GPS positioning and inertial navigation systems provide the ability to track the exterior orientation parameters of a camera in space to a high degree of accuracy allowing for tilt corrections through a process known as rectification.

Historical aerial photographs acquisitions were performed with the camera pointing at nadir to minimize any tilt effects. The average tilt for the Riverside county survey on flat terrain was approximately two degrees, but it was not uncommon to find 5 or 6 degrees of tilt (Monmonier, 2002). Tilt was minimized in the past by making approximate corrections to the resulting calculations (like area) derived from the imagery. It is possible to estimate some exterior orientation parameters of historical surveys, but the estimation contains many uncertainties and assumptions. 


\subsubsection{Relief Displacement}

Relief displacement refers to the distortion caused in an image by the variability of the terrain captured (Wolf \& Dewitt, 2000) as shown in Figure 2-4.

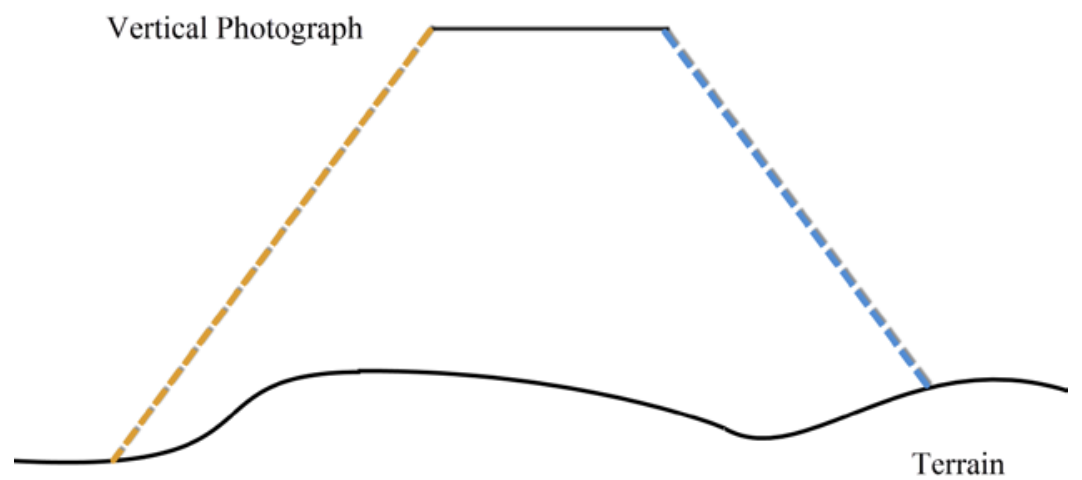

Figure 2-4: Vertical photograph showing relief displacement, adapted from Wolf and Dewitt, 2000, p. 218

Features with a vertical height that is greater than the average surface (blue line) will lean away from the image center and features with a height that is less than the average surface (orange line) will lean towards the image center. The displacement viewed in the image increases with the height change of the object or the distance of the object from the image center. At high altitudes, these distortion effects are mostly observed in significant features like buildings or hilly terrain. Terrain data such as DSMs or DEMs are often publicly available or acquired during image acquisitions, and are used to correct for relief displacement through a process known as orthorectification.

In historical image acquisitions, there are no suitable reference topographic datasets from the appropriate time available. Present day data sources are commonly substituted as a reference, these data contain uncertainty because it is not possible to accurately determine how the terrain has changed since the photographs were first collected.

\subsubsection{Environmental}

Environmental factors at the time of image acquisition can play a huge role in the determination of image quality. These include the time of day, glare, atmospheric conditions like haze, clouds or scene brightness can all play a role (Wolf \& Dewitt, 2000). These effects are managed through adequate project planning to acquire aerial photography in optimal times of day and weather conditions (Wolf \& Dewitt, 2000). When using historical aerial photography, there can often be environmental factors that impact image quality and force an image to be excluded from processing.

\subsubsection{Scanning Film Aerial Imagery}

Aerial photography that was captured on film needs to be scanned into a digital format to be used in computing systems. Scanning converts the analog (continuous surface) into a 
discrete digital raster surface. The quality of the digital image is related to the type of scanner used. Photogrammetric scanners can capture pixels with a high positional accuracy (2-3 microns) and high resolutions up to 5 to 15 microns (Wolf \& Dewitt, 2000). Typical desktop scanners lack the image detail and accuracy to be used in scanning aerial imagery.

Choosing an appropriate scanning resolution can impact the resulting image processing capabilities. Hexagon's Erdas Imagine photogrammetric processing software recommends that orthophoto applications use 15 to 30 micron pixels ( 1500-750 DPI), while applications using aerial triangulation of the imagery use 10 to 15 microns ( 22501500 DPI) (Hexagon, 2016).

The physical scanning process can also introduce small errors into the images that need to be accounted for such as rotation of the resulting scanned image, movement of the principle point (the image center), irregular image sizes from image to image. These small uncertainties can impact processing algorithms and need to be standardized prior to processing.

\subsection{Spatial Referencing and DSM Generation from Aerial Images}

For an aerial image to be useful in analysis it must be referenced to a ground coordinate system. There are many methods of referencing imagery, but the most common are georeferencing and orthorectification.

Georeferencing (also known as rectification) is a 2D transformation that does not require the camera sensor model (interior orientation) or exterior orientation parameters to be known, it uses ground control points to orient images to the ground based on visible features. It corrects an aerial photograph for tilt and image distortion but does not correct for relief displacement, so it is an accurate method of referencing on flat or uniformly sloping terrain but suffers from distortions in areas of high relief displacement (Wolf \& Dewitt, 2000).

Orthorectification uses a $3 \mathrm{D}$ to $2 \mathrm{D}$ transformation and typically requires the camera sensor model and exterior orientation parameters of the camera to be known. Using both known ground control points and elevation data to reference images with a high degree of accuracy. The elevation data is gathered typically from aerial triangulation of satellite remote sensing, terrestrial surveying, aerial lidar or other photogrammetric techniques. Orthorectification corrects for tilt, image distortion, and for relief displacement effects. The resulting images are called orthorectified photos and they are "preferred to rectified photos because of their superior geometric quality” (Wolf \& Dewitt, 2000, p. 217).

It is also possible to extract 3D points to generate a DSM using image stereo matching techniques for height extraction and 3D scene building (Kim \& Muller, 1996). These techniques were developed in the late 1980s and involved "pyramid matching and seed point” techniques.

While orthorectified imagery is the preferred output when referencing historical aerial photography there are some challenges, the interior and exterior orientation parameters may be incomplete or missing and the reference elevation surface may have changed over time. In the case of the Riverside county imagery acquisition the camera calibration report was missing. Most of the internal orientation parameters were unknown or untrusted and the ground features were difficult to determine due to urban 
development since the 1930s. This made most methods of traditional orthorectification ineffective particularly over areas of significant relief displacement.

Overcoming these challenges was part of the research project of Buchwald (2011), whose focus was to develop a method to accurately reference images from the 1938 scanned collection over Riverside county. Initial tests were performed using the georeferencing functionality in ArcGIS 10 using estimations of some internal and external orientation parameters, but the results did not meet the National Map Accuracy Standards particularly in areas of high relief displacement (Buchwald, 2011). To get the required accuracy the project used a method of orthorectification that did not require a rigorous sensor model called a Rational Functional model. The method uses additional ground control to compensate for the lack of interior orientation information and a series of "mathematical functions to represent the relationship between object space and image space” (Buchwald, 2011, p. 9). It delivered better results than simple georeferencing (Rossiter \& Hengl, 2002).

The project was a success as the Rational Functional Model results met the National Map Accuracy Standards; "that 90 percent of all points tested must be within one -fiftieth of an inch on the map" (Buchwald, 2011, p. 37) relative to the ground truth even in many areas of high vertical relief.

After the project was completed the method was not implemented at the CCB because of its dependence on gathering large quantities of high quality ground control. Each image required 20 to 30 manually collected control points that needed to be evenly distributed through the image. The time required to collect the control points along with the specialty skills required to accurately identify and place them exceeded the CCB's availability and skillsets. While this method overcame the requirement of having accurate interior and exterior orientation parameters for processing, it failed to make the process efficient, and resulted in the collection of Riverside county aerial photography to remain ungeoreferenced.

\subsection{Computer Vision and Structure from Motion}

A lot of spatial and temporal information can be extracted from the patterns in overlapping images of the same scene using computer systems (Trucco \& Verri, 1998). The process of extracting, processing, and analyzing information for single or sequences of digital images is known as computer vision. It was conceived in the realm of computer science in the 1950s with the goal of simulating human vision in computing for use with artificial intelligence (Mayer, 2008). As computer systems, robotics and machine learning technology have advanced in the last 15 years so has the technology of computer vision.

The initial focus of the technology was on pattern matching and automation, and less on accuracy (Remondino, Del Pizzo, Kersten, \& Troisi, 2012). This lead to disagreements between traditional photogrammetrists in the 1990s who wanted more emphasis on accuracy and reliability (Remondino, Del Pizzo, Kersten, \& Troisi, 2012) with some indicating that it was not a valid method because it did not use the true calibration parameters of the camera in the solution and was only useful for "Visualization, object-based navigation or other similar purposes" (Remondino, Del Pizzo, Kersten, \& Troisi, 2012, p. 4). Primarily this is because computer vision estimates the internal orientation parameters of the camera and these estimated constants vary from image series to image series even for the same camera. 
In his review of the history of computer vision, Mayer (2008) found examples of how in the early 2000s applications of computer vision technology in photogrammetry started to appear. First, an image panorama was constructed from images without specific knowledge of their scale, ordering or illumination (Brown \& Lowe, 2003). This was followed shortly after by determinations that a series of images could be self-calibrated so long as the perspective of the series were known (Pollefeys, Van Gool, Vergauwen, Verbiest, \& Cornelis, 2004), and finally claimed that the triangulation methods used could deliver a DSM generated from the imagery series that could rival those generated by laser scanners started to appear (Nister, 2004).

In the early 2000s the growth of "web services (e.g. Google Earth, Maps, Bing Maps etc.) increased the demand for orthophotos... and lead to the development of new algorithms and sensors” (Barazzeti, Brumana, Oreni, Preyitali, \& Roncoroni, 2014 , p. 57). This resulted in an eventual hybridization of traditional photogrammetry with computer vision to overcome two chief issues "the automation in the orientation process and the reconstruction process, which results in dense 3D models that in some cases, have a higher resolution that those obtained from laser scanners. Second, the flexibility of the image capturing process, as, due to the substantial technological development of photogrammetric software in recent years it is possible to use any type of image" (GarciaGago, Gonzalez-Aguilera, Gomez-Lahoz, \& San Jose-Alonso, 2014, 6(6), p. 5671). The hybrid approach removes the need for detailed camera input interior or exterior orientation parameters through self-calibration algorithms in the 3D model calculation process of computer vision known as structure from motion (SfM) (Visockiene, Brucas, \& Ragauskas, 2014). These hybrid methods have been adapted into commercial photogrammetric software packages like Photoscan and Pix4D Professional (Visockiene, Brucas, \& Ragauskas, 2014).

An evaluation by Remondino, Del Pizzo, Kersten, \& Troisi (2012) tested different photogrammetric computer vision techniques across a variety of object types to compare their accuracy to traditional photogrammetry. They tested image datasets over cubes, spheres, a lighthouse, a railway and a navona (a piazza). Their conclusion was that the actual final object coordinates were surprisingly accurate (within a scale factor and a check point mean difference of less than $10 \mathrm{~cm}$ ) even though the method does not maintain reliability and repeatability when computing the camera's interior orientation parameters. This was validated by Verhoeven (2014) who also indicated the process fails for individual images or ones that are blurred, noisy or badly exposed.

\subsubsection{Detailed Methodology}

Structure from motion uses algorithms to match features across a series of overlapping images using triangulation; this captures the geometry of the scene, the camera's position (exterior orientation) and an estimate of the camera's calibration (interior orientation) parameters (Verhoeven G. J., 2014). The steps of the process from the source aerial images through to the output orthorectified image as shown in Figure 2-5. 


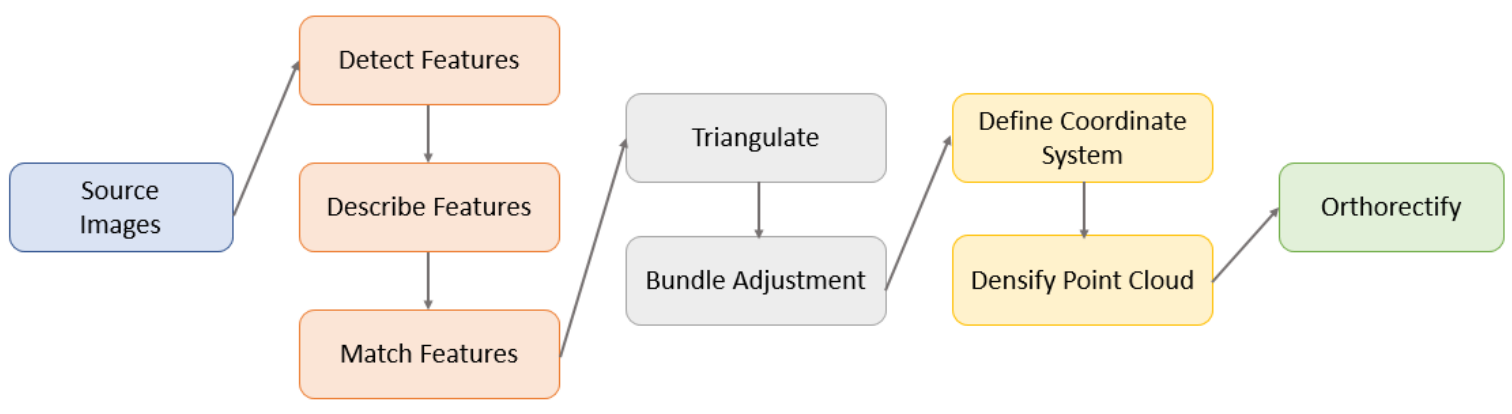

Figure 2-5: Hybrid Computer Vision SfM photogrammetry process, adapted from Verhoeven, Sevara, Karel, Ressel, Doneus and Briese, 2013, p. 43.

First, identification of key image features is required, they are then described in individual images and matched across overlapping images (orange). The matched points are triangulated to create a point cloud and a bundle adjustment is performed to reduce measurement errors (grey). The points are then associated to a ground coordinate system and densified into a point cloud, which is used to orthorectify the images. The details of the steps in the process are expanded on below.

The source images used in the process must be an overlapping sequence, and have at least $60-80 \%$ along track overlap and $20 \%$ sidelap to ensure that the image matching process is effective (Verhoeven, et al., 2013). While results can be obtained from two overlapping images it is preferred that most of the project be overlapped by at least three images for redundancy.

Once input images are acquired feature points must be identified. Success of the triangulation is predicated on objects existing in the imagery that are easy to find reliably and represent key features in the image (Mayer, 2008). Feature points are described in the form of a local vector in the image. The feature description helps match and filter 2D points from different images. Popular feature detectors are Hessian based and are combined with a Scale Invarient Feature Transform (SIFT) algorithm for effectiveness (Verhoeven G. J., 2014).

Once the feature points are extracted and matched their positions are computed in a local coordinate system using triangulation across image pairs as shown in Figure 2-6.

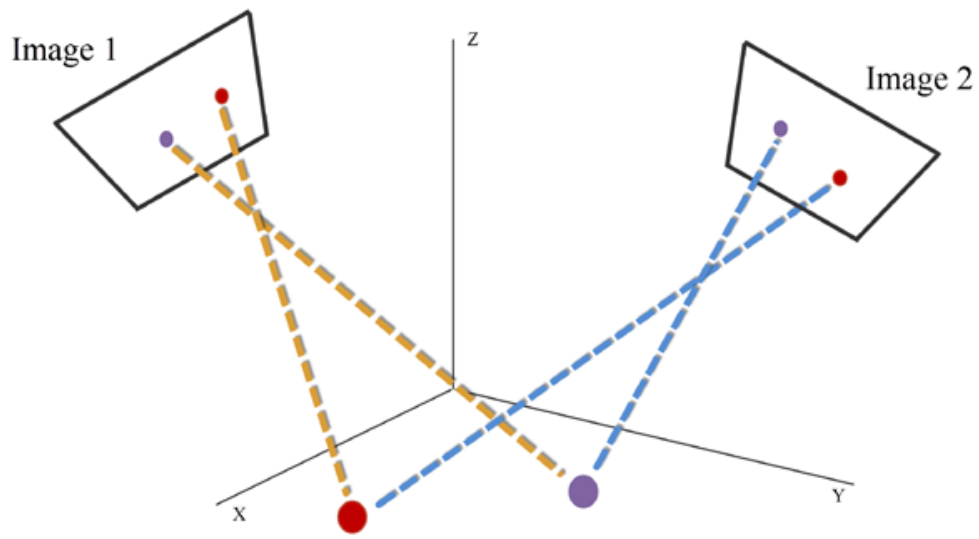

Figure 2-6: Triangulation of 3D points in two aerial images, adapted from Verhoeven, Sevara, Karel, Ressel, Doneus and Briese, 2013, p. 36 
Triangulation orients images together to compute the exterior orientation parameters using tie points between images like the red and purple points in Figure 2-6 (Remondino, Del Pizzo, Kersten, \& Troisi, 2012). The result of the triangulation stage is a 3D point cloud of feature points. As part of this process the interior and exterior orientation parameters of the camera are estimated by self-calibration using the relative orientation of the image pairs (Verhoeven, et al., 2013). The final step once triangulation is complete is to perform a bundle adjustment to iteratively minimize the errors of all images simultaneously between the observed and reprojected image points to compute 3D local coordinates of each point (Szeliski, 2011).

Once the point cloud is generated it is in a local coordinate frame and needs to be related to a ground coordinate system to ensure that it is positioned, rotated and scaled correctly. A minimum of three ground control points are required (Verhoeven G. J., 2014). These control points are applied either during the SfM matching stage as a constraint or outside the process. If they are applied in the SfM process it is typically better as it can "correct for errors such as drift in the recovered camera and point locations” (Verhoeven G. J., 2014, p. 16).

The point cloud generated initially from the method is sparse and needs to be further densified using Multi-View Stereo (MVS) algorithms (Verhoeven G. J., 2014). Densification is necessary to create a DSM of sufficient resolution to be used in the image orthorectification process.

The 3D point cloud is then used to generate a high-resolution DSM of the terrain. By knowing the interior and exterior orientation parameters traditional orthorectification can commence.

\subsubsection{Applications}

Modern day use of the hybrid approach was spurred by the need to handle more images in a semi-autonomous manner. UAV growth in particular forced the development of more robust algorithms due to the use of low cost uncalibrated cameras, the susceptibility of the UAVs to environmental conditions like wind, and inaccurate GPS positioning systems (Eisenbeib, 2009). Despite these challenges it is now however, possible to extract orthorectified imagery, 3D point clouds and meshes from UAV imagery with high automation.

This was demonstrated by researchers at Konkuk University in Korea studying UAVs for use in forest management applications. They used UAVs and this hybrid approach to identify trees and their heights for biomass and carbon emission estimation by creating orthorectified image mosaics and extracting a 3D surface (Ye Seul, et al., 2015). Using a consumer Phantom 3 Professional drone and the Pix4D image processing software they were able to obtain a spatial resolution of $2 \mathrm{~cm}$ at a flying height of approximately 50m. They were then able to use the high-resolution DSM and orthoimages to successfully perform individual tree extraction using segmentation methods. In this case the authors used a common consumer drone without a photogrammetric camera and using the hybrid approach were able to successfully identify and measure trees to under $1 \mathrm{~m}$ of accuracy.

The approach has been applied to traditional aerial photographs as well. The Department of Archaeology at the University of Ghent in Belgium evaluated three case studies of aerial imagery over historical sites in Europe taken from small aircraft 
(Verhoeven, Doneus, Briese, \& Vermeulen, 2012). Each study area had a different terrain complexity, number of available ground control, number of images, and a different acquisition angle for the imagery. In some cases the color images were enhanced to make markings stand out more. The study found that using the Sfm method resulted in a dramatic time savings for processing time and a lower cost (Verhoeven, Doneus, Briese, \& Vermeulen, 2012), and that imagery "that would have been considered unusable” (p. 2066) could still be incorporated in a solution. These images included ones that did not have enough overlap with existing GCPs, ones with a lot of relief displacement without an accompanying DSM to correct them. They also found that large image collections (>200 images) resulted in more being aligned correctly compared with traditional orthorectification methods. They did observe some artifacts from blurry images and insufficient pixel data and the computing power and time for the large acquisitions were significant. While the study did not cover an intense accuracy evaluation the results met the expectations for archeological analysis. The study provided insight that the method can be integrated seamlessly into an existing photogrammetric workflow without a lot of training, and that the investment in technology was easily recovered in time savings. They also found better results using more images to estimate the camera characteristics and increasing overlap to correct issues where possible.

The hybrid computer vision approach has also proven to be robust in unusual environments. It was tested underwater for coral reef mapping by Burns and Delparte (2017) where they used a digital SLR camera to capture overlapping images over the coral systems to define 3D characteristics valuable in research. They compared two commercial software packages (Agisoft Photoscan Pro and Pix4D mapper Pro) and found no significant alignment differences between them for the datasets they had acquired, however they attributed some of that to environmental uncertainty. Overall, they found the technology valuable in evaluating habitat complexity.

\subsubsection{SfM to Historical Imagery}

This approach has been applied to archived aerial photogrammetry at least once, the University of Porto scanned film collections from 1947 and 1958 1:30000 from coverages of Portgual and Spain by the Royal Air Force and the United States Air Force (Goncalves, 2016). Each collection contained 10 to 12 thousand photos, and the SfM method was chosen because previous attempts and conventional implementation of orthorectification were too time consuming and expensive. In this situation, they were also missing the camera calibration certificates and selected 24 photos as a use case. After standardizing the scanned images for rotation and size they ran a similar SfM process using an unspecified commercial software package and successfully extracted an orthorectified mosaic and DSM from the imagery even in mountainous areas. The DSM height differences compared with checkpoints were $-25 \mathrm{~m}$ to +22.7 with a mean of 1.2 and an RMSE of $4.7 \mathrm{~m}$. The image alignment was compared to Open Street Map and considered in general to be good. No specifics were unfortunately provided about issues or areas of improvement, but overall this verified that the method was acceptable for processing large archives in a time efficient and costly manner. 


\subsection{Summary}

Aerial photography has been used for over 100 years as a method of collecting vast amounts of remotely sensed data that can be used in a variety of geographic analysis. The images collected have errors but photogrammetric procedures and technology have steadily improved to correct them and provide a true orthorectified photo and extract terrain information from stereo pairs. In the last 10 years image acquisition has exploded and has driven the development of computer vision based hybrid photogrammetric techniques like SfM to process large volumes of aerial imagery in a semi-automated way. This technology overcomes challenges related to the growing use of low end cameras, camera positioning with environmental disturbances etc. They also provide an opportunity to reexamine our history, and apply this technology to historical imagery in a way that was previously too expensive and time consuming to attempt. 


\section{Chapter 3 - Systems Analysis and Design}

This chapter discusses the system design requirements for extracting image and terrain data products from historical aerial photographs. Sections 3.1 reviews the client's problem that this project resolved. Section 3.2 describes the functional and nonfunctional requirements for this project. Section 3.3 discusses the proposed system design and how it met the requirements set for the project. Section 3.4 summarizes the initial project plan and any deviations or modifications that occurred due to challenges experienced during development.

\subsection{Problem Statement}

Historical aerial images provide valuable geographic information about the past and can be used as a baseline for change management and analysis over time (Morgan, Gergel, Ankerson, Tomscha, \& Sutherland, 2017). To use these images in analysis they need to be scanned, standardized, and processed using photogrammetric techniques that spatially orient them to the world. This is a challenging, slow, and costly process, since supporting metadata about flight acquisition parameters and camera details are often missing for historical images, thus limiting the processing techniques that can be used. This results in many imagery collections being left unprocessed in their raw film state. This project addressed the issue of processing archived aerial imagery efficiently: to spatially orient the images and correct for orientation and distortion errors with minimal human interaction. It also explored extracting valuable terrain surface information through triangulation of overlapping image series using computer vision techniques.

\subsection{Functional and Non-Functional Requirements Analysis}

To successfully plan and complete a project requires gathering detailed system requirements. These requirements set the target expectations for the project and shape the vision of the system that will be built. Requirements are broken into two categories, functional and non-functional. A functional requirement refers to something that the system should do, while a non-functional requirement refers to how the system should work or items associated with the quality of the system. Tables 1 and 2 provide brief descriptions of the functional and non-functional requirements for this project with additional details below. 
Table 1. Functional Requirements

\begin{tabular}{|l|l|}
\hline Requirement & Description \\
Image Standardization Python Script & $\begin{array}{l}\text { The script will provide users with a UI to } \\
\text { select the locations of fiducial marks on an } \\
\text { aerial image. It will then rotate the image } \\
\text { vertically, crop to a standard size and } \\
\text { allow for export to a format and DPI of the } \\
\text { user's choice. }\end{array}$ \\
Orthorectified Imagery Mosaic & $\begin{array}{l}\text { Created from a sample of images around } \\
\text { the City of Riverside. The horizontal } \\
\text { accuracy of the mosaic will meet National } \\
\text { Map Accuracy Standards. }\end{array}$ \\
Digital Surface Model & $\begin{array}{l}\text { Extracted terrain from overlapping image } \\
\text { series. DSM will be clean of obvious } \\
\text { vertical artifacts. }\end{array}$ \\
\hline
\end{tabular}

The primary functional requirements were to create a process to spatially reference the historical aerial imagery and to create a DSM and orthorectified mosaic from the images using aerial triangulation. The acceptance criteria for these requirements were that the orthorectified mosaic needed to meet the United States National Map Accuracy Standards for horizontal accuracy. The 1:20,000 scale of this project resulted in a target horizontal accuracy of approximately 10m horizontally. Precise verification of the DSM was not possible due to the lack of ground truth from 1938. However, the loose DSM requirements were that surfaces deemed stationary should experience under $5 \mathrm{~m}$ of vertical change, and that the integrity of the surface be preserved with few unexplained vertical elevation artifacts.

To meet the acceptance criteria for the information products a script was created that standardized the scanned aerial imagery for orientation and size. The standardization process could be performed manually, but a Python script was developed to reduce processing time and manual effort. Python is used by most GIS organizations, and was genericized to receive different image types, image sizes, and to output customizable image types and DPIs depending on the user's needs. The functional requirements were complemented by non-functional requirements that provided guidance for how the system should work. They are shown in Table 2. 


\section{Table 2. Non-Functional Requirements}

\begin{tabular}{|l|l|}
\hline Requirement & $\begin{array}{l}\text { Description } \\
\text { Reduce Manual Processing Effort }\end{array}$ \\
Coordinate System & $\begin{array}{l}\text { Amage processed. } \\
\text { A workflow that can be easily adapted to } \\
\text { processing hundreds of aerial images in } \\
\text { an archived collection. }\end{array}$ \\
Workflow Procedures for Processing & $\begin{array}{l}\text { The NAD 1983 UTM Zone 11 coordinate } \\
\text { system was used to meet the CCB's } \\
\text { existing conventions }\end{array}$ \\
\hline ESRI Desktop Products & $\begin{array}{l}\text { An instructional guide to perform the } \\
\text { work. }\end{array}$ \\
\hline
\end{tabular}

The major challenges of this project were to find a process that could easily be scaled to hundreds of images without requiring a lot of manual interaction for each image processed. Previous attempts at working with this imagery only worked in the prototype phase on a few images and were unable to scale. For this project that meant reducing the need for ground control (one of the primary time consumers in the first project) and increasing the use of automated algorithms and computing resources to handle the processing load.

\subsection{System Design}

The system was designed to address the client's problem and requirements described above, with the goal of generating a valid DSM and orthorectified mosaic of sample archived aerial imagery over the City of Riverside. The system accommodated two types of users: a basic GIS specialist with some remote sensing experience who would perform the image processing tasks; and a GIS user to interpret the resulting maps and deliverables. The three main software components of the system were a Python script, ArcGIS Pro and Pix4D Professional as illustrated in Figure 3-1. 


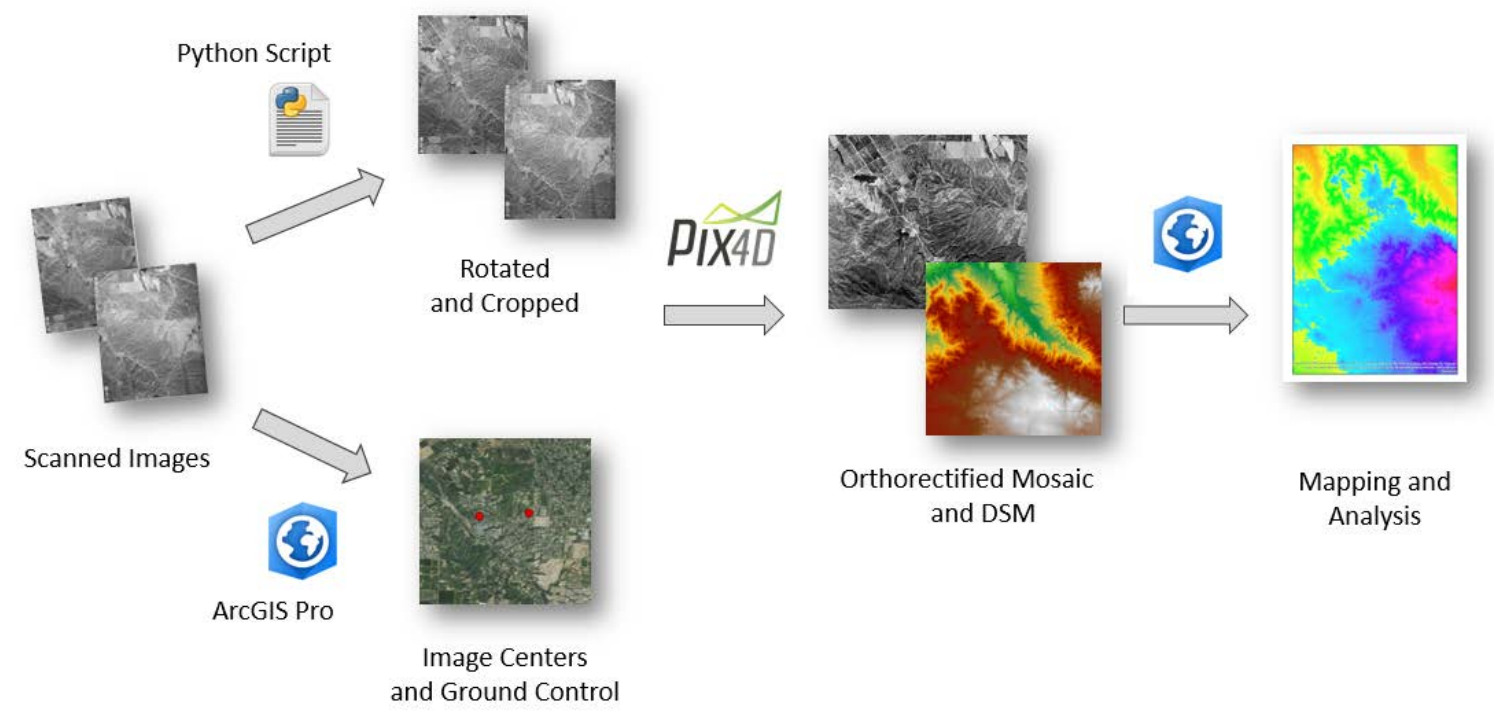

Figure 3-1: System design

Figure 3-1 provides an overview of the workflow of data processing tasks through the system design. The first stage was to prepare the scanned aerial images as they need to be corrected for rotation, image size, and the elimination of erroneous data created during the scanning process. A script was written in Python 2.7 to clean and standardize the input imagery to ensure that each image was the same size, rotation angle, and format. The script allowed the user to input a folder of images; each image was opened in a Graphical User Interface (GUI) and the user could identify the fiducial marks (four or eight) on each photo.

After selecting the marks, the script computed the center of the image, rotated the image to vertical, cropped the image to a standard size from the center point, and exported it. The script used a configuration file for input of settings so that it was generic and could be used by others. The configuration file allowed the user to define the number of fiducial marks on the source images, the target export image width and height, and the export image format. A help document was provided with the script to aid in use.

The second stage - data preparation - included collecting the center of the images (the intersection of the fiducial marks) and correlating it to the corresponding real-world geographic location. Stable ground control points also needed to be identified. These were collected visually using ArcGIS Pro and orthoimage data supplied by the USGS to relate the locations to present day coordinate systems. The vertical position of each image center was estimated as the elevation from the USGS reference DEM plus the approximate flying height for the collection. Both the image centers and the ground control were stored in their own point feature classes in a File Geodatabase and exported to a text file when required for processing.

The data processing of the imagery occurred in Pix4D Pro, which was selected for its robust computer vision SfM photogrammetry algorithm with self-calibration, and its ability to handle large image sizes. The software had many configuration options available to help improve areas with elevation artifacts in the historical image processing. 
The inputs to Pix4D Professional included the standardized scanned aerial imagery, approximate image centers, and the ground control points.

There were multiple steps to processing in Pix4D. First a preliminary point cloud and orthoimage were produced from the image inputs. After processing, they were approximately scaled and positioned in the real world from the input image centers. The processing solution was refined by applying control points in the second stage. The points were included in the solution by tagging them in all visible images and reprocessing to ensure the product met National Mapping Accuracy Standards. To evaluate this, known check points on stable surfaces were found and their coordinates were computed and compared. The third step in refining the solution was to identify manual tie points in areas where there were artifacts in the DSM, and then reprocess the images. The output from Pix4D Pro was an orthorectified image mosaic of the overlapping images and a DSM raster of elevations.

The last step in the system was to perform mapping and analysis with the data products generated from Pix4D Professional in ArcGIS Pro. This included comparing the generated surface against known surfaces for trends and identifying areas of significant change from the past to the present. It also included performing a supplementary georeferencing of the output orthomosaic product to meet the target horizontal accuracy.

The data for this project were stored in a file geodatabase, this included the output imagery products, source image centers, and ground control points. Data input errors were reduced by using domains, standardized naming schemes, strict field lengths, and type restrictions were observed on the database tables.

The data for this project did not need to be hosted and could be stored locally, so there were no specific system communication requirements. There were no specific hardware requirements; this project could be completed by any computer capable of running Pix4D Professional and ArcGIS Pro and both software packages are optimized for a wide variety of common consumer computers. Better computers could complete the work faster but there was no requirement for a maximum automated processing time.

\subsection{Project Plan}

This project faced challenges early in the development cycle because it was a replacement project for one that was cancelled due to onsite data collection access restrictions. Project planning and requirement analysis could not begin until May, 2017. When considering the project management triangle of cost, time and quality, this delay put the most pressure on quality. There was no budget or opportunity to add resources so the cost and schedule were fixed. To complete this project on schedule resulted in added emphasis on the initial planning phase. Detailed conversations with the client were necessary to ensure that there was an accurate scope of work and requirements. From these discussions, a project plan was generated as shown in Table 3, with tasks and an estimate on duration and final schedule shown. 
Table 3. Original Project Plan

\begin{tabular}{|c|c|c|c|c|}
\hline WBS & Phase & $\begin{array}{l}\text { Start } \\
\text { Month }\end{array}$ & $\begin{array}{l}\text { End } \\
\text { Month }\end{array}$ & $\begin{array}{l}\text { Labor } \\
\text { Hours }\end{array}$ \\
\hline 1 & Plan & & & \\
\hline 1.1 & Analyze requirements & May & May & 20 \\
\hline 1.2 & Complete MIP Proposal & May & May & 20 \\
\hline 1.3 & Complete MIP Project Plan & May & May & 20 \\
\hline 2 & Design & & & \\
\hline 2.1 & $\begin{array}{l}\text { Obtain Scanned Images from UC } \\
\text { Riverside Library }\end{array}$ & June & June & 2 \\
\hline 2.2 & Obtain image centers & June & June & 0 \\
\hline 2.3 & Design conceptual data model & June & June & 10 \\
\hline 2.4 & Design physical data model & June & June & 2 \\
\hline 2.5 & Evaluate Data Sources & June & July & 16 \\
\hline 2.7 & $\begin{array}{l}\text { Evaluate Image Processing } \\
\text { Software }\end{array}$ & June & August & 30 \\
\hline 3 & Implementation & & & \\
\hline 3.1 & Prepare database & June & July & 3 \\
\hline 3.2 & $\begin{array}{l}\text { Write image preparation Python } \\
\text { script }\end{array}$ & July & August & 40 \\
\hline 3.3 & Test image preparation script & August & August & 20 \\
\hline 3.4 & Perform data scrubbing & July & August & 8 \\
\hline 3.5 & Collect ground control points & August & August & 10 \\
\hline 3.6 & Preliminary image processing & August & September & 50 \\
\hline 3.7 & Apply ground control in processing & August & September & 10 \\
\hline 3.8 & Reprocessing to cleanup artifacts & September & October & 50 \\
\hline 4 & Analysis & & & \\
\hline 4.1 & Create accuracy assessment & October & October & 15 \\
\hline 4.2 & Create volume change assessment & October & October & 10 \\
\hline 4.3 & Create land cover assessment & October & October & 10 \\
\hline 4.4 & Create other Information Products & October & October & 30 \\
\hline 4.5 & Review and Rework & October & October & 40 \\
\hline 4.6 & Final Implementation & October & October & 40 \\
\hline 5 & Report & & & \\
\hline 5.1 & Draft Final Report & September & October & 120 \\
\hline
\end{tabular}




\begin{tabular}{|c|l|c|c|c|}
\hline 5.2 & Draft MIP Defense & November & November & 40 \\
\hline 5.3 & MIP Defense & November & December & 8 \\
\hline 5.4 & Prepare MIP CD & December & December & 16 \\
\hline 5.5 & Submit to Armacost Library & December & December & 5 \\
\hline
\end{tabular}

In general, the project stayed on schedule, but there were some key issues that impacted the project tasks and schedule. In the original project plan the image photo centers would be obtained from the University of Riverside. However, after initial review they were not accurate enough to be used as image centers for processing. They were approximately $1 \mathrm{~km}$ or more from their true photo centers. Redefining the photo centers and developing a procedure for determining them efficiently became a new project objective. This is a common challenge when working with historical imagery. Some images centers are easy to relate to present-day due to common features like roads, while others are more difficult in rural locations that were developed into urban centers.

While some time had been allocated for testing different processing software for use in completing the project, 30 hours proved insufficient. The original intention was to use Esri's Drone2Map or ArcGIS Pro software, however Drone2Map could not process the image sizes and while ArcGIS Pro 2.0 was able to process the imagery the release available (2.0) did not yet have robust self-calibration functionality for use with historical imagery lacking a camera calibration model. It also had minimal processing options to aid in the removal of elevation artifacts in the generated DSM. Ultimately none of the original proposed software solutions were used to complete the image processing. Pix 4D Professional was subsequently vetted and selected because of its ability to easily handle multiple image sizes, its robust self-calibration computer vision SfM processing technique, and its diversity of processing options needed to optimize and fix many of the artifacts generated in the use of historical imagery. This evaluation process occurred in August, after the intended start time.

The project plan did not adequately address the target coverage of the project area, resulting in the the project area expanded multiple times. The expansions occurred to include a wider range of terrain types, elevations, and features of interest. This included obtaining more coverage over Lake Mathews, which was just being constructed in 1938. Each expansion of the project area required acquiring new images from the library, cleaning them, finding the image centers, finding ground control and including them in the processed solution which was more time consuming than initially thought.

Lastly, at the start of the project a landcover assessment was one of the information products and would be created to illustrate how the use of the terrain had changed from the 1930s to present day to show the progression of urban development in the area. Due to some of the processing challenges this could not fit in the timeline of the project, and was cut to ensure the completion and quality of the remaining products.

The project was successful because of regular communication with the client to ensure that the project was on schedule and by adjusting the scope when necessary to successfully complete the project. Initially the scope was too optimistic for the compressed timeline which was compounded by the processing challenges mid project. 


\subsection{Summary}

Planning and system design are critical components of any project. Good planning sets and communicates project expectations to all parties involved and reduces the risk of project failure. Investing time in documentation and system design at the start of a project allows the project team to tailor a solution appropriate for a client's problem and organization within the target time and budget. The solution developed for this project solved the client's primary concern of spending too much manual effort processing each image. It also met the functional requirements of generating an orthoimage mosaic and a digital surface model. 


\section{Chapter 4 - Database Design}

This chapter reviews the database design and implementation concepts as well as the data required to complete this project. It was not a client requirement to create a complete database management system, so the database design described in these sections was made to fit only the requirements of the project. It could, however be used as a framework for further expansion in other similar projects.

Section 4.1 explains the conceptual database design for the project's attribute and spatial data. Section 4.2 reviews the implementation of the design with a logical data model. Section 4.3 reviews the data sources for the project, and Section 4.4 the methods that were employed to prepare and clean the data.

\subsection{Conceptual Data Model}

This project did not require a complex database design because the primary inputs and outputs of the system were raster images. There was limited supporting information available, and only a small subsection of it was required in processing. The conceptual model was divided into two sections: the first defined the structure of project metadata; the second showed the visualization of image storage through the project lifecycle.

The project information was broken down into the following entities: Project Area, Reference Points, Flights, Flight Lines, Image Centers and the Camera. A conceptual model was created to show the relationship between these entities in the database as illustrated in Figure 4-1.

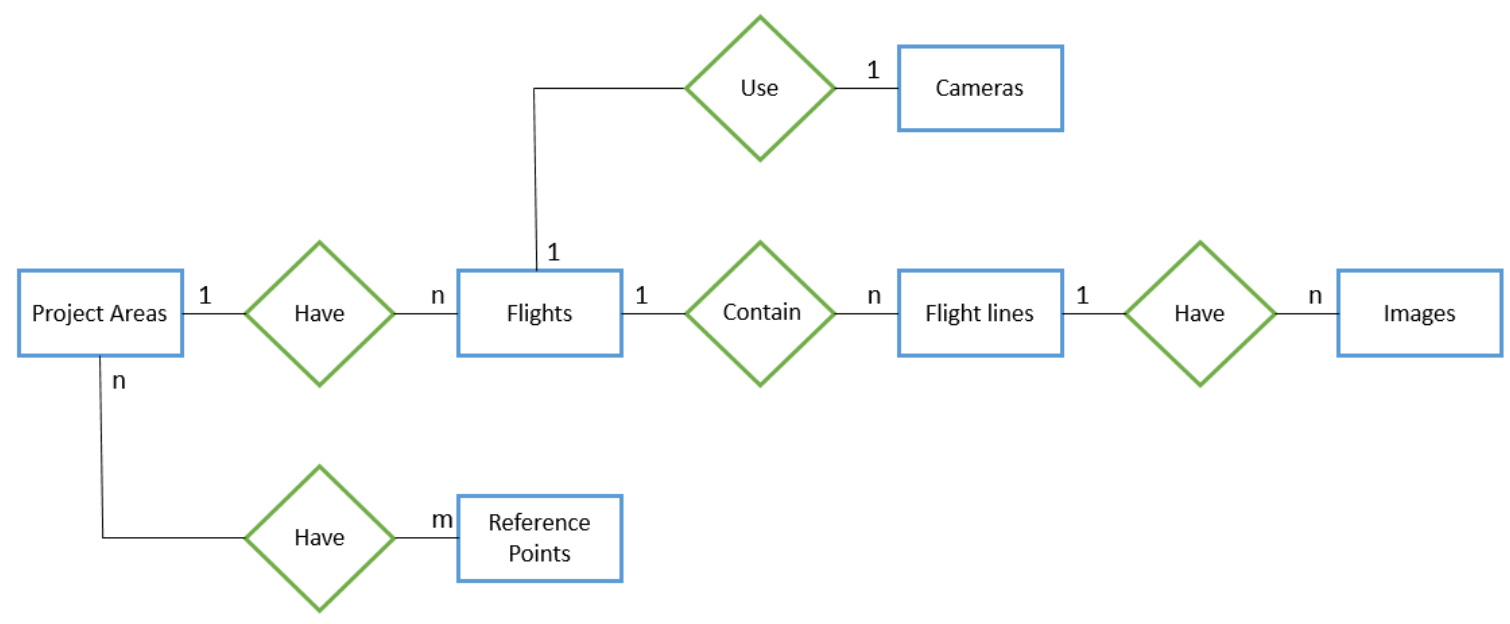

Figure 4-1: Conceptual data model

The highest level of the conceptual model was the project area, and within the project area were the reference points and flight information used in processing. The relationship between the project area the reference points was many to many because even though the reference points were captured for this project they could be applicable to other projects that overlap the same area. The Riverside image collection used in processing was only one of many historical image collections managed by the CCB. 
The boundary of the project area contained all the flights flown, a single project could have many flights. A flight had many flight lines. Each flight used only a single camera at a time to capture imagery. At the lowest level of the conceptual model were the image centers; each flight line had many images associated with it. These entities represented the conceptual model of all the associated project information.

The images used for this project underwent a transformation from their initial raw scanned state to final image product. The conceptual model of how these images were stored through the process is shown in Figure 4-2.

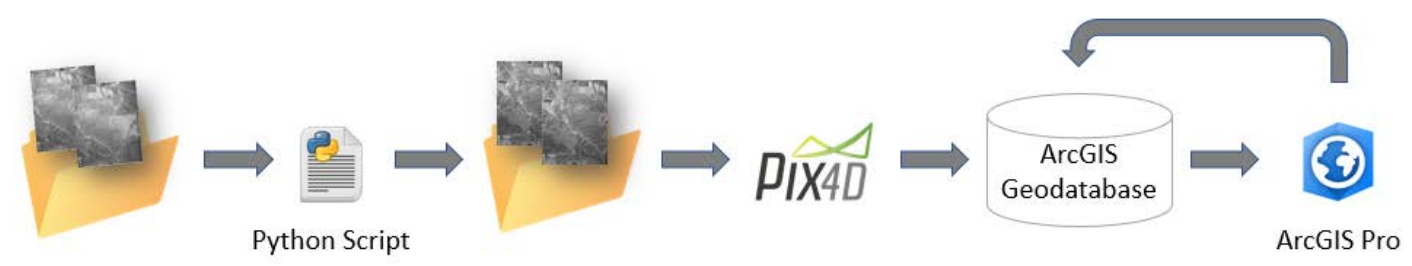

Figure 4-2: Conceptual data model of image storage

The images that were received from the University of California, Riverside library were stored in a folder on disk, as the Pix4D Professional could not import them directly from a file geodatabase. The Python script that standardized them for size and orientation imported them from their initial folder, performed the transformation, and exported the corrected images to an output folder. Pix4D then imported the corrected images and generated a single orthorectified image mosaic of all input imagery and a DSM. These two rasters were stored in an ArcGIS Geodatabase. Subsequent image products generated in analysis in ArcGIS Pro were also stored in the same ArcGIS Geodatabase.

\subsection{Logical Data Model}

The logical data model provides a detailed examination of the ArcGIS Geodatabase contents for this project. Designing a complete data management system was not a client requirement for this project, so the database was primarily a sample of the relationships and supporting information used only in processing. The intention was to create a schema that could be used for testing, and considered as a starting template that could be adapted for use in other projects.

The entities defined in the conceptual model still applied when the logical model was constructed; however, some entities did not have many supporting attributes and were primarily used only as a reference geometry. This is discussed in more detail when examining the individual entities. Figure 4-3 shows the logical data model of the database. 


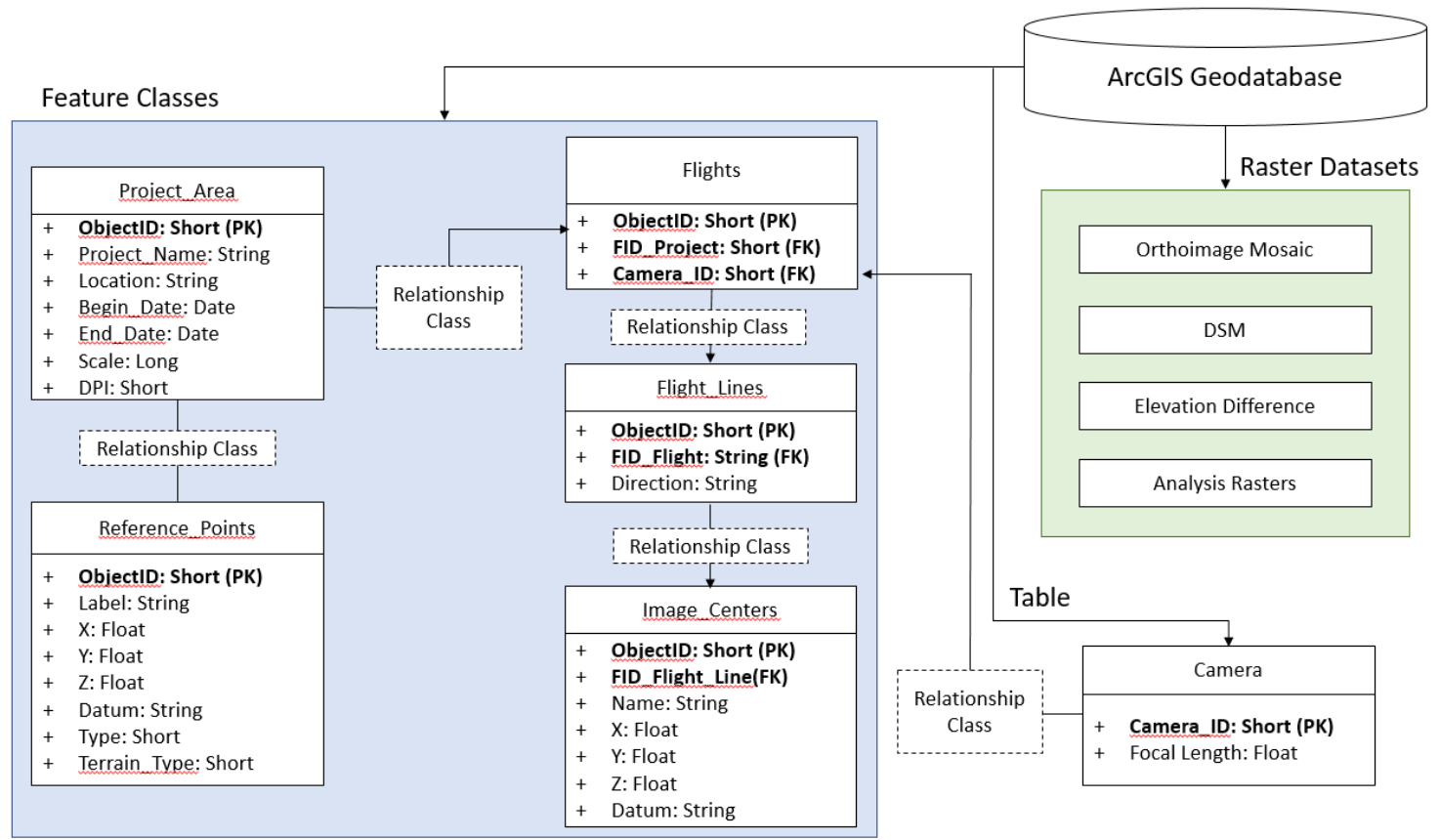

Figure 4-3: Logical data model

There were four principle components to the logical data model: the ArcGIS File Geodatabase that stored the project information; the five feature classes which contained geometry and reference information about the project; the three raster datasets which were created as output from Pix 4D Professional or ArcGIS Pro; and the Camera table which contained the only camera detail known for this project. Each feature class contained attributes that supported the image processing effort or reference information about the project. All feature and raster data were stored in the NAD 1983, UTM Zone $11 \mathrm{~N}$ coordinate system. The relationships between the entities as defined in the conceptual model were maintained using geodatabase relationship classes. In the many to many case of Project_Areas to Reference_Points, this resulted in an additional table automatically being created in the geodatabase to maintain the relationship between the primary keys from the two tables. The relationship classes were established using the simple type, which allowed for reference points to be added or removed without impacting the Project_Areas feature class.

The two principle feature classes used in processing were the Reference_Points and Image_Centers point feature classes. The Reference_Points feature class was used to store two types of reference points: ground control points which positioned and scaled the images during processing and independent check points used to provide a relative measure of accuracy. These points were identified visually by cross-referencing the source images with current reference imagery. Each reference point had a label to indicate its name (per Pix4D naming standards) and coordinates in the processing projection. The Type attribute was used to indicate whether it was a ground control or check point; this was implemented as a geodatabase domain so that only those two selections were available. The Terrain attribute was used to indicate the type of terrain the point was captured over and it was also implemented as a geodatabase domain type. Values for it included: farmland, road, mountain etc. The Image_Centers point feature 
class was used to store the location of each image center. The centers were identified visually from reference imagery. Each image had a Name attribute which corresponded to the file name of the photo and its.

The other feature classes of Project_Areas, Flights, and Flight Lines as well as the Camera table were all used to hold supporting attribute information about the project. Project_Areas was a polygon feature class that held the extent of the area for reporting along with some metadata about the project. Flights was a polygon feature class that held an estimated extent of each flight. Flight_Lines was a polyline feature class that held the approximate trajectory of each flight line and the Cameras table held the only detail about the camera known for this project, its focal length. The feature classes and table were not actively used in processing but completed the entity relationship and were used in reporting for the project. In other aerial processing projects of a similar type this schema could be expanded with additional fields. For example, the Image_Centers feature class could be expanded to contain fields associated with the approximate rotation of each image and then be considered a frame table. The Camera table could also be expanded to include more attributes associated with the interior orientation of the camera. Since these details were not available for this project and creating a complete database management system was not a client requirement they were omitted.

The final orthorectified images, DSMs, and elevation analysis rasters were stored in the geodatabase as raster datasets. There was no requirement to tile the output into specifically sized tiles, so the large area raster datasets were a sufficient method of storage.

\subsection{Data Sources}

This project required a few input data sources including: the original scanned aerial images, approximate image centers of each photo, and reference orthomosaics and DEMs for a relative ground truth comparison.

The source black and white aerial images used for this project were provided by the library of the University of California, Riverside from collection AXM-1938. A sample of 28 overlapping images from five flight lines were used. The original 7.25 x 9.25-inch film photographs were already scanned to 1200 dpi and provided as TIFs.

There was no calibration report available for the camera so minimal information about the manufacturer or interior orientation parameters of the camera were available. The only reference information available about the original acquisition flights was found on the UC Santa Barbara website (University of California, 2012). The site indicated that it was flown at a scale of 1:20,000, with a flying height of 13,750 feet, $60 \%$ overlap of photos along the same flight line and $20 \%$ sidelap between adjacent flight lines, and that the camera's focal length was 8.25 inches.

The initial image centers were provided by the library of the University of California, Riverside in kmz format in geographic coordinates. They were initially converted into an ArcGIS feature class stored with the belief that each image center was within approximately $50-100 \mathrm{~m}$ of the true image center as a reference. This assumption proved to be false and the image centers were recollected for processing. The original values were invaluable though as a preliminary photo index and indicator of project extent 
The reference DEM used for this project was acquired from the USGS (Elevation, 2017) as part of their ongoing 3D Elevation campaign to provide elevation data across the United States. The resolution of the DEM was $1 / 3$ arc second (approximately $10 \mathrm{~m}$ ). The DEM was provided in geographic coordinates as GCS North American Datum 1983 (NAD83). The RMS of the DEM was not listed as part of the associated metadata, because the USGS DEMs are constantly being updated and are a combination of multiple data sources (Elevation, 2017). A study by FEMA estimated that in general the 1/3 arc second NED DEM had a vertical RMS of approximately 1.64m (FEMA, 2007). Despite being a 10 year old assessment, this vertical RMS was assumed to be correct for this project, as a more current assessment could not be found. Due to the historical nature of this project and uncertainty in the terrain, a more precise or current value was not required.

Reference color orthophotos were also obtained from the USGS High Resolution site (USGS - Longterm Archive, 2015) which had a resolution of $0.3 \mathrm{~m}$ and a recorded horizontal RMS of $0.225 \mathrm{~m}$ as listed in their accompanying metadata. They were acquired in 2014 by the USGS and provided in GeoTIFF format with a spatial reference of NAD83 UTM Zone 11 North.

\subsection{Data Collection Methods}

Most of the required project data were provided by the University of California, Riverside or acquired from the USGS, so data collection for this project was minimal. The initial image centers that were provided were inaccurate, so they were recollected into a point feature class. The center of each photo was determined by the intersection of the vertical fiducial marks with the horizontal fiducial marks and then correlated to the reference imagery. Additionally, ground control and check points needed to be identified and collected from the reference imagery. The complete methodology for collecting both of these data types is summarized in Section 5, as the workflow was an integral part of the data processing for this project.

\subsection{Data Scrubbing and Loading}

The University of California, Riverside provided the 1200 dpi scans of the aerial photos. The overall scan quality was good, but the scanned images had not been cropped to a specific image size to ensure the image center was constant and the orientation was vertical and uniform for each image. To be used in processing they needed to be standardized to be uniform; this was completed using a Python script. The development and methodology of this script is discussed in Section 5.

Additional standardization of the input data was required to ensure that it was reprojected to the UTM Zone 11N coordinate system and NAD83 datum. This was performed using the tools in ArcGIS Pro.

\subsection{Summary}

Initial project planning created a conceptual model design of a database that was extended in implementation into a logical model. While there were no requirements to completely define a data management system for the client, the database schema that 
resulted from the logical model could be extended for use in other archived imagery processing projects.

The information and data received from University of California, Riverside library required some cleaning but was successfully able to be used on this project. The reference data used for this project primarily came from publicly available sources that required little scrubbing or maintenance. When possible existing tools were used to automate the data clean-up procedure. Proper data maintenance and management formed the foundation for this GIS project. 


\section{Chapter 5 - Implementation}

This chapter describes the project implementation details, including the methods and software used to complete the processing and analysis of this project. Once the data were acquired and the database design completed, the processing workflow was designed so that raw scanned aerial images could be processed into an orthorectified image mosaic and DSM. The required stages to complete this process are described in this chapter.

Section 5.1 reviews the image standardization process, which corrects the scanned aerial images for orientation and size. Section 5.2 reviews how to determine the coordinates of the center of each image, and use them to create the necessary project overview feature classes. Section 5.3 reviews strategies for capturing the required reference control points. Section 5.4 reviews the steps of the image processing workflow in Pix 4D Professional, and Section 5.5 discusses the generation of analysis products from the resulting processed imagery and digital surface.

\subsection{Image Standardization}

Each TIFF aerial image obtained from the University of California, Riverside library was sized and oriented differently due to the manual scanning process employed. This resulted in an image center that varied from image to image. The Pix 4D Professional's self-calibration method computed one standard correction per project based on the assumption that all the input images had a standard format. The small misalignments in the scanned image collection for image center, size, and rotation would have affected the final product quality without correction. To standardize the images a Python script was developed; the steps of the script are shown in Figure 5-1.

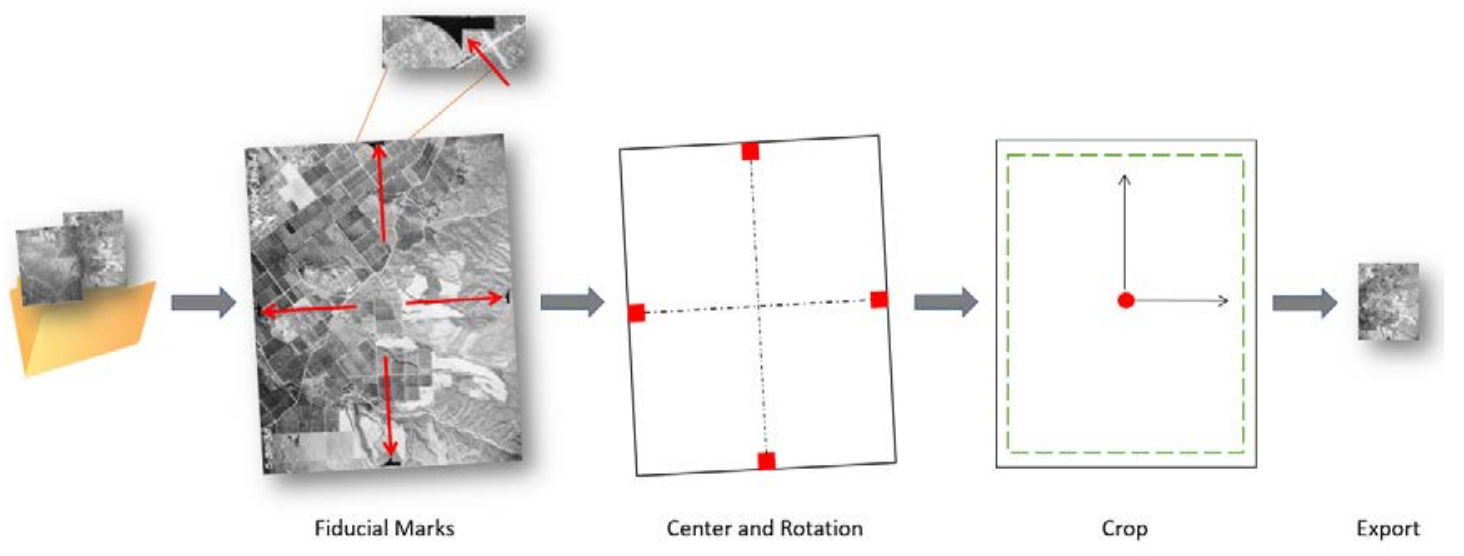

Figure 5-1: Python script image standardization process

The Python script iterated through a folder of input images to capture the image coordinates of the fiducial marks, and use those positions to compute the image center and required rotation angle to vertically orient them. Once rotated, the tool exported a cropped version of the final image to a new file, along with a separate smaller image to aid in correlating the image center to ground reference imagery. The input parameters: 
image type, export type, export DPI and final image size were stored in a separate configuration file and loaded on startup.

The first step in the script was to capture the image coordinates of the fiducial marks on each image. It was not within the scope of the project to design an automated tool to determine the location of the fiducial marks, so a simple UI was created to load each image and allow the user to manually select each fiducial mark. The script used the Tkinter Python module to generate the GUI and load the imagery as shown in Figure 5-2.

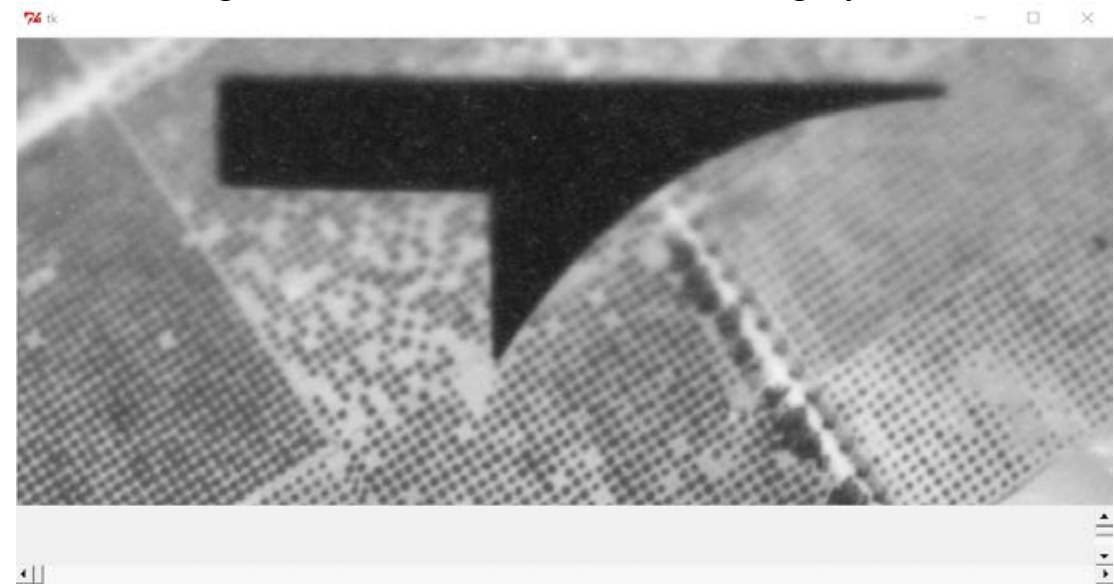

\section{Figure 5-2: Image standardization GUI}

Using the GUI, users select the intersection of the horizontal and vertical lines of the fiducial mark to capture the coordinate. After confirming the input, they use the scroll bars to navigate to the next fiducial mark until each one for the image are captured. The script recognizes the mouse click events on the screen, and uses them to compute a coordinate from the top left location of the GUI image window relative to the top left location of the image from when it was first loaded. Adding the two together calculates the final coordinate for each fiducial mark. Once the target number of fiducial marks were captured the GUI closed automatically.

In the second step, the image center and rotation angle are computed and the image rotated so that the fiducial marks align horizontally and vertically around the image center. The image is reloaded by the PIL Python image library module. The image center is computed by averaging the coordinates of the four fiducial marks. The required rotation angle is computed by using arc tangent and the horizontal and vertical differences between the upper fiducial mark and the image center. The image is rotated, which required resampling. The nearest neighbor algorithm is used as the interpolation method to perform the resampling.

In the third step of the script the image is cropped to the final target dimensions supplied in the configuration file. The values are determined by examining the edges of the images and determining the maximum image dimensions while still removing any excess caused by the initial rotation or scanning method. A sample of an original image and the same one after standardization are shown in Figure 5-3. 

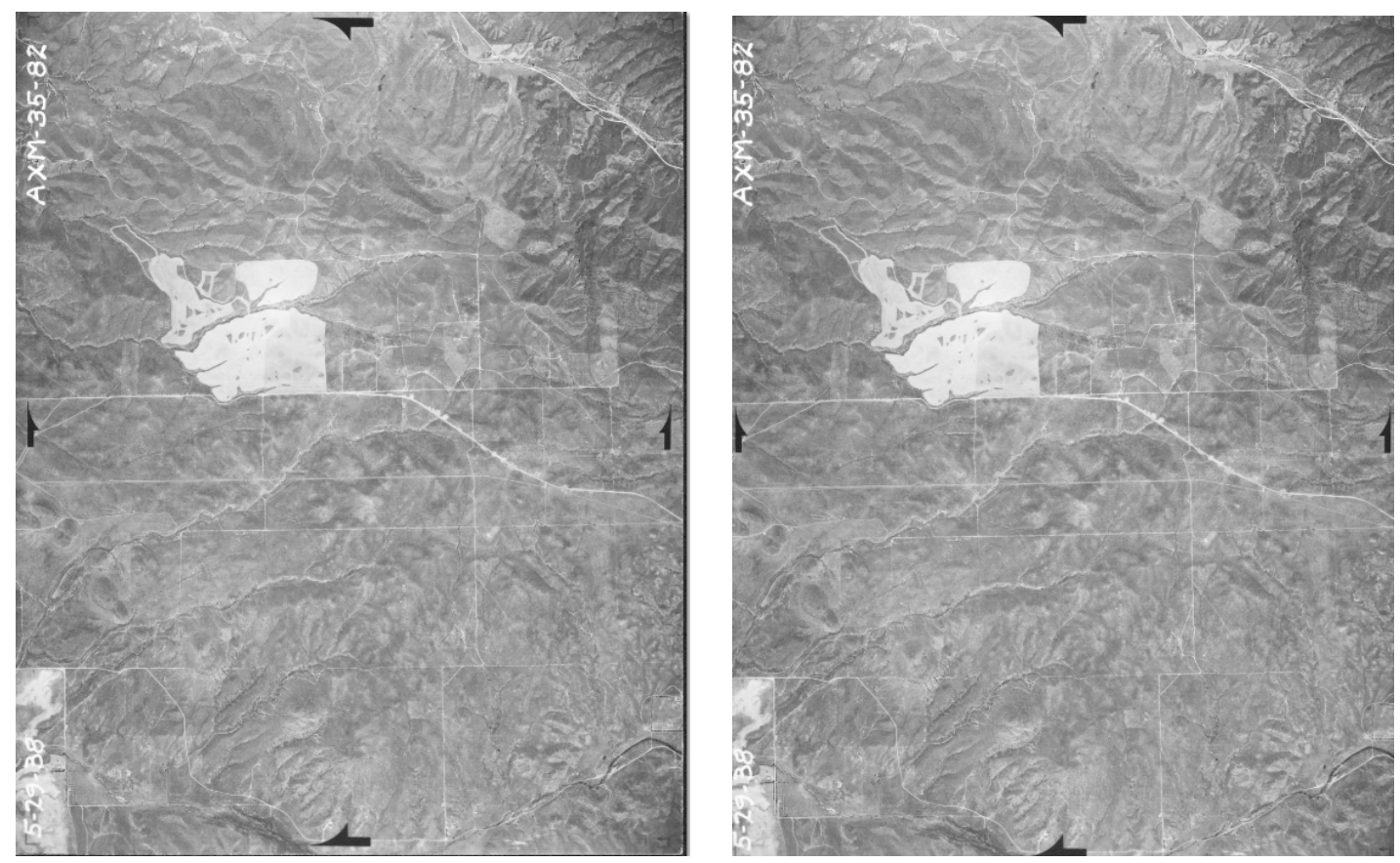

Figure 5-3: Original and standardized photo

The excess in the image was represented by the black strip as shown in Figure 5-3. Cropping out only the fewest number of pixels helped minimize the reduction in image overlap between adjacent images in a flight line or between flight lines, because the Pix4D processing method was dependent on image overlap. In the final step of the script, the standardized image is exported to the specified output format and DPI defined in the configuration file.

\subsection{Image Center Coordinates}

Determining the spatial coordinates of the image centers was challenging because the terrain over Riverside County has changed since 1938 when most of the landscape was farmland, with some development. Currently much of the land is developed. The primary method of determining the image center's spatial coordinates was to visually correlate common features between the image and the USGS basemap, shown in Figure 5-4 below. 


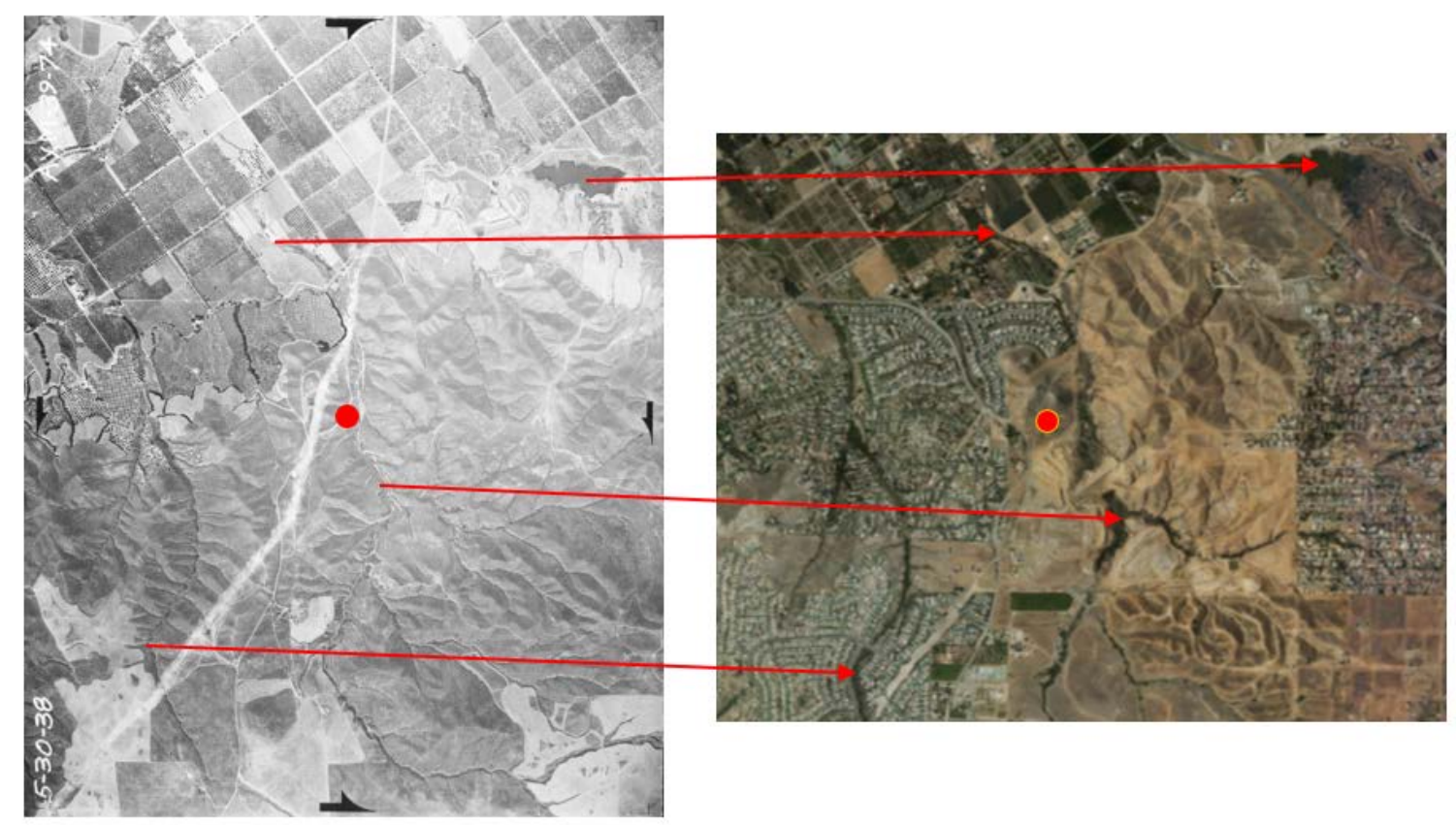

Figure 5-4: Correlating common features of a historical photo to present day

As shown it is easy to identify common features, and determine an approximate horizontal position of the image center. But for many photos it was too difficult or time consuming to find the image center in the basemap. To solve this problem a preliminary Pix4D Professional project was created to roughly position a coarse orthomosaic of the historical photos using the minimum required inputs. Pix4D Professional does not require that all the aerial imagery have known coordinates to complete a processing session, only three images in the collection need coordinates. The generated mosaic was not accurate ( $200 \mathrm{~m})$ but it did merge the historical images into a seamless mosaic which could be correlated to the reference image mosaic. The Pix 4D processing workflow is summarized in Section 5.4.1.

The image centers were captured into the Image_Centers feature class using ArcGIS Pro. The horizontal coordinates in NAD83 UTM Zone 11 were computed using the Add Geometry Attributes tool. The elevation of the image center was computed by intersecting the image centers with the USGS reference surface using the Add Surface Information tool and adding the approximate altitude of the flights.

\subsection{Reference Control Points}

Reference points were established to scale and orient the final data products to the target spatial reference system. Check points were also established to provide an approximate accuracy assessment of the adjustment. The coordinates of the reference points were established in NAD 83 UTM Zone 11, and identified from the USGS reference imagery. While they were used as control, their horizontal and vertical positions contained uncertainty because they were established using present day reference data as opposed to those from 1938. It was impossible to know the uncertainty of each reference point's spatial position from now to then. To mitigate this issue the reference 
points were selected on surfaces considered stable over time. A minimum of three control points were required for processing. Ground control points were not required for each photo. In selecting ground control points preference was given to areas where there was $300 \%$ or more image overlap. Some examples of feature types that were chosen as ground control or check points are shown in Figure 5-5.
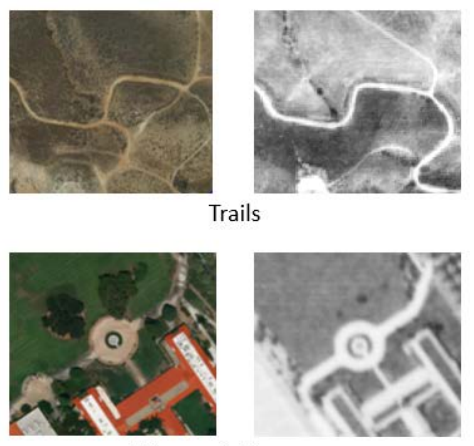

Manmade Features

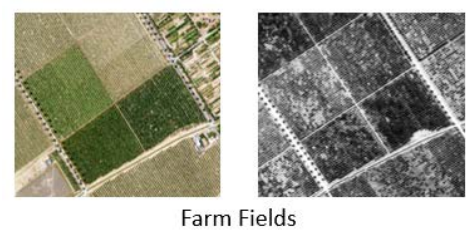

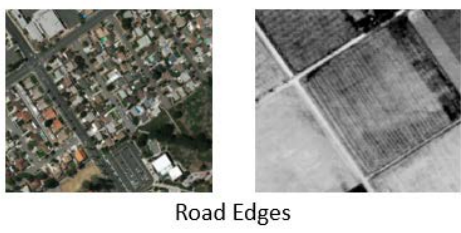
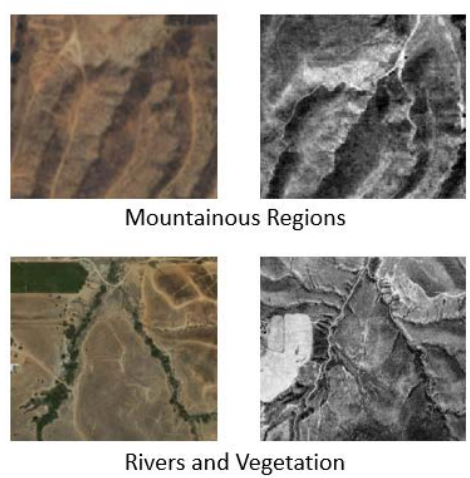

Figure 5-5: Reference features

In open terrain, a trail with a sharp bend could serve as a 3D control point due to the path being clearly visible and the elevation consistent. In urban areas, stable manmade features like the courtyard of California Baptist University (Figure 5-5, left column, middle pair) could be used, or the intersections of secondary residential roads. Primary roads were not used as they were often completely rebuilt and redesigned. In farmland, the intersections of farming quarter sections in orange groves were reliable horizontal and vertical control points. Mountainous regions and areas of consistent vegetation or hydrology provided valuable horizontal check points but inconsistent vertical results.

The reference points were captured in ArcGIS Pro and added to the Reference_Points feature class with an attribute for their type and the terrain they were found on. The initial control and check points that were captured for the project are shown below in Figure 5-6. 


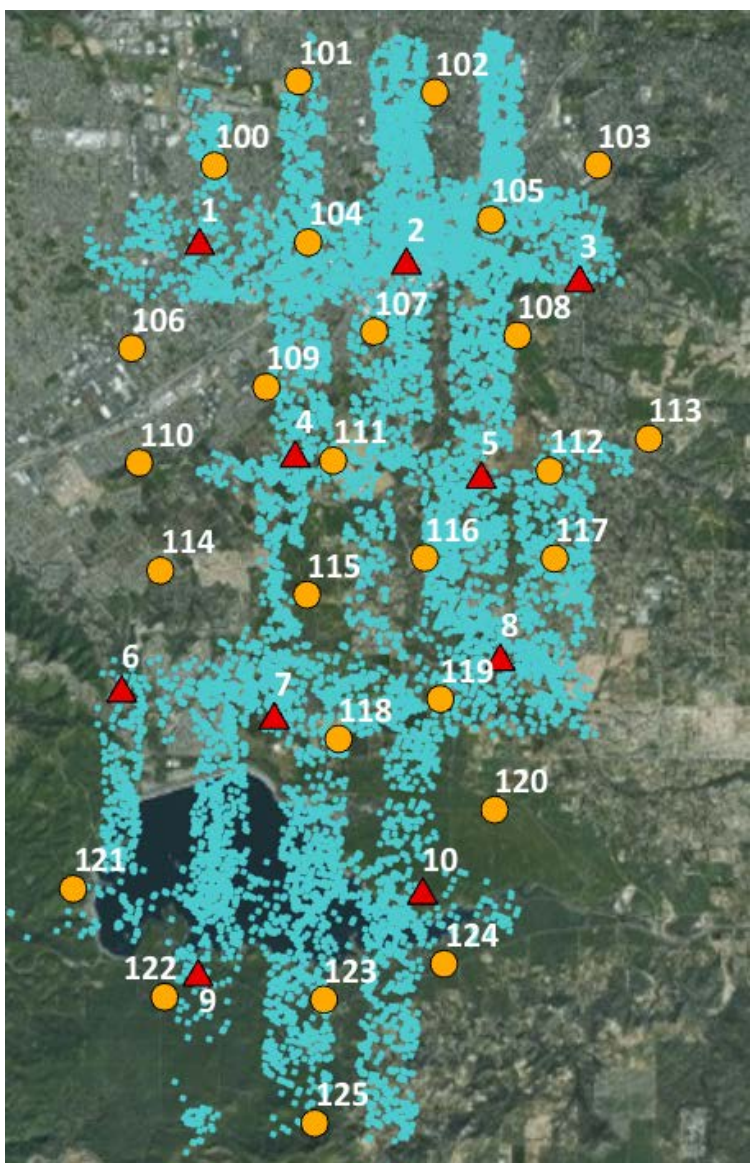

Figure 5-6: Ground and check points

More reference points were captured than were required for additional redundancy in testing and evaluation. They were used in different combinations depending on the testing scenario as discussed in Section 6.

\subsection{Generate Orthophoto and DSM}

Pix 4D Professional was selected as the processing software for this project due to its ability to process large numbers of images without any knowledge of the camera's calibration parameters. Pix 4D was also a mature image processing software package, and had advanced settings that could be changed to fix issues with the imagery and improve product quality. The Professional option accommodated larger image sizes (>50 MB), which was important for this project as the 1200 DPI images were over $70 \mathrm{MB}$ each. Esri’s Drone2Map 2.0 was considered, but could not process images larger than approximately 50MB. ArcGIS Pro Version 2.0 was also considered, but it was released after the project lifecycle had already commenced. The processing workflow in Pix 4D Professional was a multi-phase process; the steps are shown in Figure 5-7. 


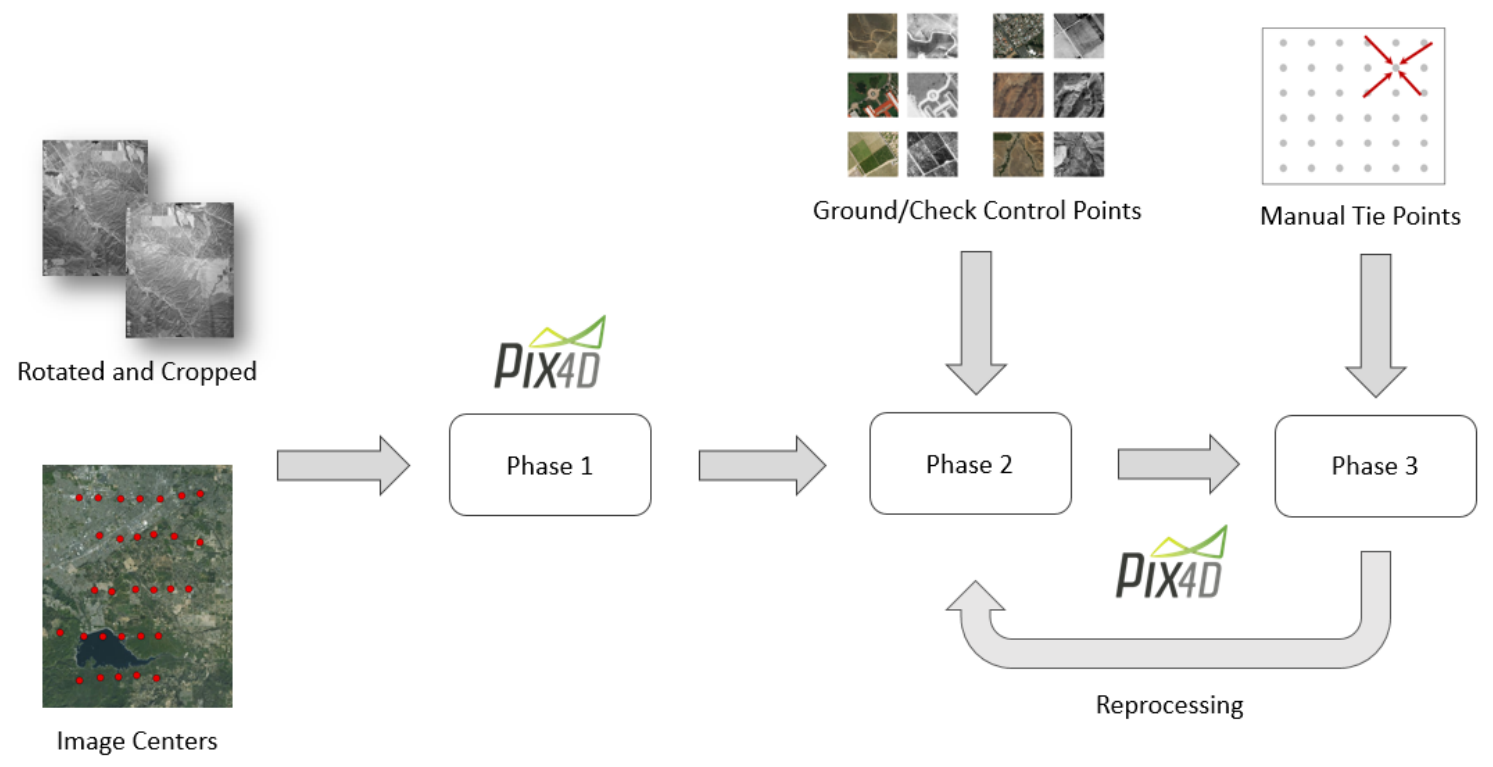

Figure 5-7: Pix 4D processing workflow

In the Phase 1 of processing the Pix 4D project was created, the images imported and the first processing session was run to compute tie points between the images and preliminary image positions based on the input image centers. In Phase 2, the results of Phase 1 were evaluated and ground control and check points were added to the project to scale and orient the solution. In Phase 3, manual tie points were created to fix areas where elevation artifacts were present in the DSM. After Phase 3 the solution was refined through iterations of reprocessing. The following sections discuss each phase in more detail.

\subsubsection{Phase 1 - Initial Processing}

After the Pix 4D project was created the aerial images were loaded from a local folder, and their image center coordinates imported from a text file. The processing coordinate system of the project was set to NAD 83 UTM Zone 11N. The geolocation accuracy of the points was set to low (50m horizontally and $100 \mathrm{~m}$ vertically) to account for the uncertainty in the image center positions. By default, Pix 4D was configured to calculate tie points in areas that had three or more overlapping photos. The historical collection of aerial images used for this project, however, only had small areas of three image overlap so the setting had to be modified to process on two or more overlapping images. This introduced some uncertainty but was required to obtain full coverage over the project area. Additionally, the initial setting to create a DSM was set to process at a grid size of $10 \mathrm{~cm}$ which was too fine a resolution for the project so it was increased to $5 \mathrm{~m}$ for early processing phases which lowered the required processing time.

After processing was completed, a preliminary processing report, orthomosaic and DSM of the area were created. The results of the preliminary processing report were used as a method to verify the integrity of the input images and image centers. The report identified whether: 
- All the images had been incorporated into the solution. Missing images might have invalid or missing image coordinates, or could not be matched.

- Each image had enough keypoints $(>10,000)$ identified. Keypoints were an important indicator of visual differentiation in the image.

- Enough matches (> 1000) were found between images. The more matches the better the calibration results.

To correct any issues required reviewing the image center coordinates, the standardization process or the image scans to correct any issues with the source data. The results of the processing reports for this project will be discussed in more detail in Section 6 and the final processing report can be found in Appendix B. The data products from Phase 1 were good for preliminary visualization only, and could be used to help define the image centers in Section 5.2. To improve the solution ground control points were added to the project in Phase 2.

\subsubsection{Phase 2 - Intermediate Processing}

In Phase 2 ground and check points were imported to control the scale, orientation and position of the solution, and to provide checks on the processing quality. These reference points had known positions, and were selected over areas assumed to be stable with the knowledge that uncertainty was unavoidable.

Their input coordinate system was set as NAD 83 UTM Zone 11N, and their type set as a $3 \mathrm{D}$ ground control point or as a check point depending on their target use. Their accuracy was set to low (5m horizontally and vertically) to account for the uncertainty in their positions. Once loaded into Pix4D they were tagged in every image they were observed in using the rayCloud viewer. The process is shown in Figure 5-8 below.
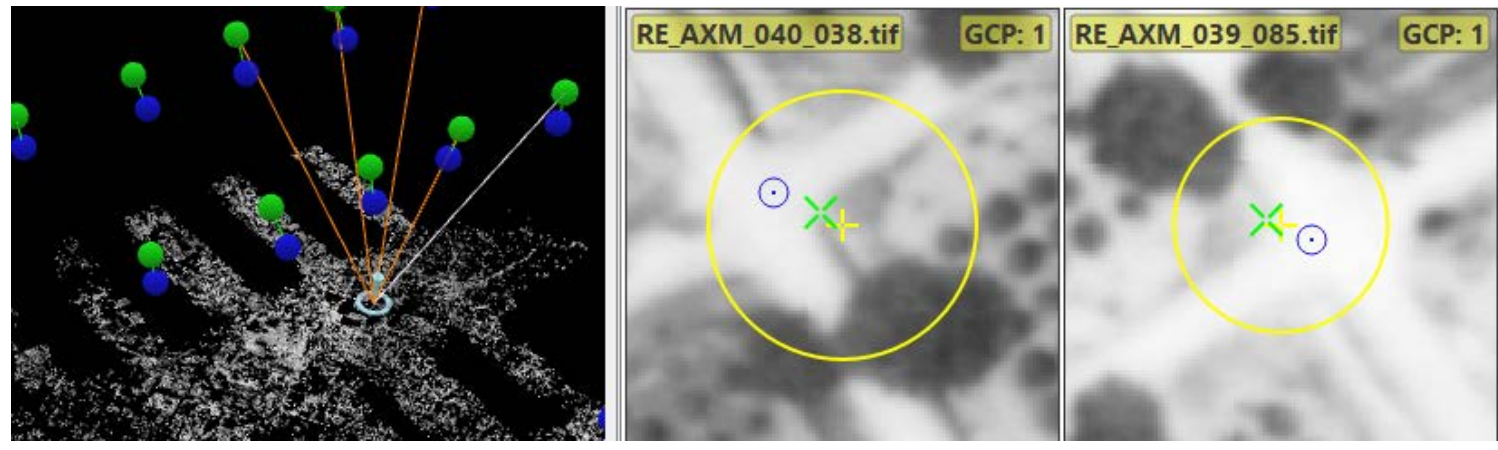

Figure 5-8: Tagging control points in imagery

The reference point is shown highlighted on the left. In each image on the right the estimation of the reference point's position is shown in blue, with the user's manual tag in yellow and the auto refined position in green. Once the reference points were tagged in the imagery the processing solution was revised using the Reoptimize tool and then a new processing report was created using the Regenerate Quality Report function.

With ground control added, the quality report provided valuable information on the quality of the processing solution. The RMSE error values were used to determine if there were inconsistencies in the reference points used. Any issues resulted in rechecking 
the points captured in Section 5.3 for errors and switching which ground control points were used in the final solution. The results of this analysis are discussed in detail in Section 6. Once the reference points were acceptable the solution was completely reprocessed to obtain a revised DSM and orthomosaic using the most accurate results.

\subsubsection{Phase 3 - Refining Processing Solution}

Once the first two phases of processing were completed the overall quality of the processing products were evaluated for elevation artifacts. By examining the generated DSM it was evident that there were significant elevation spikes caused by matching errors between overlapping images. These errors primarily occurred in farmland where orange groves presented as uniformly spaced dots as shown in Figure 5-9.

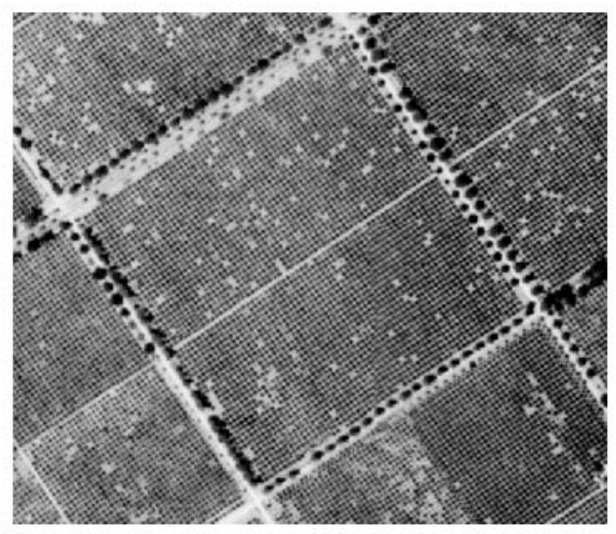

Figure 5-9: Image matching errors

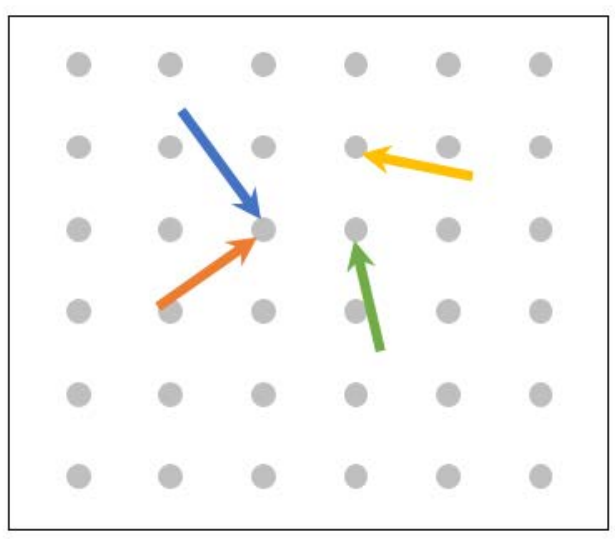

Misaligned Automatic Tie Point

The errors were caused by Pix4D Professional being unable to precisely determine the matched location from multiple overlapping images during processing. In Figure 5-9 each overlapping image is represented by a different color of arrow. The arrows should point to the same location, but instead are slightly misaligned. The inconsistent match for the automatic tie points created uncertainty which manifested as elevation artifacts in the DSM. These elevation artifacts could be as large as 30m high as shown in Figure 5-10.

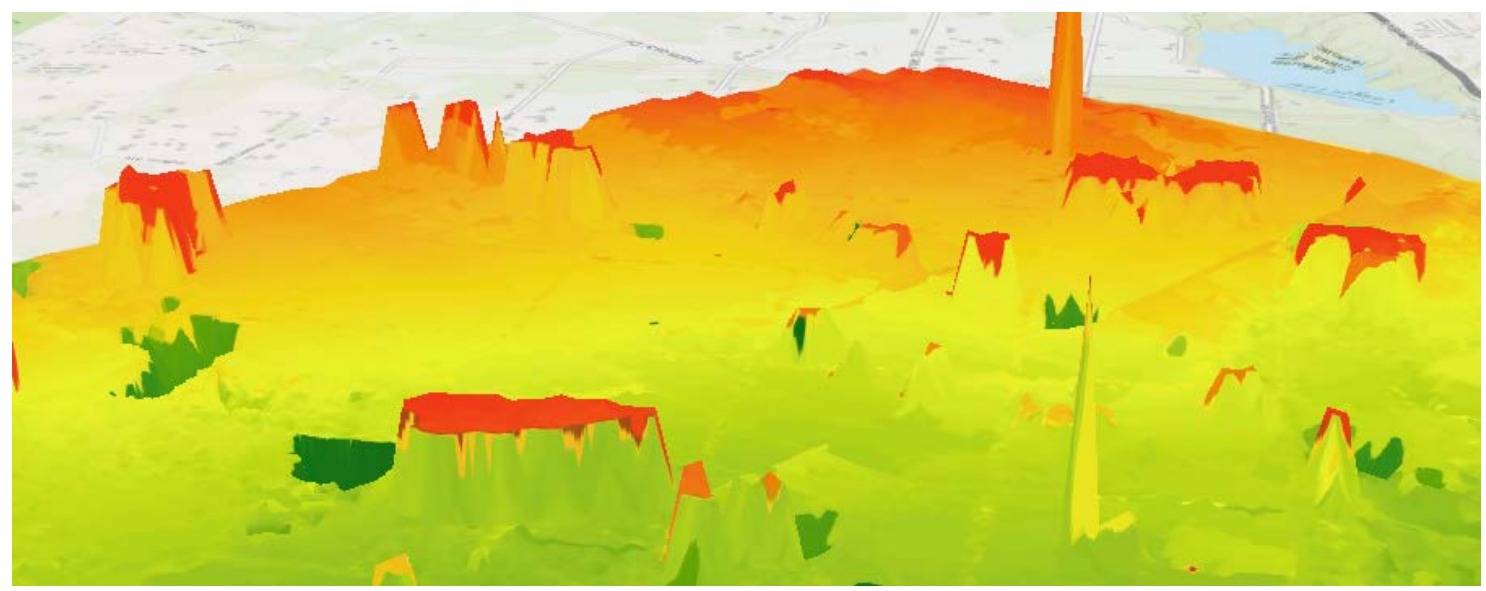

Figure 5-10: Elevation spikes caused by image mismatches 
The elevation spikes occurred over repetitive patterns in the terrain, these were primarily located over farm fields with symmetrical orange groves. They could be minimized in processing by adding manual tie points in each affected area. Manual tie points were user selected matched points between overlapping images that did not have a known coordinate and were not considered either check or control points. They forced the solution together at the affected areas. The process to add manual tie points was similar to tagging a reference point in Figure 5-8. Other raster processing strategies were employed to minimize these artifacts after processing but this method was the most effective within Pix4D Professional to improve the data quality.

Once some manual tie points were added the solution was reprocessed and a new coarse DSM and orthomosaic generated. The new product was then checked to evaluate whether more manual tie points were required to further improve the solution. With each processing iteration, the Quality Report was checked for improvements or degradations. Updates to the ground control or other settings were performed accordingly if the results changed. For small areas this method worked effectively. Once a satisfactory DSM was produced, one last processing session was run to create final data products at a cell size of $2 \mathrm{~m}$.

The products from Pix4D were further refined in ArcGIS Pro to improve the horizontal accuracy of the orthomosaic and remove elevation artifacts from the DSM across a larger area. The final orthomosaic was georeferenced using the Image Analysis tools in ArcGIS Pro to the target control points. The georeferencing process improved the horizontal positioning accuracy of the orthomosaic, so that it could meet National Map Accuracy standards.

Elevation artifacts were identified and reduced using the Raster Calculator. Areas that differed from the reference surface by more than $25 \mathrm{~m}$ vertically were identified and eliminated. The elevation from the reference surface was substituted in the affected areas using the Raster Calculator.

\subsection{Generate Analysis Products}

After processing in Pix 4D and ArcGIS Pro was completed the output products were prepared for final analysis in ArcGIS Pro. The computed DSM surface was first compared to the reference DEM from USGS using the Raster Calculator. The computed surface was subtracted by the reference surface to create an elevation surface of the difference from 1938 to 2016, as shown in Figure 5-11.

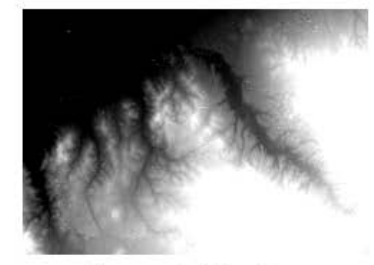

Computed Surface

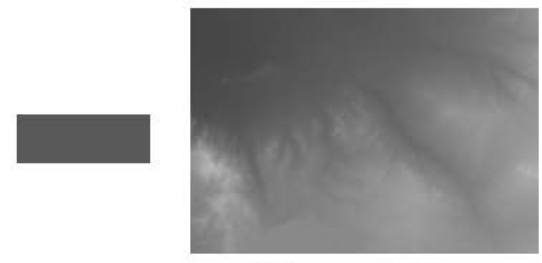

Reference Surface

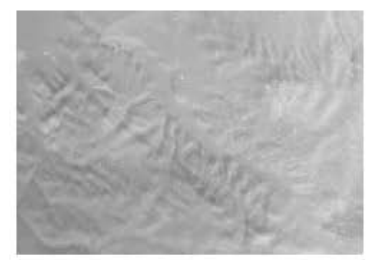

Difference Surface

Figure 5-11: Calculating the difference surface

The raster of the difference was then colorized to indicate areas of change. Areas of high vertical change were examined to determine potential causes and the computed 
orthomosaic was added to aid in this process and to visually verify image quality. The products were also incorporated into map scenes to create 3D visualizations of the terrain as it appeared in 1938. The elevation and imagery for the scenes were derived from the Pix4D processing session, and the colorization customized in ArcGIS Pro. Areas of interest from this analysis are shown in Section 6.

\subsection{Summary}

The implementation of this project the use of Python, ArcGIS Pro and Pix4D

Professional. The Python script standardized the input scanned imagery. ArcGIS Pro was used to create and manage GIS data and to create the final mapping and information products. Pix4D Professional performed the image processing and quality analysis. The methodology for using these technologies was reviewed in this chapter, and the results and analysis of that process are discussed in Chapter 6. 


\section{Chapter 6 - Results and Analysis}

The purpose of this project was to evaluate whether the computer vision photogrammetric method could expediently produce an orthomosaic and DSM from a collection of historical aerial images to the desired accuracy. This chapter describes the results and analysis of the workflow to standardize the imagery, process it in Pix4D Professional, and produce meaningful information products.

Section 6.1 provides an accuracy assessment of each stage of the project, from the standardization Python script, to the initial pre-processing for flight integrity, to the accuracy of the output orthomosaic and DSM. Section 6.2 discusses project error sources and their effects. Section 6.3 reviews features of interest in the project site that demonstrated the value of performing the work. Section 6.4 discusses the processing duration and whether this methodology effectively saved time over other manual forms of processing.

\subsection{Accuracy Assessments}

There were no formal requirements from the client to meet a specific horizontal or vertical accuracy, due to the uncertainty about whether this method would work effectively. However, as an informal target the accuracy standards defined in the original project completed by Buchwald were used "at a scale of 1:20,000, one fiftieth of an inch on the map would be 33.333 feet or 10.16 meters on the ground” (Buchwald, p. 37) per National Map Accuracy Standards for $90 \%$ of the points evaluated. This was a consideration at all stages of the project starting with the image standardization process.

\subsubsection{Standardization Python Script}

The goal of the Python script was to standardize the input imagery for size and rotation, and ensure that the image center was consistent across all images. Correcting these images was critical because Pix4D assumed that each image with a different size was generated by a different camera and would create a unique camera correction for each image otherwise. Ensuring that the image center was positioned in the same location guaranteed that the computed camera correction was uniformly applied across each image.

The average pixel offset of the original image center to its true image center (computed by the intersection of the fiducial marks) was 35.3 pixels. Each pixel represented a ground sampling distance (GSD) of approximately $45 \mathrm{~cm}$ as computed by Pix4D; this meant that on average the image center was $15.9 \mathrm{~m}$ from the true image center when related to the ground. If the original images were used in processing without standardization, any camera correction applied during processing would be applied disproportionately, causing data inaccuracy and processing failure. An attempt to process the original images was made without the corrections, and the result was a processing session that took twice as long and crashed the processing machine. The preliminary results using the original images indicated 25\% fewer match points, $10 \%$ fewer keypoints, and inconsistent image matching accuracy. After standardizing the images, the 
image centers were nearly the same across images differing on average by less than a pixel as shown in Table 4.

\section{Table 4. Calculated Pixel Offset of Image Center}

\begin{tabular}{|c|c|c|c|}
\hline & Min & Max & Average \\
\hline Original Images & 6.4 & 85.4 & 35.3 \\
\hline Standardized Images & 0.25 & 1.9 & 0.97 \\
\hline
\end{tabular}

The small uncertainty in the standardized images can be attributed to the difficulty in manually selecting the precise location of the fiducial marks in each image. Any image that had a calculated pixel offset of greater than approximately two pixels was reprocessed to reduce the offset. This pixel cutoff was determined heuristically after recalibrating a few images and reprocessing them. A difference of under two pixels resulted in less than $5 \mathrm{~cm}$ of change in the final product accuracy. The results for each image standardization are shown in Appendix A.

\subsubsection{Quality Report}

As part of processing, Pix4D Professional generates a quality report that can be used to validate the source data and its integrity. As discussed in Section 5.4.1, it was important to validate that the images had all been incorporated into the solution correctly, and that there were sufficient keypoints and matches between adjacent images generated. All 28 of the test images were used and an average of 79,645 keypoints and 18,915 matches per calibrated image were found. This exceeded the minimum thresholds of 10,000 keypoints and 1,000 matches, indicating the source data were viable for use in Pix4D.

The report also provides information on the image overlap that was used to identify areas of the project that would suffer accuracy issues in processing. The image overlap for the project is shown in Figure 6-1. 


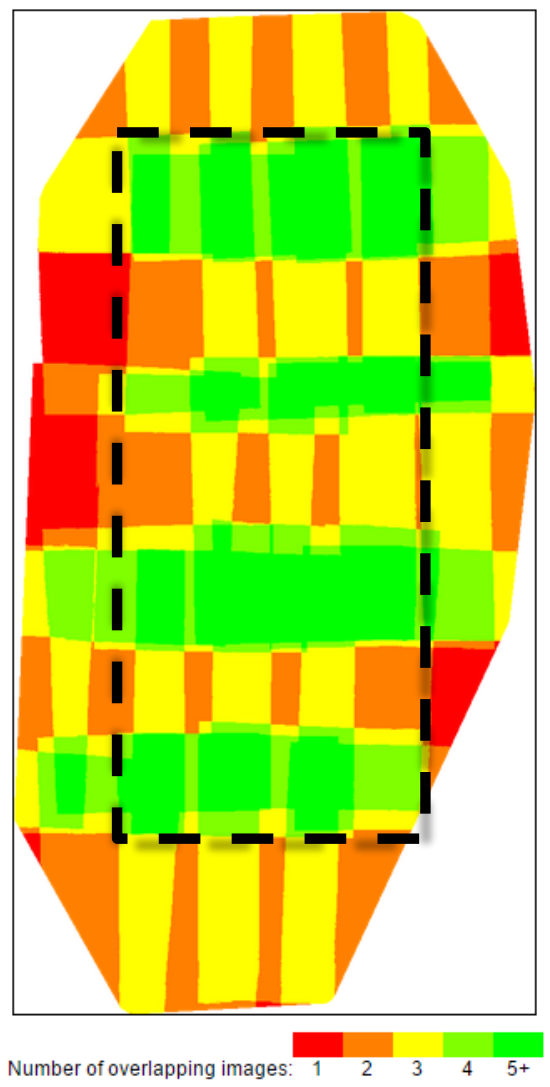

Figure 6-1: Image overlap of the project area

A minimum of two image overlap is required to process a result in Pix4D, but three overlapping images are the preferred minimum and the default setting in the software. Analyzing Figure 6-1 reveals that much of the project area is covered in only two or three image overlap, an early indicator of the challenges of processing a project area such as this. The only five or more image overlap occurs at the intersection of the image ends between flight lines. While this is a positive, to have some overlap with more images, these locations are often worse quality due to being blurry or distorted in the film image. Many of the overlap and image issues occurred at the edges of the project area, because of this the effective processing area for analysis was constrained to the black rectangle shown in Figure 6-1. The impact of the image overlap on the project outcome will be discussed in more detail in Section 6.2.1.

The quality report contains additional information on computed uncertainties in the camera position, orientation, focal length and principle point, but because these values are based on estimates by Pix4D and further optimized using only the input images through self-calibration, these statistics are not useful in analysis as the results vary with each processing session. For example, the optimized focal length after processing is $28.529 \mathrm{~mm}$ but if a smaller selection of only 15 images is used, the focal length is computed as $25.89 \mathrm{~mm}$. For the project, the focal length is listed as $20.96 \mathrm{~cm}$ (University of California, 2012), indicating the initial default value in Pix4D of 20cm was closer than the computed value after processing. When analyzing these reports under that context the 
optimized details of the camera should not be considered valid or used in analysis outside of the product that produced the results.

\subsubsection{Orthorectified Image Mosaic}

Despite the uncertainty of the source images, the lack of camera calibration information and the minimal image overlap Pix4D Professional produced a complete, seamless, color balanced image mosaic using the 28 input images. A small sample of the mosaic at the intersection point of over 10 images is shown in Figure 6-2 at a 1:50,000 scale.

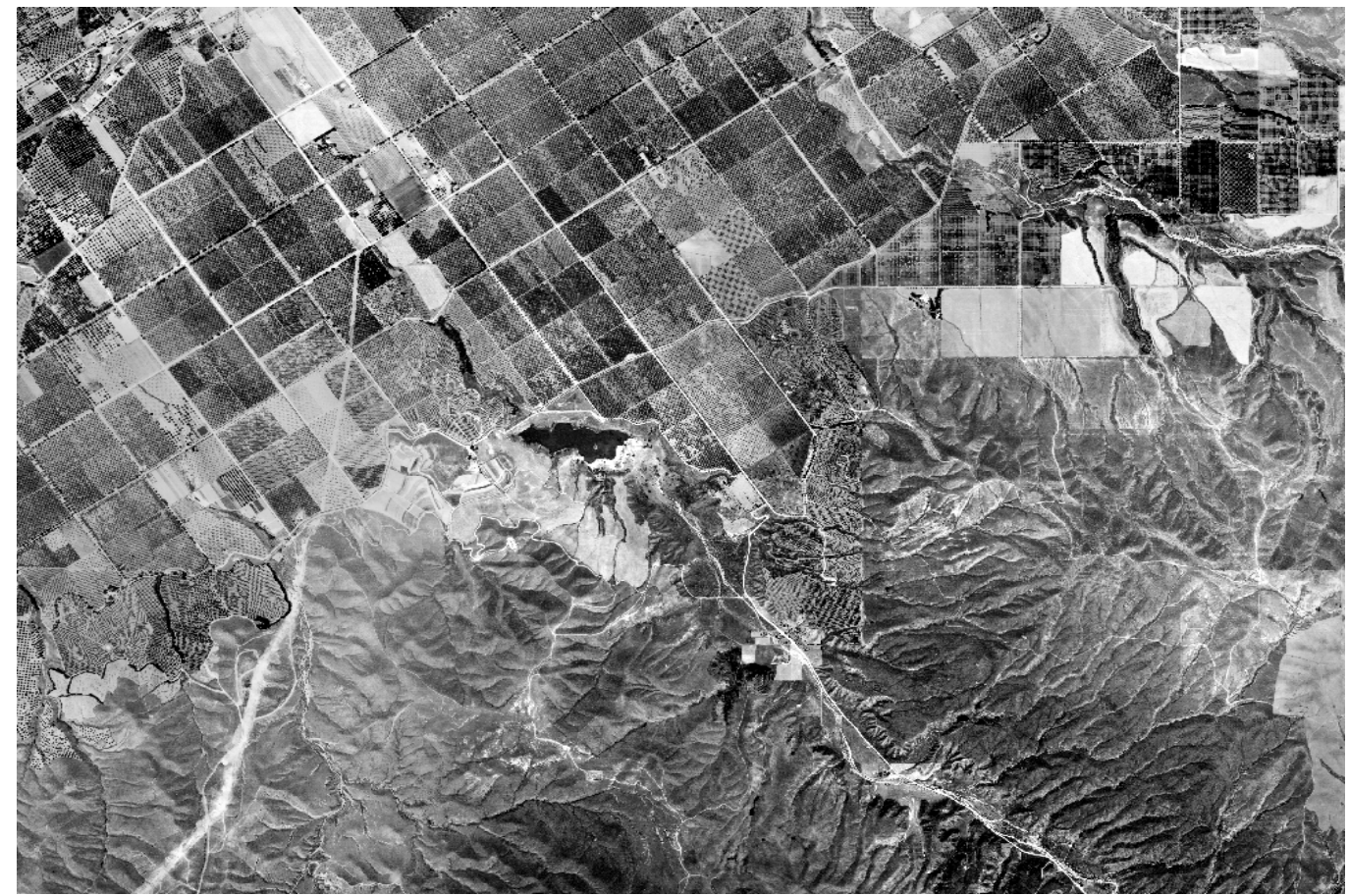

Figure 6-2: Orthorectified image mosaic 1:50,000

The mosaic shows no edges of the individual source images, it shows excellent color balancing and any image artifacts (primarily in farmland) are unnoticed by a viewer. The result is striking particularly when considering the number of overlapping images used to create this area as shown in Figure 6-3. 


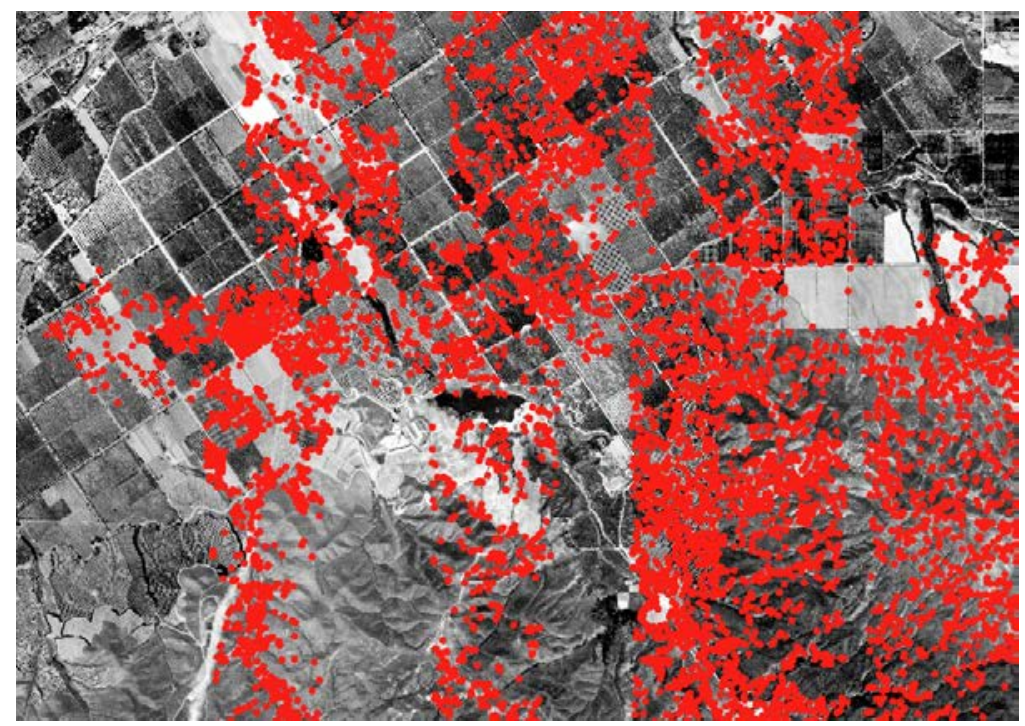

Figure 6-3: Matched keypoints from three or more images

Each red dot represents a matched keypoint between three or more images. There are over 10 images that compromise Figures 6-2 and 6-3 but to the naked eye at this, and even lower scales, such as 1:5000, it is very difficult to distinguish them. The extent of the project area of 28 images was mosaiced together with a similar level of visual continuity through the entire project area as shown in Figure 6-4.
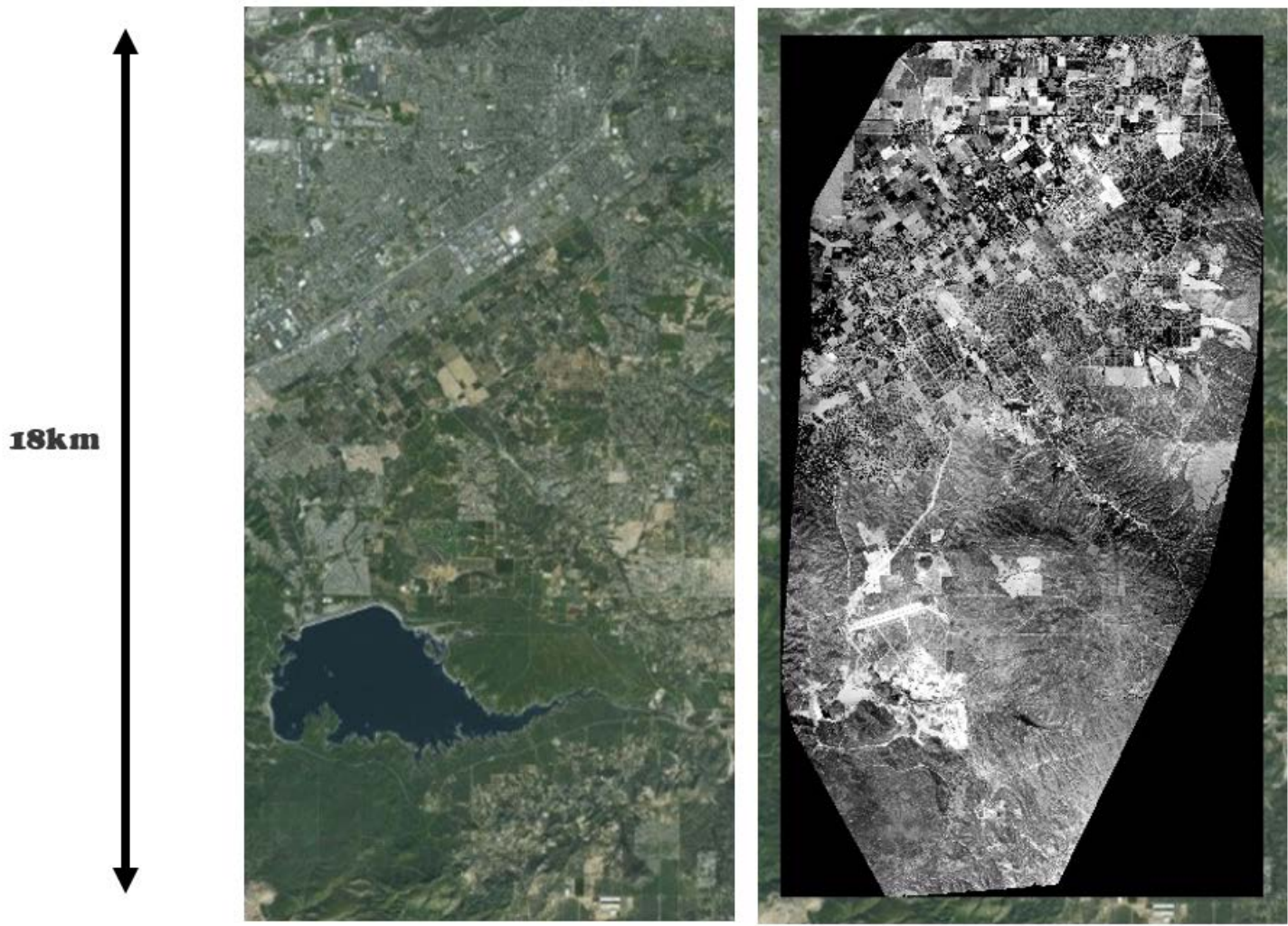

Figure 6-4: USGS basemap (left) and processed orthomosaic (right) 
The output orthomosaic was approximately $18 \mathrm{~km}$ long and $8.5 \mathrm{~km}$ wide. The extent of the final mosaic did not cover the full extent of all 28 images because the output was based on the extent of the computed DSM. Only areas with two image overlap or more were used to create the DSM. Because of this there was a loss of approximately half an image of coverage on either side of the project area. A feature of note in the project is Lake Mathews, which is a manmade lake shown in the lower left of Figure 6-4. In 1938 the dam to create the lake was still under construction as seen in the processed orthomosaic. This lake represented a significant area of change in the project and played an important role in the resulting accuracy of the final data products discussed later in this chapter. Other features such as mountains and hills were mosaiced seamlessly as shown below in Figure 6-5.

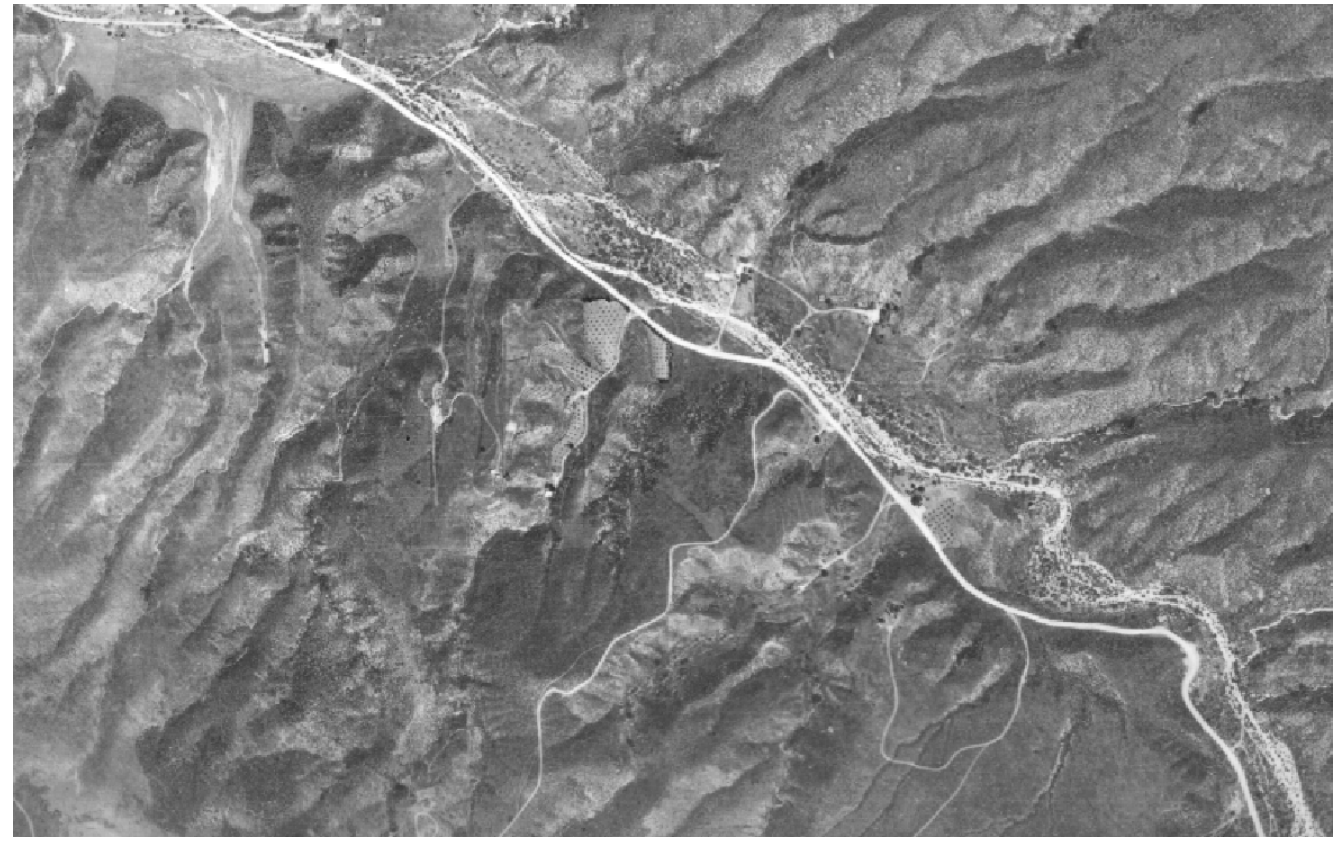

Figure 6-5: Continuous hilly range

The hills show minimal image distortions due to elevation artifacts in the DSM. This can be a problem with some methods of georeferencing that use a network of ground control points instead of a complete DSM. The DSM generated by Pix 4D was sufficiently continuous to avoid many of these issues over areas of rapid elevation change. This was not true over many farm fields in the project site, as shown in Figure 64 which shows an image section of a citrus grove. 


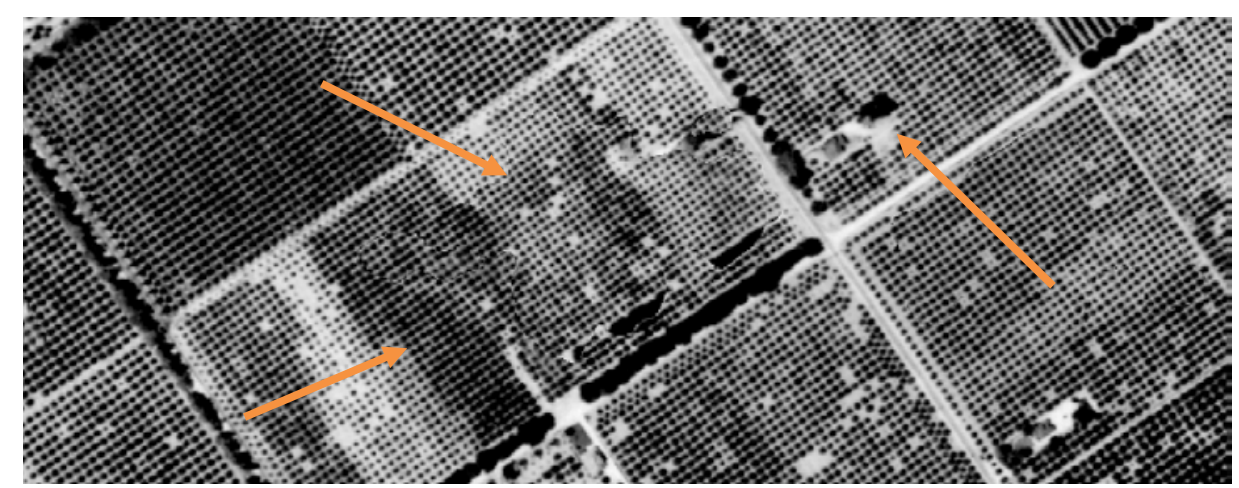

Figure 6-6: Elevation artifacts over farmland

The uniform patterns of orange groves caused issues in the automatic matching of overlapping image points which resulted in large elevation artifacts (over 30m vertically) in the DSM. When this DSM was applied in the image orthorectification process the affected areas in the mosaic were shown to have distortions as indicated in Figure 6-4. The terrain looks warped and the tree line near the road is partially duplicated in the farm field. These distortions are often difficult to see, and depending on the view scale of the imagery are undetectable. At a 1:10,000 scale they are invisible, but at 1:5000 or closer they are noticeable if left uncorrected.

The horizontal accuracy of the output orthorectified image mosaic was established using reference points and assessed using checkpoints that were established in the project area. The optimal location for the reference points was found to be in areas of high image overlap (four or more images) and where the check points were interspersed between control points. The graphical results displayed in the following sections show a selection of the check points defined for the project. The complete quality reports with supporting residuals for each processing session are shown in Appendix B.

Initial processing attempts focused on evaluating reference points across the project area. Due to the uncertainty associated with both the horizontal and vertical positions of each reference point it was important to try to identify any outliers. After a processing session was completed the horizontal offsets between the computed coordinate and the source coordinate were calculated as residuals. The project area was processed first by holding a selection of 3D control over the site and comparing the residuals of all the check points. The horizontal residuals for each check and control point are shown in Figure 6-7 on the left, and the elevation difference between the computed and reference surface on the right. 

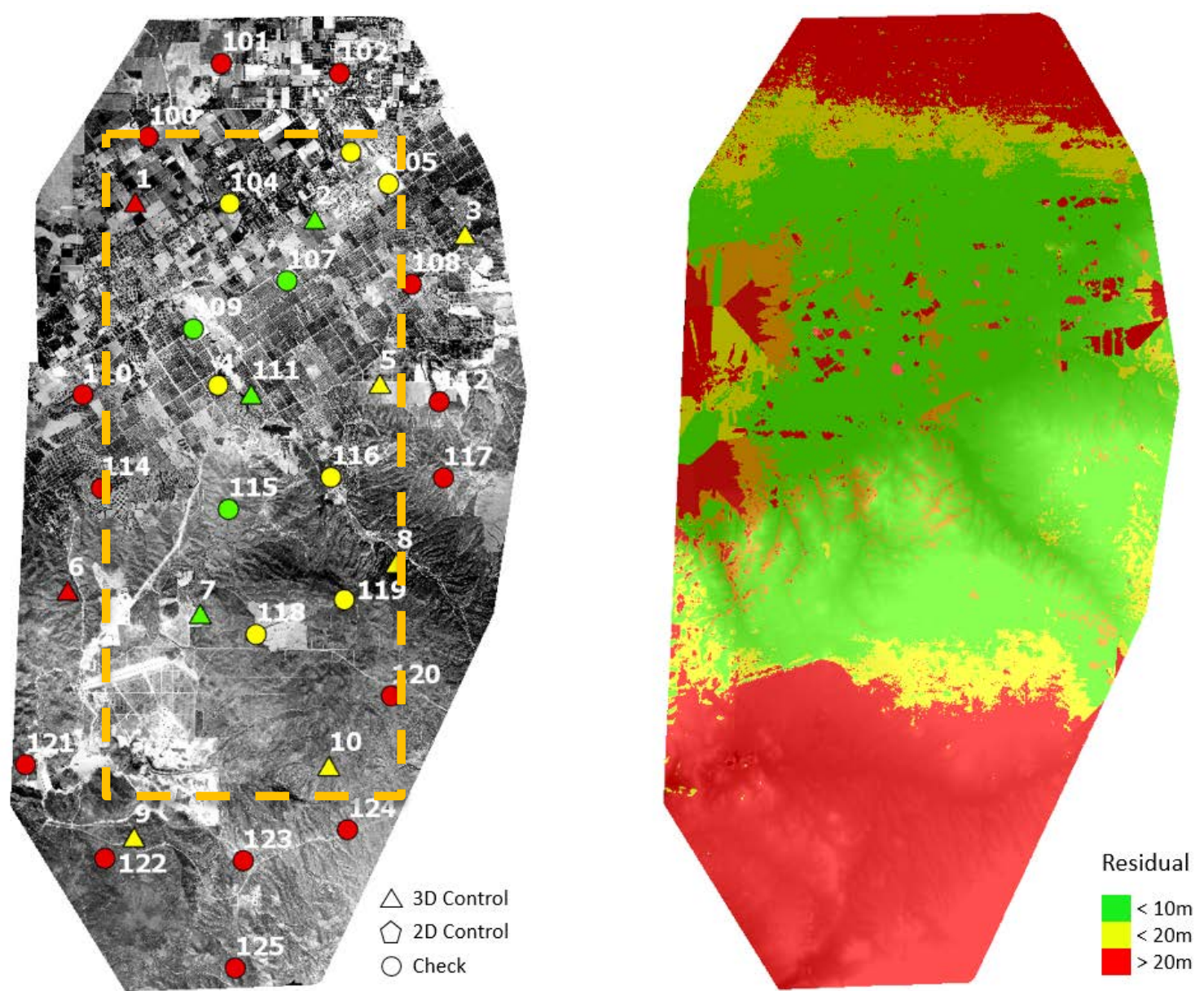

Figure 6-7: Horizontal residuals (left) and elevation difference (right)

The results indicate that there is a significant drop in horizontal and vertical accuracy outside the area enclosed by the orange rectangle representing the effective processing area. The solution quality degrades the farther the check point is from a control point on the edges of the project area due to extrapolation of the solution, poor image overlap or insufficient control point coverage. The horizontal accuracy within the effective boundary of the control points also does not consistently meet the desired $10.16 \mathrm{~m}$ accuracy but is generally under $20 \mathrm{~m}$.

The elevation differences increase dramatically to the south where the effect that Lake Mathews has on the solution is evident. No reference points could be established over the Lake Mathews area, and the surrounding areas had few recognizable features in areas of high overlap. This resulted in few points being available and only control point 9 could be established as a consistent point of reference. Thus, the south end had a weak solution and consistently higher than $20 \mathrm{~m}$ horizontal residuals.

The area around the lake also showed high elevation change, which is not an error but an actual product of the terrain being substantially different between 1938 and the present day. The USGS DEM used as reference had an elevation over the lake set at the water surface, while the generated DSM from Pix4D approximated ground in 1938. The vertical residuals in that area (despite being significant) may be accurate and 
representative of true differences between now and then. Lake Mathews has an average depth of $45 \mathrm{~m}$ and the difference in that area is often over $40 \mathrm{~m}$.

The erratic elevation offsets at the edges to the east are due to interpolation of the DSM between the areas of low image overlap. The lowered accuracy to the north and south is due to extrapolation of the processing solution past the control points. These poor elevation results would affect the quality and horizontal accuracy of the orthomosaic which is seen in the check points for these areas. Since the results of this session did not meet the preferred horizontal accuracy standards, the integrity of the control points was verified independently by georeferencing the generated orthoimage in Arcmap. The results are shown in Figure 6-8.

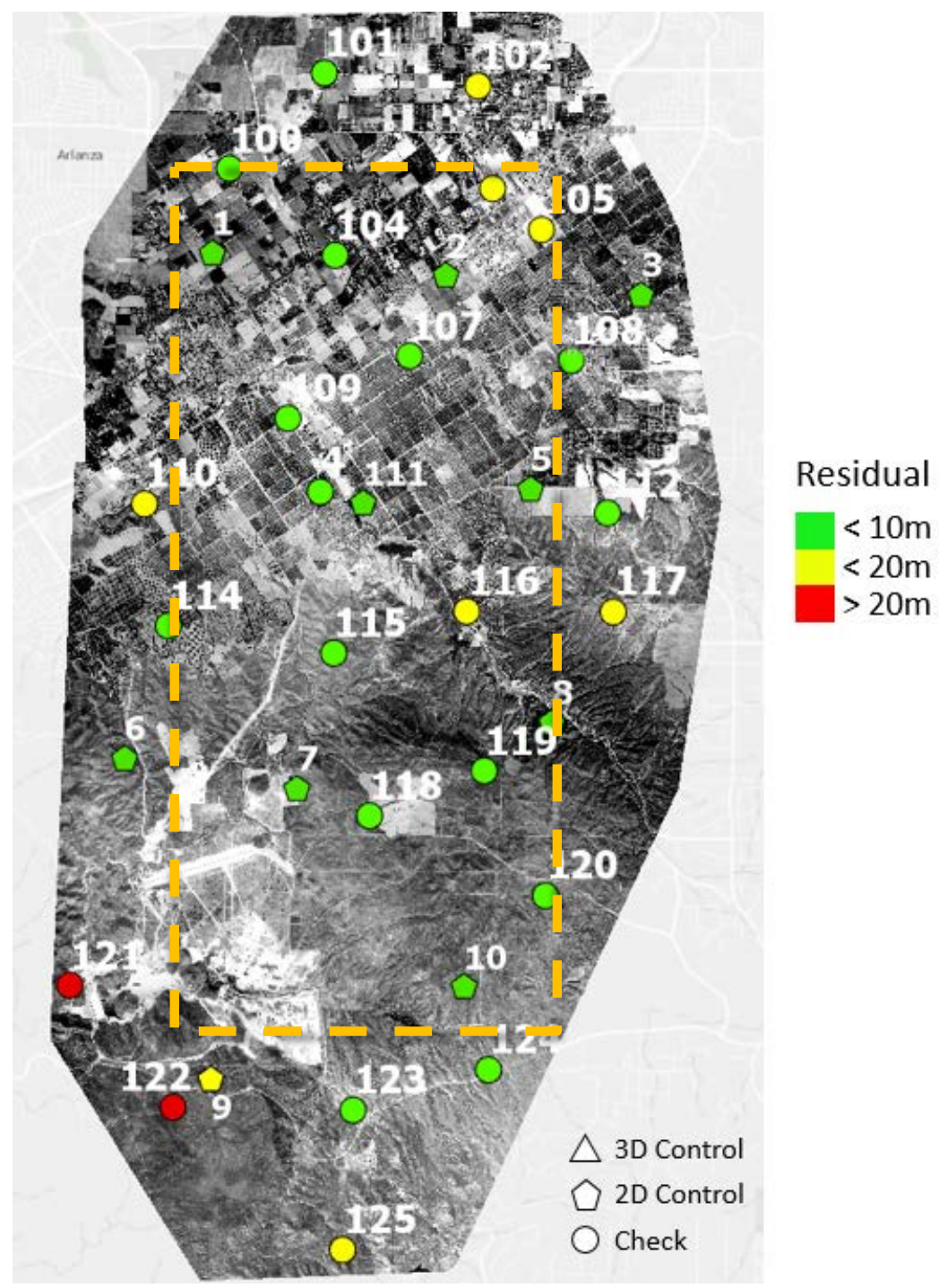

Figure 6-8: Georeferenced orthomosaic

Georeferencing the mosaic using the same control points produced a highly accurate result. The majority of the project area met the horizontal accuracy standard of $10.16 \mathrm{~m}$ and verified the horizontal integrity of the gathered control points in the north. Some issues are still present outside of the effective processing area (orange rectangle) covered 
by the control points and around Lake Mathews with points 121, 122, and the control point 9. This indicates that additional control was required to refine the solution, however, this was difficult to find due to the uniformity of the terrain. If control was found then the whole project area would likely meet the horizontal accuracy target set out in the National Map Accuracy Standards.

This method of further georeferencing the Pix4D orthomosaic provides an alternate way to quickly produce an accurate final orthomosaic as opposed to continuing to iteratively process using only Pix4D. It is a valuable method in situations like this where the project terrain is challenging. It is faster and more accurate to produce the mosaic using this method, than to adjust different combinations of control points in Pix4D. The georeferencing transformation could be applied to the DSM to refine its horizontal position.

The result in Figure 6-8 verifies the overall integrity of the control points horizontally to the north and indicates that many of the issues in horizontal accuracy observed in the original 3D processing session were due to uncertainties in the vertical component of the ground control's position and its effect on both the horizontal and vertical solution in processing.

More 3D control points were added to the solution to evaluate the effects on overall horizontal and vertical accuracy and improve the solution quality within Pix4D. The added 3D control points did refine the vertical solution but contributed uncertainties to the computed horizontal positions as shown in a selection of the check and control points displayed in Figure 6-9.
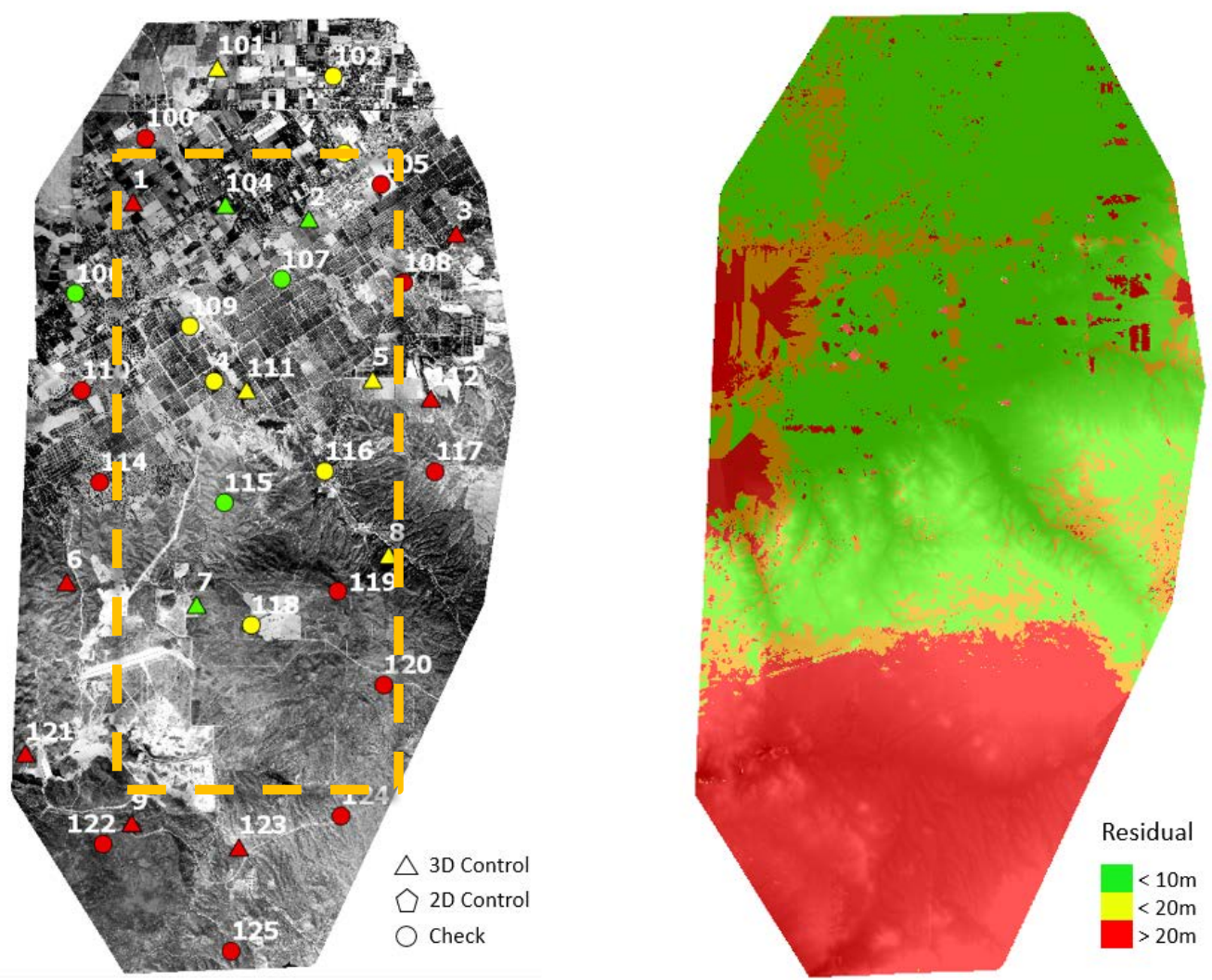

Figure 6-9: Horizontal residuals (left) and elevation difference using more control points (right) 
To the north the difference in elevation between the computed and reference surface improves dramatically while the difference to the south remains high even with additional control points. The isolated areas of change to the west are areas created by Pix4D interpolation and would be resolved by adding more images to the solution in those areas. As discussed previously, a large portion of that difference is legitimately caused by Lake Mathews but there is no subsequent improvement to the south of the lake. The horizontal residuals for the project become more evenly spread, but in the effective project area (orange rectangle) there are fewer points that meet the $10.16 \mathrm{~m}$ accuracy target. This indicates that adding more 3D control does improve the alignment of the vertical solution, but the added uncertainty has a negative effect on the horizontal alignment. This affects the accuracy of the orthomosaic, but also the horizontal accuracy of the DSM and ability to use it effectively in analysis. Normally the vertical value for a control point could be verified but because of the uncertainty associated with using historical images that is not possible as there is no elevation control from that time-period.

If there were additional higher accurate 3D control points dispersed through the project area it would be preferred to use them in the solution as they would be able to control the area like in the center of the project area in Figure 6-7. But in this situation where control in the overlap was difficult to obtain, and had high uncertainty, it was a challenge to find an optimal processing path. Reprocessing with different combinations of control was also difficult to manage from a productivity perspective as each control point took time to find, tag appropriately in Pix4D and evaluate properly in processing.

An alternate method to obtain only the highly accurate orthomosaic would be to perform a rough processing session with some 3D control and then georeference that product outside Pix4D. This alternate strategy met the desired horizontal accuracy target of $10.16 \mathrm{~m}$ for the project scale. Details on the potential errors that contributed to the uncertainty in the processing solutions reviewed in this section are discussed in more detail in Section 6.2.

\subsubsection{Digital Surface Model}

The digital surface model created by triangulating the aerial imagery, contained an impressive amount of terrain detail, considering the constraints of the source data provided. Visual inspection quickly identifies key features such as the hills to the south as shown in Figure 6-10. 


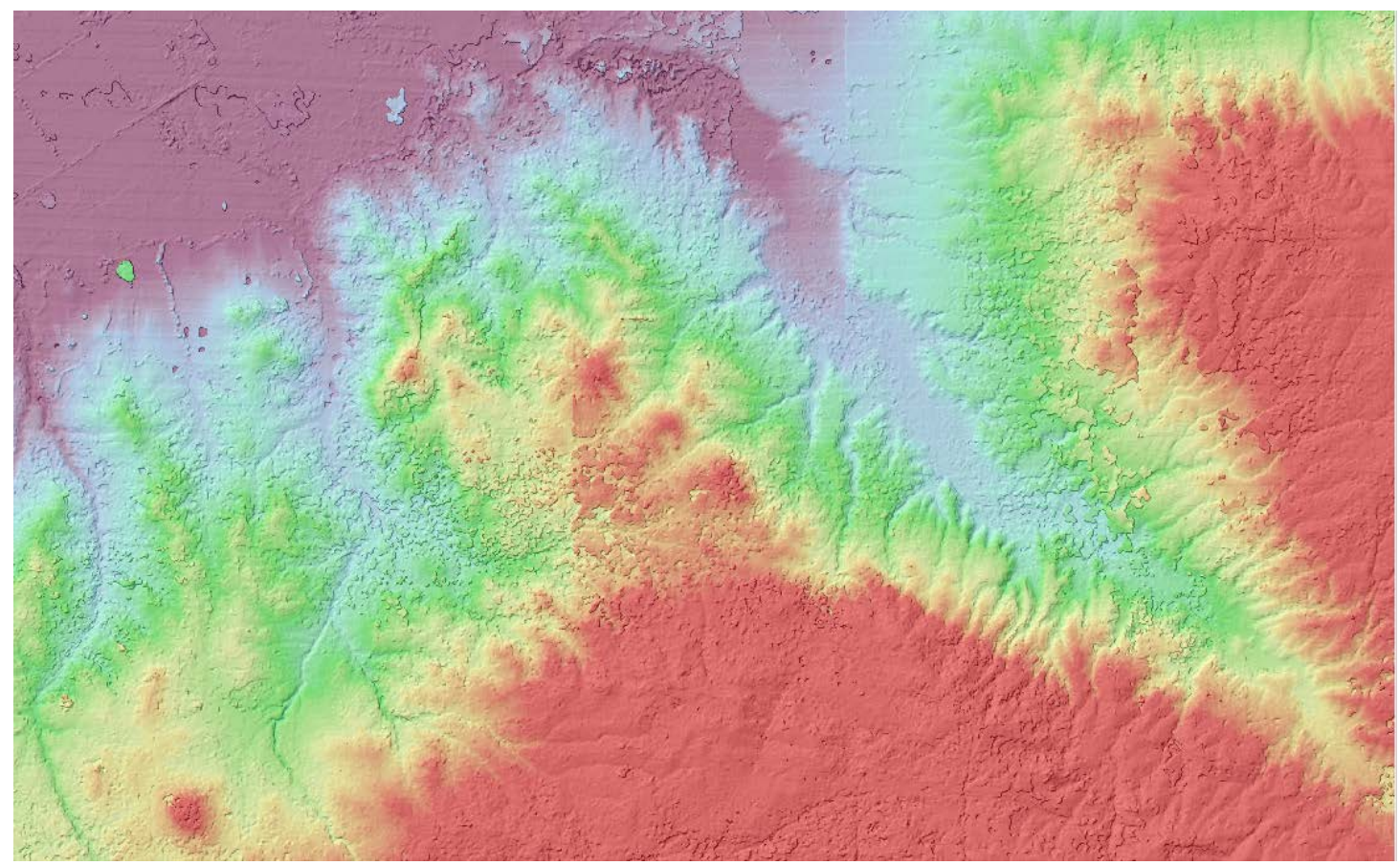

Figure 6-10: Digital surface model with hillshade overlay

The steepness of the cliff face is apparent and the detailed grooves of the hillside stand out immediately in the DSM. While the hillshade for the area creates some noise in the image the trend of the countryside is clear. This product could be used in baseline or trend analysis depending on the scale. When the whole project area is compared to a current reference surface some key differences between the past and the present are observed as shown in Figure 6-11. 

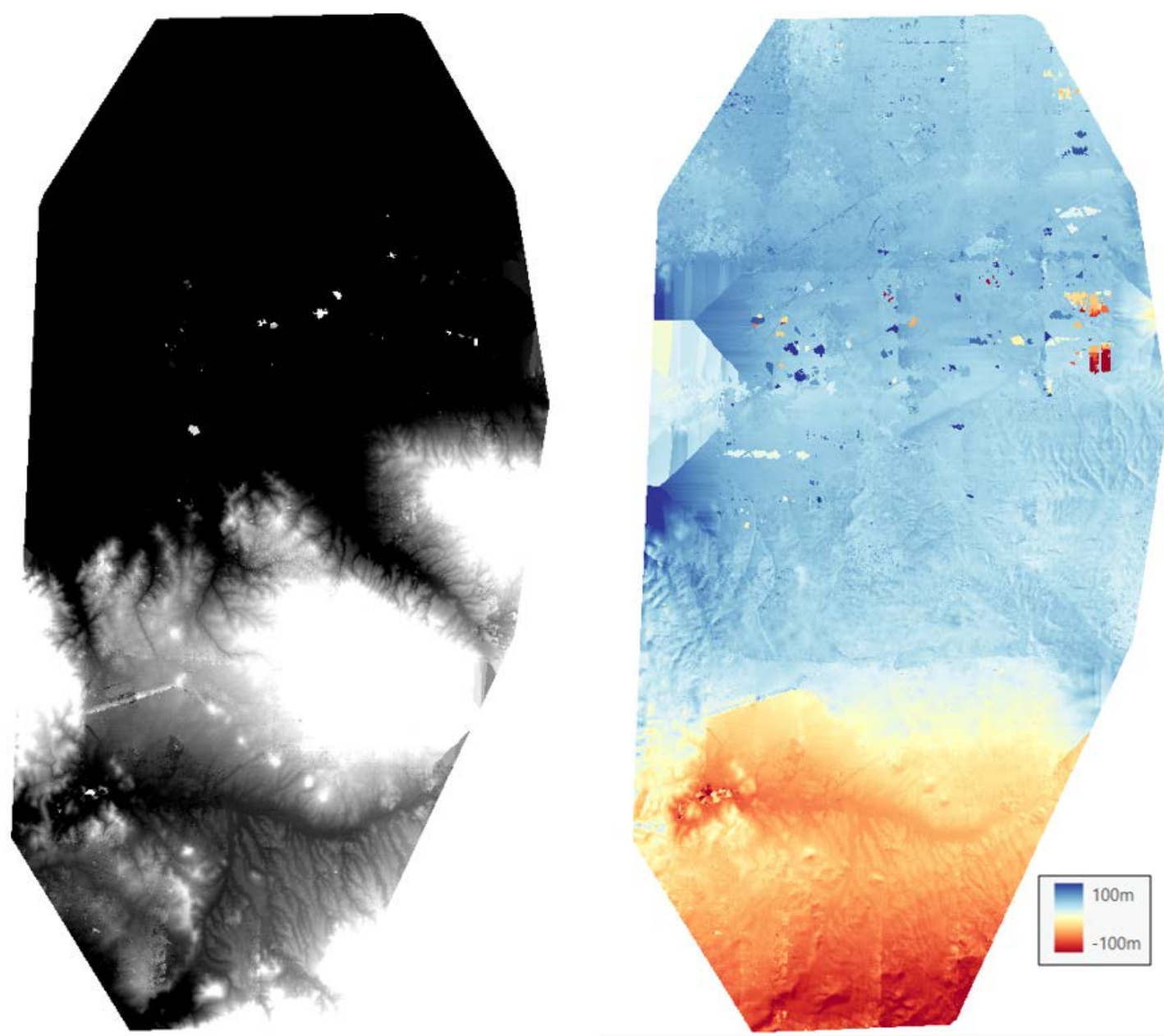

Figure 6-11: Computed DSM (left) and difference between surface between DSM and reference surface (right)

The crests of the hills are still easily discernable, as well as the drainage channels around the area that will become Lake Mathews. As discussed in the last section there are sharp drop-offs in the accuracy of the elevation comparison to the west and south edges of the project due to the surface being extrapolated or interpolated. The difference between the past and the present is under $10 \mathrm{~m}$ over stable terrain when additional control points were used. The computed surface does contain many artifacts in the farmland area illustrated in the north as the small areas of sharp elevation change. These were created by the keypoint mismatches in processing. To correct them, manual tie points were inserted into the processing solution, but the results were often mixed as shown in Figure 6-12 below. 


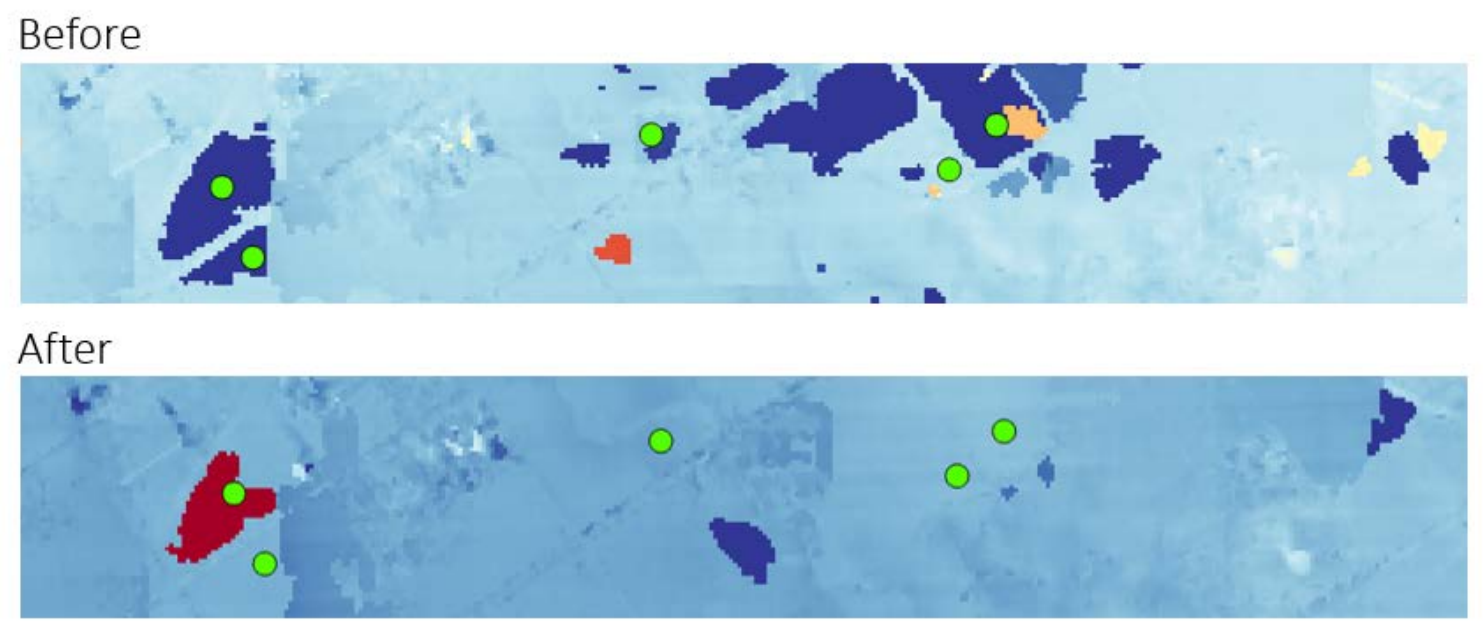

Figure 6-12: Effects of adding manual tie points on elevation artifacts

The manual tie points were inserted into areas that experienced elevation artifacts to assist the matching process and correct artifacts in farmland areas. Often, they improved the solution as shown in the Figure 6-12, but the results were not perfect and sometimes the manual tie points would have no effect or a new elevation artifact would be created in the same area after a processing session. Adding manual tie points was also a very timeconsuming process as the areas had to be identified, and then a point found in Pix4D that matched across multiple images. What was difficult for the computer algorithms to do (find a common point in a field of similar features) was manually challenging for a human as well. This solution while effective in improving data quality required many iterations of effort to correct areas, and proved to not be a practical method of resolving the artifacts. Different processing methods were selected in Pix4D but none proved effective in resolving these issues, as the better methods required more precisely positioned image coordinates. Using the Raster Calculator in ArcGIS Pro it was possible to find these elevation artifacts by isolating specific offset ranges from the difference surface. Eg. Where the elevation surface differs by more than $25 \mathrm{~m}$. This is shown in Figure 6-13.

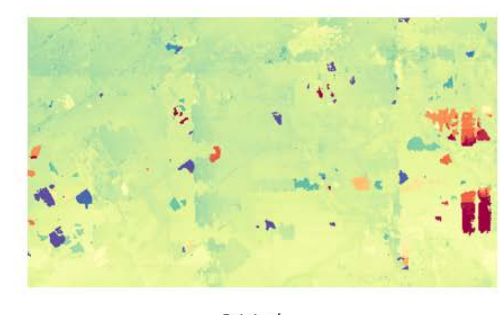

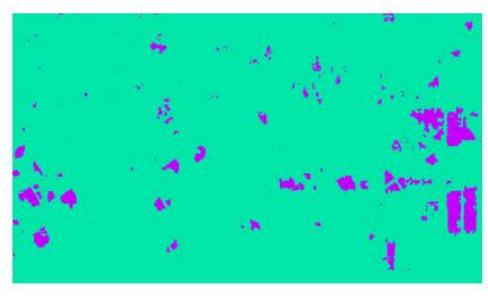

Identify Artifacts

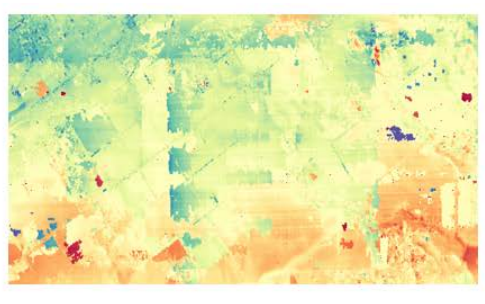

Corrected

Figure 6-13: Elevation artifacts isolated and corrected using the Raster Calculator in ArcGIS Pro

The Raster Calculator was used to swap out the elevations determined to be artifacts with either a null elevation or by substituting the elevation from the reference surface as seen in Figure 6-13. This worked well in small areas but could not easily be applied 
across the entire project area due to some of the elevation differences around Lake Mathews. Creating an automatic method to resolve these issues and incorporating the corrected DSM back into the orthoimage generation process was not in the scope of this project, but is discussed as one opportunity for additional study in Chapter 7.

\subsection{Error Sources and Effects}

There are many sources of error and uncertainty that contributed to the final solution. Some of these could be resolved in processing while others could not and were either caused by factors of the acquisition, source data, or processing method. This section reviews the major sources of error and uncertainty found over the course of the project with a brief analysis on the effects each had on the result.

\subsubsection{Image Quality and Matching}

In Section 6.1.2 the Quality Report was reviewed and the overlap was discussed. It indicated that the project area had enough overlap to be considered viable, but that the majority of the project area residing in only two or three image overlap. The lack of image overlap was partly caused by how the original survey was planned and executed as shown in Figure 6-14 below.

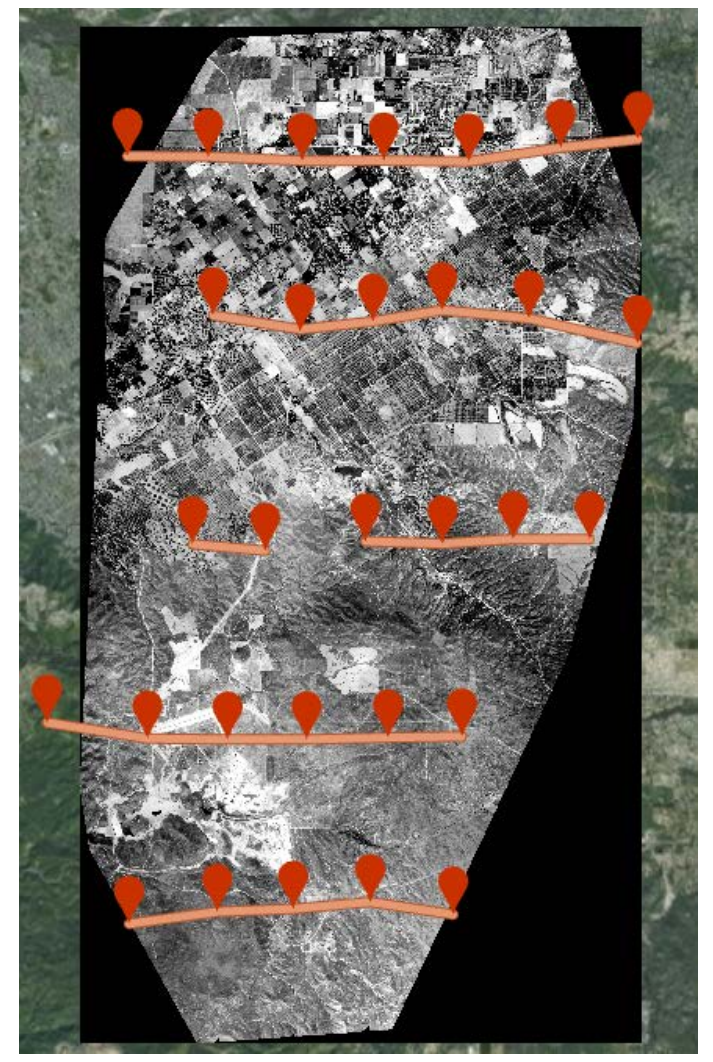

Figure 6-14: Project flight lines and image centers

The flight lines were often not flown straight and the spacing between lines is inconsistent. For example, the second flight line down is substantially closer to the top- 
flight line. These flight conditions contributed to the strained overlap shown in Figure 61 , where there was only very thin three or more image overlap in the center of the project.

The image quality was also not uniform across the scanned photograph. The upper and lower edges of the image could experience degradations in image quality. At the very edges features could be unintelligible as shown in Figure 6-15 below of two photos over the same location.

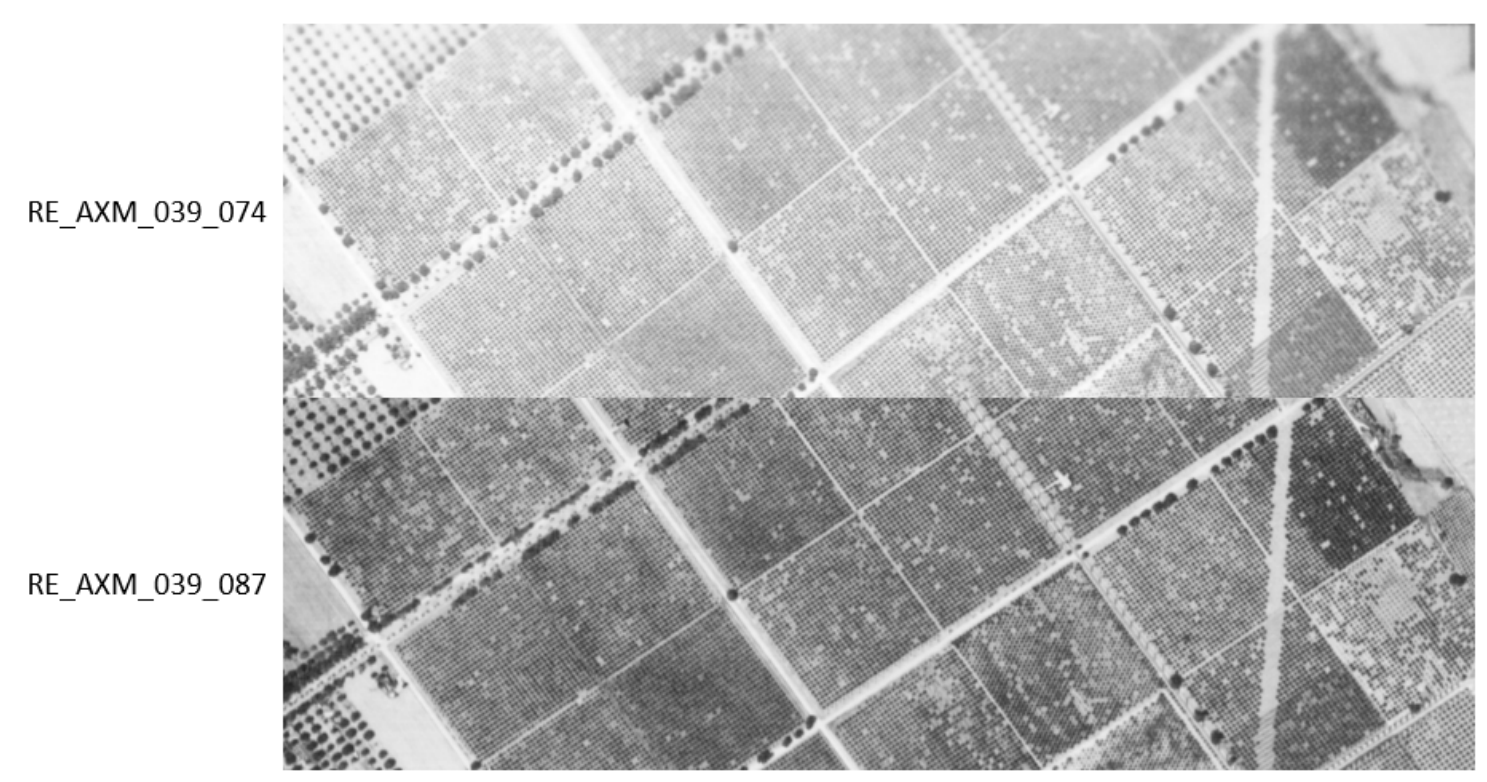

Figure 6-15: Differences in overlapping image quality

The image quality issues affected the ability of Pix4D to generate keypoints and to match those keypoints in some areas of the project using the default settings. These areas coincided with sections of the project area that experienced higher ground control differences such as point 106 in the initial processing session. They also appeared to contribute to the elevation artifacts over farm fields. The number of keypoints found could be increased in the Pix4D processing settings, but the processing duration increased dramatically (+20\%) and there was no substantial improvement to the solution's accuracy or the elevation artifacts. The additional keypoints and matches were created primarily in areas where there already were good image and keypoint definition. It is difficult to measure the impact of the flight overlap uncertainty but it did contribute to the overall uncertainty budget of the project.

\subsubsection{Image Centers}

Finding the image center in the source image was performed by the Python script, but correlating that image to the ground was a manual process. In some geographical areas it was challenging and the result was not highly accurate. The effect on the resulting processing solution was assumed to be minimal, but after adjustment of one image center by approximately $35 \mathrm{~m}$, the ground control and check points in the area experienced a positive correction of just under a meter. Corrections for the image centers were 
performed but even after a concerted effort to improve them some were too difficult to place with precision.

\subsubsection{Reference Points}

The collection and incorporation of ground control points and check points into the solution was one of the main sources of error and uncertainty in the project. Finding common points on the ground between 1938 and the present day was challenging, as they had to be found in areas of high image overlap. In some areas, points had to be selected in locations that were unideal due to the conditions on the ground as shown in Figure 6-16.

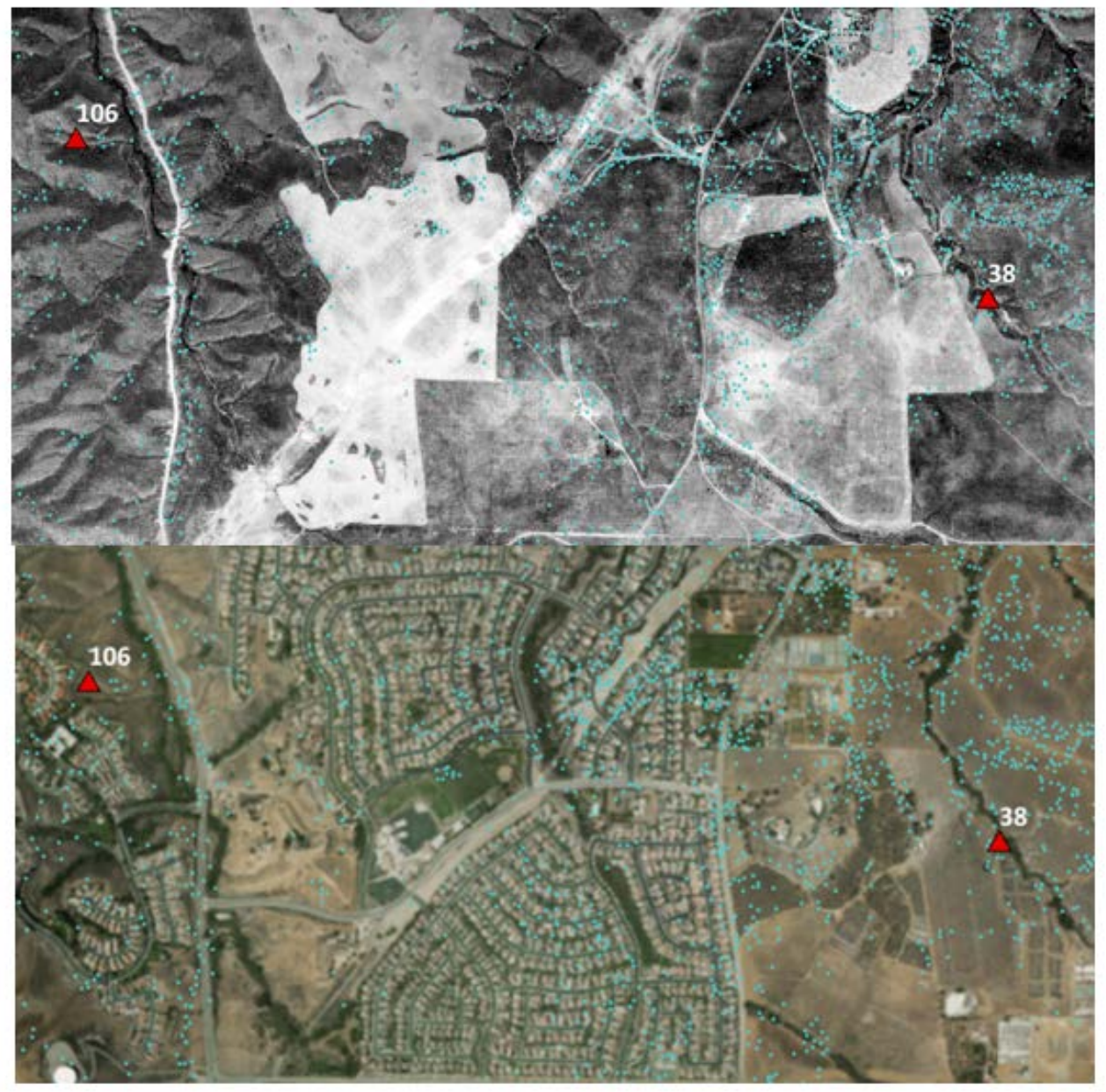

Figure 6-16: Determining reference points

In Figure 6-16 the preferred location for a control point would have been in the center of the image where the cluster of match points (blue) is shown, but because of the neighborhood that has been created there it was impossible to find a common point due to the landscaping of the area. Instead, fewer certain natural features were used at point 106 in the hills, and at point 38 around a patch of vegetation. While these features were identifiable, natural features were often difficult to pinpoint across multiple image sources due to shadowing, image resolution and camera positioning resulting in 
additional uncertainty. While more and better distributed ground control helped stabilize the overall solution as shown in section 6.1.3 it did not drive the overall accuracy of the solution into the target range. This was likely because while more ground control helped define each geographical region better, each point introduced new and conflicting levels of uncertainty in the processing solution. Fewer evenly spaced, higher quality ground were preferred, but even those were difficult to determine particularly in the far south and in the heavily urbanized area to the north.

One of the other challenges of trying to use reference control was accurately setting the horizontal and vertical accuracy of the reference points in Pix4D prior to processing. These settings had a pronounced effect on the solution from 10-30m horizontally and vertically depending on the location of the point in the project area. The initial accuracy was set to $2 \mathrm{~cm}$ by default in Pix4D, but for historical reference points this was an unreasonable accuracy. However, since there was no way to verify the measured accuracy of any point, determining a set of values had to be performed heuristically and values assumed for all of the control points. After numerous iterations of testing it was determined that $5 \mathrm{~m}$ horizontally and vertically was an effective value to use in processing. If fewer control points were used in the solution then some attempts of $2 \mathrm{~m}$ horizontally and $4 \mathrm{~m}$ vertically worked as well, but setting the values too tight with many control points in the solution resulted in significant busts in the project areas. It was another source of uncertainty in processing.

\subsection{Features of Interest}

Once processing was completed and the project area explored in more detail some features of interest were identified for further investigation. This section reviews a few of the more significant ones, while recommendations are made in Chapter 7 on items that could be explored in more detail.

\subsubsection{Manmade features}

Despite being 80 years old, some manmade features in Riverside still exist today and could be identified in the imagery and the DSM. In Figure 6-17 buildings from what is now the California Baptist University appear. They were constructed in the mid-1920s and 30s and taken over by the University in 1955. 

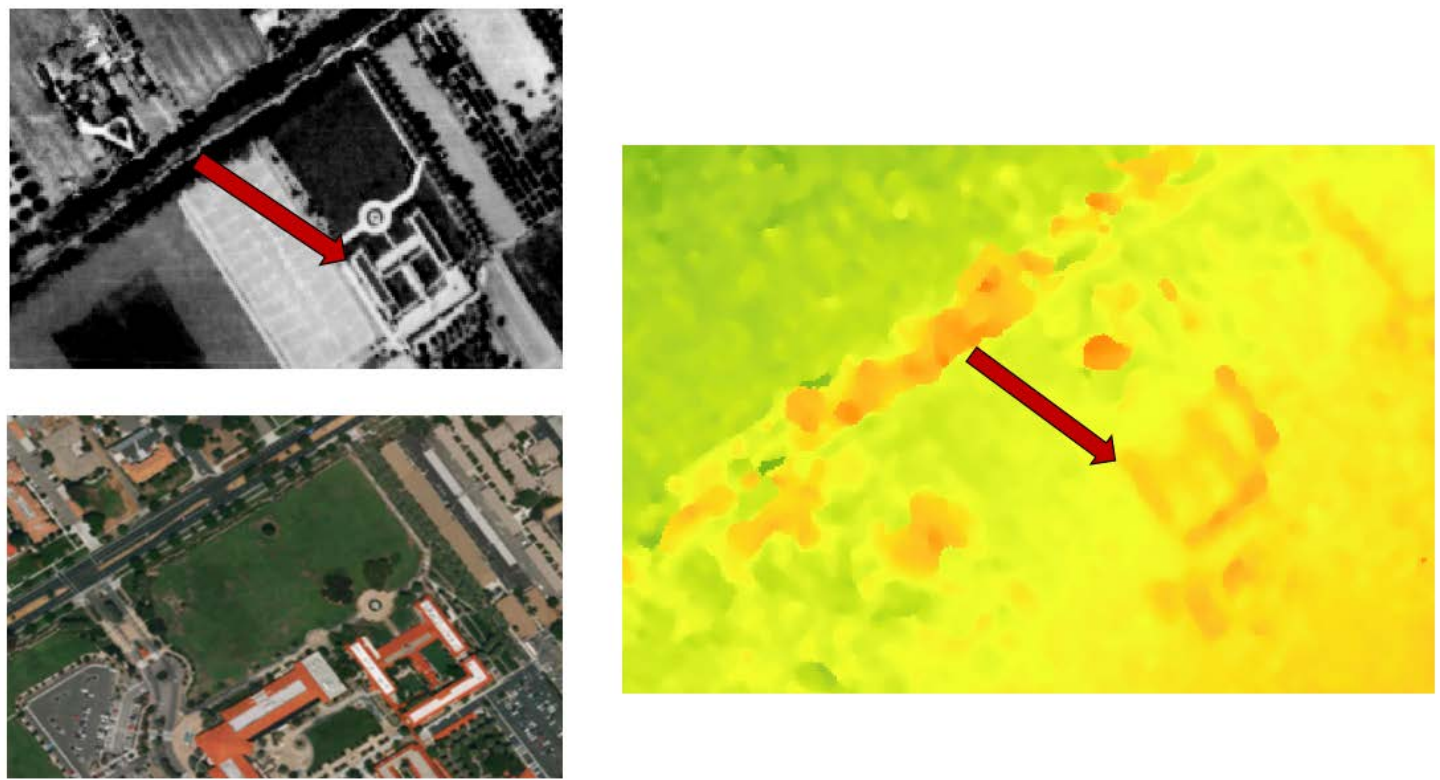

\section{Figure 6-17: California Baptist University}

One of the main buildings is clearly visible in the resulting DSM with an elevation of 7 to $10 \mathrm{~m}$ off the ground. It is impressive that the historical aerial film imagery could be used to extract the boundaries of individual buildings. In the imagery, it is also apparent that the adjacent buildings were not yet constructed at the time of the original survey, so this data could be used to aid in change detection over time. Other features like trees are also apparent in the imagery, proving the value of this type of analysis.

\subsubsection{Quarry}

A quarry was also visible in the project area and the site had experienced significant change since 1938. The quarry and a plot of elevation difference are shown in Figure 618.
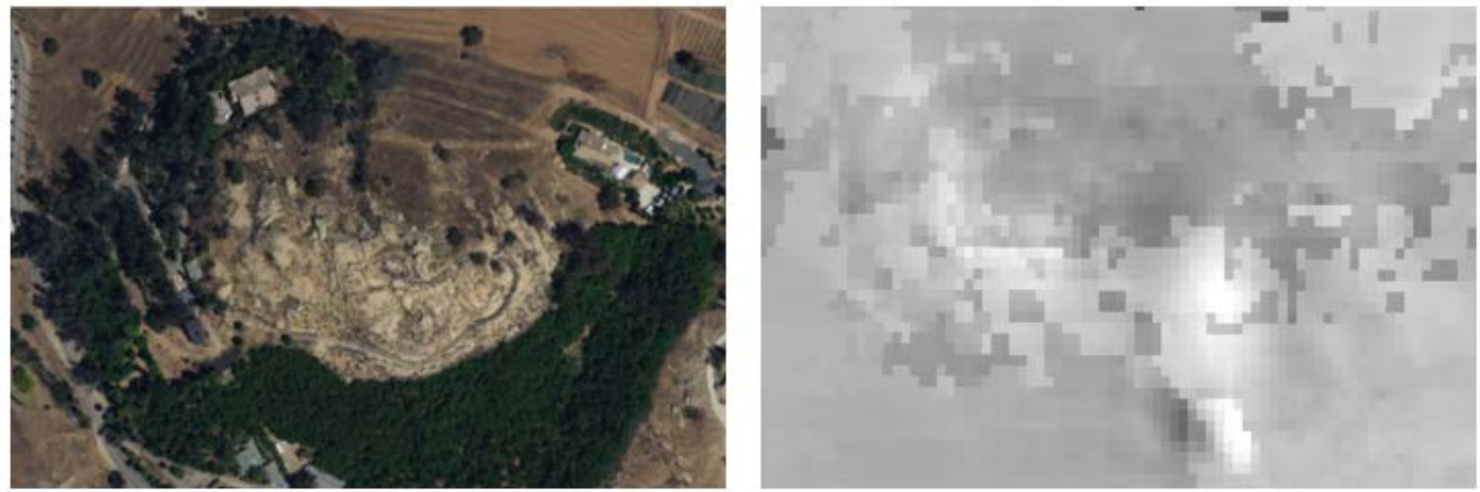

Figure 6-18: Quarry and elevation difference

The resulting DSM was noisy over the quarry, but around the quarry there was an approximate average difference of $9 \mathrm{~m}$ in elevation and over the quarry itself that 
increased to $16 \mathrm{~m}$. This indicated an approximate $7 \mathrm{~m}$ average elevation reduction over the quarry site over 80 years.

\subsubsection{Lake Mathews}

The principle feature of interest in the project site was Lake Mathews, which had not been completed and filled at the time of the 1938 aerial survey. This meant that the historical images over the site provided a valuable historical baseline that could be used as a comparison tool to evaluate how the countryside had changed over time. This project was able to extract a detailed DSM of the area, shown in Figure 6-19.

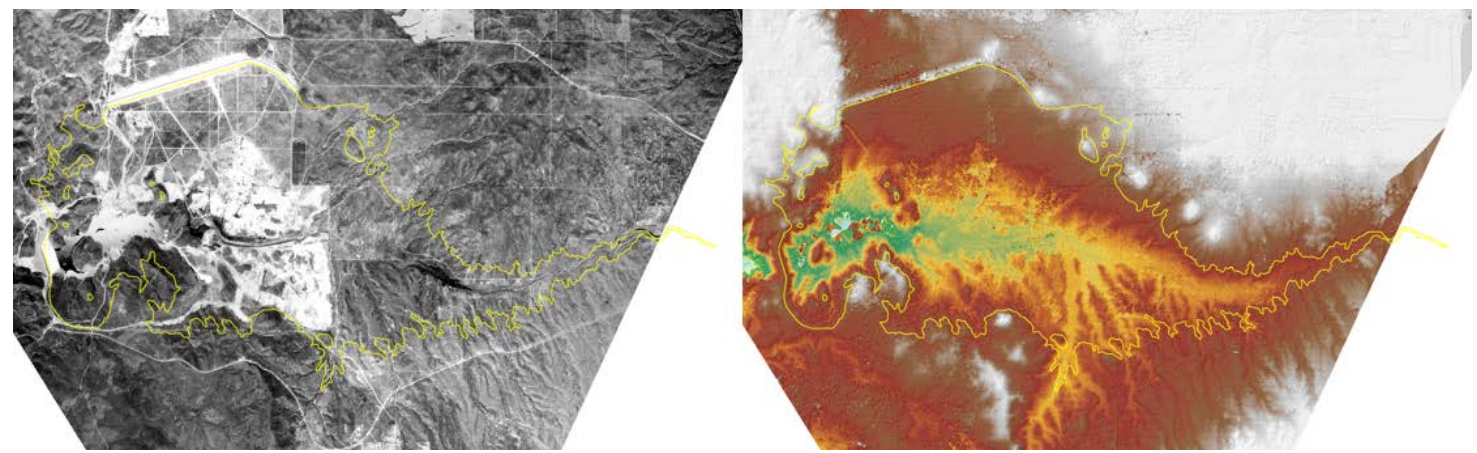

Figure 6-19: Lake Mathews orthomosaic and DSM with hillshade

The DSM could be used in environmental analysis to evaluate changes in drainage patterns for the area now that the area around the lake is fully developed. Being able to extract a feature of significance like this was one of the major exciting outcomes from this project.

\subsection{Processing Duration}

One of the goals of this project was to create a process that could be completed in a reasonable amount of time with minimal human interaction. The CCB reported that the processing for an image using the Buchwald method took approximately 30 to 45 minutes of manual work per image. For this project, the resulting processing time was drastically less assuming two conditions: many images are processed at one time and the only product required is an orthomosaic. When broken down on a per image basis there are economies of scale as shown in Table 5 below. Very few activities occur on a per image basis in this project and instead are performed at an area level which drove down the time spent on each individual image. The results are tabulated for the 28 images used for this project area. Additionally, just processing an orthomosaic would mean skipping a lot of the solution refinement process in Pix 4D shown below as an indeterminate amount of time. It is listed as indeterminate because it is impossible to accurately quantify. It is heavily dependent on the project area, the amount of overlap, the uncertainty in the control points and is a task that does not have a firm series of steps to lead specifically to completion as often different strategies need to be evaluated depending on the problems observed. 
Table 5. Estimated Durations for Project Stages Per Image

\begin{tabular}{|l|c|c|}
\hline \multicolumn{1}{|c|}{ Process } & $\begin{array}{c}\text { Manual Interaction } \\
\text { (minutes/image) }\end{array}$ & Difficulty \\
\hline Image standardization & 2 minutes & Low \\
\hline Determining image center & 2 minutes & Low \\
\hline Finding reference points & 5 minutes & Medium \\
\hline $\begin{array}{l}\text { Creating and running Pix4D } \\
\text { Processing session }\end{array}$ & 2 minutes & Low \\
\hline Tagging ground control in Pix 4D & 5 minutes & Medium \\
\hline Arcmap Georeferencing & 2 minutes & Low \\
\hline Refining solution in Pix4D & $\infty$ & Hard \\
\hline Using Manual Tie Points & 5 -10 minutes & Medium \\
\hline DSM clean-up in ArcGIS Pro & 5 minutes & Medium \\
\hline
\end{tabular}

In general, the Pix4D method should be considerably quicker and require less training depending on the project requirements. It puts a lot of emphasis on using computing power rather than manual effort to generate products. Each complete processing session including a regeneration of the orthorectified mosaic and DSM would take approximately three hours of computer time. Reevaluating different ground control combinations, however only took 10 minutes.

Using manual tie points in processing to correct the elevation artifacts was difficult because it was both manually intensive to identify the areas that needed them and find a match point in the area, but every iteration of processing required a three-hour computer reprocessing. If more images were involved this would lengthen the process immensely. This process may not be required in areas without farm fields, but in areas where the artifacts occur it would be better to use an alternate method. Overall by using some of the lessons learned in this project, highly accurate results could be created quickly with some practice. 


\section{Chapter 7 - Conclusions and Future Work}

Historical aerial imagery provides a valuable geographic baseline for change management and analysis, but to use these images effectively they need to be spatially oriented in the world. This is a challenging process because often the required supporting information about the camera or flight acquisition parameters are not present. This lack of supporting information limits the available processing techniques to ones that are manually time consuming, slow and costly to perform. Many organizations like the Center for Conservation of Biology at the University of California have large collections of historical aerial imagery but no staff with the time or specialized training to process them.

The goal of this project was to evaluate whether computer vision and photogrammetric processing technology could be used to process these image collections to a desired accuracy with less manual interaction and with more data products created, such as an elevation surface. To address the project goals and the issues associated with using historical aerial imagery, a semi-automated triangulation approach using the overlapping images together in series was selected and the processing was performed in Pix4D Professional.

A Python script was written to standardize the images to a consistent size and orientation to ensure all corrections applied to the images would be applied uniformly. The image centers were correlated to the ground using basemap data, and reference points found across the project site to be used as control and validation in processing. Pix4D Professional was used to process the imagery, which found keypoints in each image, matched them across multiple images and triangulated their position. After initial processing the ground control points were applied to the solution to increase positioning accuracy and the results were checked. Final adjustments to the output products and additional mapping and analysis were performed in ArcGIS Pro.

This solution successfully generated a seamless orthomosaic and DSM of the project area and these products can be used in subsequent analysis. Using an alternate method of georeferencing the final orthomosaic, the horizontal accuracy did meet the National Map Accuracy Standards, but in cases where only Pix4D Professional was used it could not reliably. This was primarily attributed to the large amounts of uncertainty in the control points used to control the accuracy of the solution and the difficulty finding suitably positioned ground control points across the project area. Nonetheless the results that were obtained were visually striking and identified many key features of interest that demonstrated the value of this technique in many forms of analysis depending on their scale. The processing workflow was faster than traditional methods due to the economies of scale of being able to process multiple images at once particularly when only an orthomosaic is required. The requirements for this project were met with caveats, this method may not produce the desired accuracy results in certain environmental situations some of which presented themselves in this project area.

\subsection{Future Work}

This project was a success but could be extended further to provide more benefit to improving the processing workflow and the quality of the output products. The first way 
this could be achieved is by improving the process of reducing or eliminating the elevation artifacts generated by Pix4D's matching process. The tools inside Pix4D require too much manual interaction. A proof of concept process was developed in ArcGIS Pro using the Raster Calculator to find and address the issues as part of this project, but it could be extended into a script that interpolated the missing elevation rather than replace it, and that also excluded performing a correction in certain user defined areas like over Lake Mathews. This revised DSM could then be used by Pix4D to reprocess the image mosaic for the project site and would correct the obvious artifacts in the image mosaic and the DSM.

Other opportunities for process improvement would lie in the Python standardization script. Currently the script requires users to manually select the fiducial marks in each image, but the fiducial marks are distinct enough that the script could be extended to find each fiducial mark automatically. This would save a significant amount of manual effort, particularly for large collections of imagery. Additionally, the process to determine the image centers could be improved by making a script to estimate the locations based on finding only the image centers at the end and middle of each flight line. The tool could then estimate the location of each image center using the project acquisition specifications which would be a helpful reference when trying to refine the image center further.

Geographic spatial analysis of the products over the project area was not included in the requirements for this project and would also be a significant opportunity for future work. The project site could be classified in 1938 for urban and rural areas and compared to present day to create maps indicating change. The site could be evaluated for changes in volume over time as well, and Lake Mathews could be evaluated for changes in drainage since construction. Now that the data can be processed in an efficient manner many spatial questions could be answered. Lastly the CCB has many years of historical aerial imagery, much of which overlaps the same terrain. Rather than comparing 80 years ago to present day it would be possible to process an area over multiple years and evaluate the changes over the terrain gradually. 


\section{Works Cited}

Barazzeti, L., Brumana, R., Oreni, D., Preyitali, M., \& Roncoroni, F. (2014 ). Trueorthophoto generation from UAV images: implementation of a combined photogrammetric and computer vision approach. ISPRS Annals of the Photogrammetry, Remote Sensing and Spatial Information Sciences, II(5), 57-63.

Brown, M., \& Lowe, D. (2003). Recognizing panoramas. Ninth International Conference on Computer Vision II, 1218-1225.

Buchwald, A. (2011). A production method for conversion of scanned historic aerial imagery into orthophotos using the rational function model. Redlands: University of Redlands.

Burns, J. H., \& Delparte, D. (2017). Comparison of commercial software structure from motion photogrammetry software used for underwater three-dimensional modelling of coral reef environments. Photogrammetry, Remote Sensing and Spatial Information Sciences, XLII, 127-132.

Eisenbeib, H. (2009). UAV Photogrammetry. E.T.H. Zurich.

Elevation. (2017, May 22). Retrieved June 5, 2017, from USGS - Science for a changing world: https://nationalmap.gov/elevation.html

Estes, J. E., Jensen, J. R., \& Tinney, L. R. (1977). The use of historical photography for mapping archaeological sites. Journal of Field Archaeology, 441-447.

FEMA. (2007). National Elevation Dataset. Retrieved from Federal Emergency Management Agency:

https://hazards.fema.gov/femaportal/docs/National\%20Elevation\%20Dataset_200 7.pdf

Garcia-Gago, J., Gonzalez-Aguilera, D., Gomez-Lahoz, J., \& San Jose-Alonso, I. (2014, 6(6)). A photogrammetric and computer vision-based approach for automated 3D architectural modeling and its typological analysis. Remote Sensing, 5671-5691.

Goncalves, J. A. (2016). Automatic orientation and mosaicing of archived aerial photography using structure from motion. Remote Sensing and Spatial Information Sciences, XL, 123-126.

Hexagon. (2016). Scanning Aerial Photography. Retrieved from Hexagon: https://hexagongeospatial.fluidtopics.net/reader/ P7L4c0T_d3papuwS98oGQ/fI0 M5LleRjUO0UxBveus6w

Jain, R., Kasturi, R., \& Schunck, B. G. (1995). Calibration. In R. Jain, R. Kasturi, \& B. G. Schunck, Machine Vision (pp. 309-310). New York: McGraw-Hill.

Kim, T., \& Muller, J.-P. (1996). Automated urban area building extraction from high resolution stereo imagery. Image and Vision Computing, 115-130.

Mayer, H. (2008). Object extraction in photogrammetric computer vision. ISPRS Journal of Photogrammetry and Remote Sensing, 213-222.

Monmonier, M. (2002, December). Aerial photography at the agricultural adjustment administration: acreage controls, conservation benefits and overhead surveillance in the 1930's. Retrieved from ASPRS: https://www.asprs.org/wpcontent/uploads/pers/2002journal/december/2002_dec_1257-1262.pdf

Monmonier, M. (2002). Aerial photography at the agricultural adjustment administration: acreage controls, conservations benefits, and overhead surveillance in the 1930s. Photogrammetric Engineering and Remote Sensing, 1257-1261. 
Morgan, J. L., Gergel, S. E., \& Coops, C. N. (2010). Aerial photography: a rapidly evolving tool for ecological management. BioScience, 48.

Morgan, J. L., Gergel, S. E., Ankerson, C., Tomscha, S. A., \& Sutherland, I. J. (2017). Historical aerial photography for landscape analysis. In J. L. Morgan, S. E. Gergel, C. Ankerson, S. A. Tomscha, \& I. J. Sutherland, Learning landscape ecology (pp. 22-23). New York: Springer-Verlag.

Nister, D. (2004). Transactions on pattern analysis and machine intelligence. IEEE Transactions on Pattern Analysis and Machine Intelligence, 756-770.

Pollefeys, M., Van Gool, I., Vergauwen, M., Verbiest, F., \& Cornelis, K. (2004). Visual modeling with a hand-held camera. International Journal of Computer Vision 59 (3), 207-232.

Remondino, F., Del Pizzo, S., Kersten, P. T., \& Troisi, S. (2012). Low-cost and open source solution for automated image orientation - a critical overview. EuroMed 2012: Progress in Cultural Heritage Preservation. 7616, pp. 40-54. Limassol, Cyprus: LNCS.

Stichelbaut, B. (2006). The application of first world war aerial photography to archaeology: the Belgian images. Antiquity, 80, 161-172.

Szeliski, R. (2011). Computer vision. Algorithms and applications, texts in computer science. New York: Springer.

Trucco, E., \& Verri, A. (1998). Introductory techniques for 3-D computer vision. New Jersey: Prentice Hall.

University of California. (2012). Imagery Report: AXM-1938A. Retrieved from UC Santa Barbara Library: http://mil.library.ucsb.edu/apcatalog/report/report.php?filed_by=AXM-1938A

USGS - Longterm Archive. (2015, January). Retrieved from High Resolution Orthoimagery: https://lta.cr.usgs.gov/high_res_ortho

Verhoeven, G. J. (2014). Producing orthophotophotos from archaelogical aerial photographs using computer vision. Profil, VIII, 13-17.

Verhoeven, G., Doneus, M., Briese, C., \& Vermeulen, F. (2012). Mapping by matching: a computer vision-based approach to fast and accurate georeferencing of archaelogical aerial photographs. Journal of Archaeological Science, 2060-2070.

Verhoeven, G., Sevara, C., Karel, W., Ressel, C., Doneus, M., \& Briese, C. (2013). Undistorting the past: new techniques for orthorectification of archaeological aerial frame imagery. In G. A. Wagner, C. E. Miller, \& H. Schutkowski, Natural Science in Archaeology (pp. 31-64). Switzerland: Springer International Publishing.

Visockiene, J. S., Brucas, D., \& Ragauskas, U. (2014). Comparison of UAV image processing softwares. Journal of Measurements in Engineering, II(2), 111-121.

Wolf, P. R., \& Dewitt, B. A. (2000). Cameras and other imaging devices. In P. R. Wolf, \& B. A. Dewitt, Elements of Photogrammetry (pp. 64-68, 136, 189-190). McGraw-Hill.

Ye Seul, L., Phu Hien, L., Soo, J., Mi Hee, L., Mu Wook, P., \& Jee-in , K. (2015). Calculation of tree height and canopy crown from drone images using segmentation. Journal of Korean Society of Surveying, Geodesy, Photogrammetry and Cartography, 33(6), 605-613. 


\section{Appendix A. Standardization Script Analysis}

Uncorrected images before standardization

\begin{tabular}{|c|c|c|c|c|c|}
\hline & \multicolumn{2}{|c|}{$\begin{array}{l}\text { Image Center } \\
\text { Coordinates }\end{array}$} & \multicolumn{2}{|c|}{$\begin{array}{c}\text { Photo Center } \\
\text { Coordinates }\end{array}$} & \multirow[b]{2}{*}{ Difference (pixels) } \\
\hline & $x$ & $\mathrm{Y}$ & $x$ & $Y$ & \\
\hline RE_AXM_035_006.tif & 4307 & 5467 & 4341 & 5490 & 41 \\
\hline RE_AXM_035_007.tif & 4330 & 5495 & 4366 & 5541 & 58 \\
\hline RE_AXM_035_008.tif & 4316 & 5502 & 4353 & 5496 & 37 \\
\hline RE_AXM_035_009.tif & 4362 & 5493 & 4380 & 5509 & 24 \\
\hline RE_AXM_035_081.tif & 4388 & 5469 & 4392 & 5464 & 6 \\
\hline RE_AXM_035_082.tif & 4367 & 5496 & 4392 & 5528 & 41 \\
\hline RE_AXM_035_083.tif & 4359 & 5474 & 4372 & 5509 & 38 \\
\hline RE_AXM_035_084.tif & 4376 & 5515 & 4347 & 5509 & 30 \\
\hline RE_AXM_035_085.tif & 4354 & 5471 & 4366 & 5464 & 15 \\
\hline RE_AXM_035_086.tif & 4363 & 5493 & 4386 & 5502 & 25 \\
\hline RE_AXM_039_073.tif & 4374 & 5464 & 4360 & 5477 & 19 \\
\hline RE_AXM_039_074.tif & 4387 & 5495 & 4379 & 5502 & 11 \\
\hline RE_AXM_039_083.tif & 4381 & 5437 & 4341 & 5451 & 43 \\
\hline RE_AXM_039_084.tif & 4379 & 5476 & 4380 & 5502 & 26 \\
\hline RE_AXM_039_085.tif & 4369 & 5508 & 4354 & 5515 & 17 \\
\hline RE_AXM_039_086.tif & 4413 & 5533 & 4405 & 5528 & 10 \\
\hline RE_AXM_039_087.tif & 4419 & 5472 & 4399 & 5502 & 37 \\
\hline RE_AXM_039_089.tif & 4390 & 5464 & 4366 & 5490 & 35 \\
\hline RE_AXM_040_033.tif & 4375 & 5491 & 4366 & 5496 & 10 \\
\hline RE_AXM_040_034.tif & 4365 & 5550 & 4366 & 5490 & 61 \\
\hline RE_AXM_040_036.tif & 4407 & 5463 & 4392 & 5490 & 30 \\
\hline RE_AXM_040_037.tif & 4368 & 5461 & 4284 & 5451 & 85 \\
\hline RE_AXM_040_038.tif & 4380 & 5475 & 4341 & 5464 & 41 \\
\hline RE_AXM_040_039.tif & 4397 & 5486 & 4347 & 5439 & 69 \\
\hline RE_AXM_040_040.tif & 4395 & 5450 & 4379 & 5477 & 31 \\
\hline RE_AXM_045_070.tif & 4364 & 5552 & 4379 & 5541 & 19 \\
\hline RE_AXM_045_071.tif & 4372 & 5485 & 4392 & 5541 & 59 \\
\hline RE_AXM_045_072.tif & 4378 & 5482 & 4379 & 5528 & 46 \\
\hline RE_AXM_045_073.tif & 4391 & 5460 & 4379 & 5502 & 44 \\
\hline RE_AXM_045_074.tif & 4378 & 5458 & 4405 & 5502 & 51 \\
\hline & & & & Average & 35 \\
\hline
\end{tabular}


Corrected images after standardization

\begin{tabular}{|c|c|c|c|c|c|}
\hline & \multicolumn{2}{|c|}{ Image Center Coordinates } & \multicolumn{2}{|c|}{$\begin{array}{l}\text { Photo Center } \\
\text { Coordinates }\end{array}$} & \multirow{2}{*}{$\begin{array}{c}\text { Difference } \\
\text { (pixels) }\end{array}$} \\
\hline & $X$ & $Y$ & $X$ & $Y$ & \\
\hline RE_AXM_035_006.tif & 4250 & 5422 & 4252 & 5423 & 1.8 \\
\hline RE_AXM_035_007.tif & 4250 & 5422 & 4252 & 5423 & 2.0 \\
\hline RE_AXM_035_008.tif & 4250 & 5422 & 4251 & 5422 & 0.5 \\
\hline RE_AXM_035_009.tif & 4250 & 5422 & 4250 & 5422 & 0.4 \\
\hline RE_AXM_035_081.tif & 4250 & 5422 & 4250 & 5422 & 0.3 \\
\hline RE_AXM_035_082.tif & 4250 & 5422 & 4251 & 5423 & 1.8 \\
\hline RE_AXM_035_083.tif & 4250 & 5422 & 4251 & 5422 & 1.3 \\
\hline RE_AXM_035_084.tif & 4250 & 5422 & 4250 & 5423 & 1.0 \\
\hline RE_AXM_035_085.tif & 4250 & 5422 & 4252 & 5421 & 1.9 \\
\hline RE_AXM_035_086.tif & 4250 & 5422 & 4249 & 5422 & 0.8 \\
\hline RE_AXM_039_073.tif & 4250 & 5422 & 4250 & 5422 & 0.6 \\
\hline RE_AXM_039_074.tif & 4250 & 5422 & 4251 & 5421 & 1.8 \\
\hline RE_AXM_039_083.tif & 4250 & 5422 & 4251 & 5422 & 1.0 \\
\hline RE_AXM_039_084.tif & 4250 & 5422 & 4250 & 5423 & 0.8 \\
\hline RE_AXM_039_085.tif & 4250 & 5422 & 4251 & 5423 & 1.3 \\
\hline RE_AXM_039_086.tif & 4250 & 5422 & 4250 & 5422 & 0.3 \\
\hline RE_AXM_039_087.tif & 4250 & 5422 & 4250 & 5422 & 0.3 \\
\hline RE_AXM_039_089.tif & 4250 & 5422 & 4251 & 5422 & 1.3 \\
\hline RE_AXM_040_033.tif & 4250 & 5422 & 4251 & 5422 & 0.6 \\
\hline RE_AXM_040_034.tif & 4250 & 5422 & 4251 & 5421 & 1.5 \\
\hline RE_AXM_040_036.tif & 4250 & 5422 & 4251 & 5422 & 1.0 \\
\hline RE_AXM_040_037.tif & 4250 & 5422 & 4251 & 5422 & 1.0 \\
\hline RE_AXM_040_038.tif & 4250 & 5422 & 4250 & 5422 & 0.4 \\
\hline RE_AXM_040_039.tif & 4250 & 5422 & 4250 & 5422 & 0.4 \\
\hline RE_AXM_040_040.tif & 4250 & 5422 & 4251 & 5423 & 1.5 \\
\hline RE_AXM_045_070.tif & 4250 & 5422 & 4250 & 5423 & 1.3 \\
\hline RE_AXM_045_071.tif & 4250 & 5422 & 4252 & 5422 & 1.8 \\
\hline RE_AXM_045_072.tif & 4250 & 5422 & 4251 & 5423 & 0.9 \\
\hline RE_AXM_045_073.tif & 4250 & 5422 & 4251 & 5421 & 0.9 \\
\hline \multirow[t]{2}{*}{ RE_AXM_045_074.tif } & 4250 & 5422 & 4250 & 5422 & 0.3 \\
\hline & & & & Average & 1 \\
\hline
\end{tabular}




\section{Appendix B. Analysis and Quality Reports}

Overview of all reference points (black) and image centers (yellow)

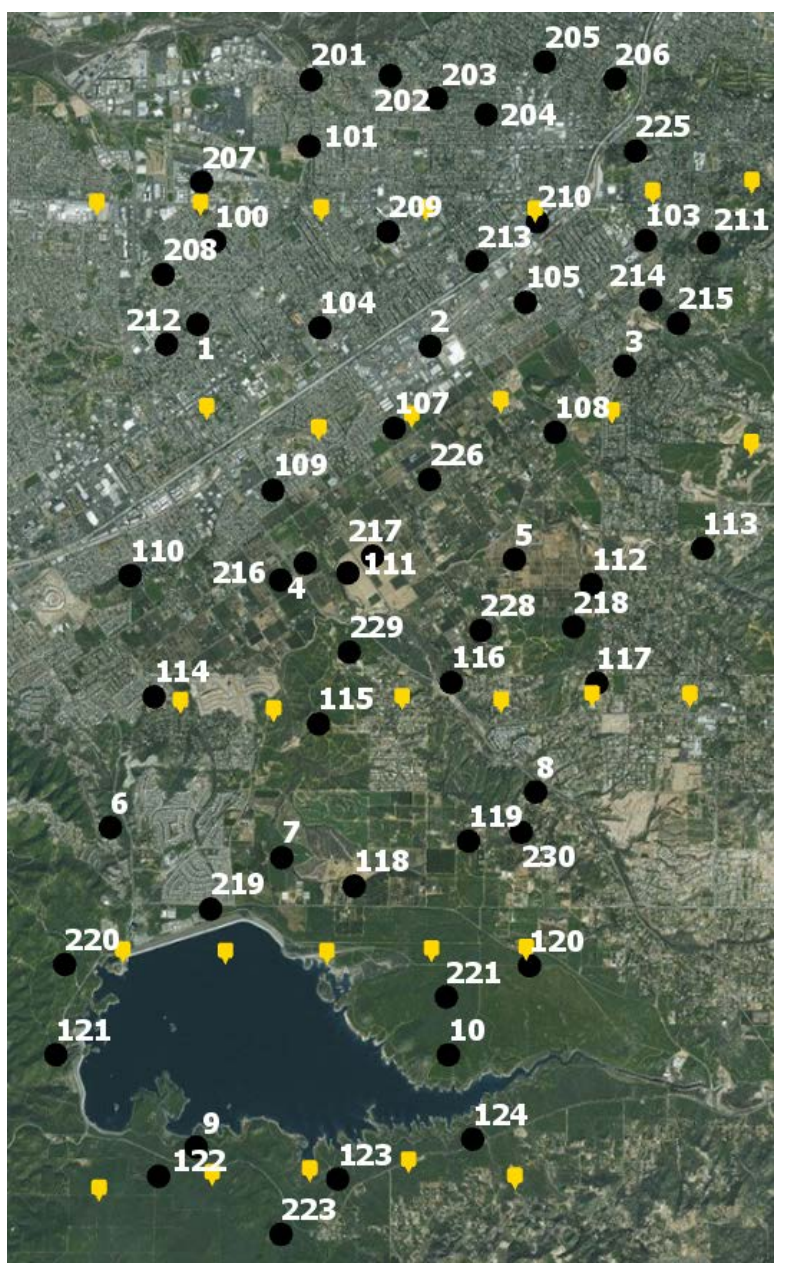


Results of georeferencing the Pix4D output mosaic

\begin{tabular}{cccccccc} 
& \multicolumn{2}{c}{ Reference } & \multicolumn{2}{c}{ Computed } & \multicolumn{2}{c}{ Residual } \\
& $X$ & $Y$ & $X$ & $Y$ & $X$ & $Y$ & RMSE \\
33 & 462163.4 & 3754267.1 & 462165.7 & 3754267.0 & -2.3 & 0.0 & 2.3 \\
204 & 461029.2 & 3751120.6 & 461023.2 & 3751124.4 & 6.0 & -3.8 & 7.1 \\
902 & 458940.1 & 3754574.1 & 458941.9 & 3754573.4 & -1.8 & 0.7 & 1.9 \\
202 & 464865.0 & 3753992.6 & 464865.1 & 3753994.2 & -0.1 & -1.6 & 1.6 \\
35 & 463334.7 & 3751310.4 & 463336.2 & 3751314.1 & -1.5 & -3.8 & 4.0 \\
21 & 463625.8 & 3748092.6 & 463629.6 & 3748087.7 & -3.8 & 4.9 & 6.2 \\
38 & 460108.3 & 3747176.2 & 460107.7 & 3747176.4 & 0.6 & -0.1 & 0.6 \\
106 & 457717.7 & 3747595.7 & 457727.2 & 3747593.6 & -9.5 & 2.0 & 9.7 \\
107 & 462414.2 & 3744442.3 & 462418.1 & 3744441.6 & -3.9 & 0.7 & 4.0 \\
900 & 458928.8 & 3743155.2 & 458919.2 & 3743163.5 & 9.6 & -8.3 & 12.7
\end{tabular}

$\begin{array}{cccccccc}\text { Check } & & & & & & \\ \text { Points } & & & & & & \\ 3 & 462612.3 & 3756863.8 & 462603.6 & 3756853.1 & 8.7 & 10.6 & 13.8 \\ 8 & 460637.4 & 3754525.3 & 460636.9 & 3754522.4 & 0.5 & 2.9 & 3.0 \\ 40 & 463488.7 & 3754877.3 & 463495.7 & 3754884.5 & -7.0 & -7.2 & 10.0 \\ 108 & 456971.1 & 3744442.8 & 456956.6 & 3744470.6 & 14.4 & -27.8 & 31.3 \\ 111 & 460739.3 & 3740786.5 & 460726.2 & 3740785.2 & 13.1 & 1.4 & 13.2 \\ 112 & 458396.3 & 3742757.4 & 458380.0 & 3742777.9 & 16.2 & -20.5 & 26.2 \\ 113 & 460882.6 & 3742718.0 & 460876.0 & 3742724.8 & 6.5 & -6.8 & 9.4 \\ 114 & 462754.6 & 3743273.7 & 462756.6 & 3743270.2 & -2.1 & 3.5 & 4.1 \\ 115 & 463543.3 & 3745677.8 & 463549.6 & 3745679.8 & -6.4 & -2.0 & 6.7 \\ 116 & 461111.2 & 3746781.9 & 461118.9 & 3746780.6 & -7.7 & 1.3 & 7.8 \\ 118 & 458332.7 & 3749407.1 & 458328.1 & 3749415.8 & 4.6 & -8.7 & 9.9 \\ 119 & 458001.7 & 3751090.0 & 457986.3 & 3751095.8 & 15.4 & -5.8 & 16.5 \\ 120 & 460616.4 & 3749030.0 & 460612.8 & 3749027.1 & 3.6 & 2.9 & 4.6 \\ 121 & 462456.1 & 3749606.2 & 462455.7 & 3749617.8 & 0.4 & -11.6 & 11.6 \\ 122 & 464479.3 & 3749597.3 & 464491.6 & 3749606.5 & -12.2 & -9.3 & 15.3 \\ 124 & 463900.2 & 3753074.3 & 463906.6 & 3753079.0 & -6.3 & -4.6 & 7.8 \\ 125 & 461666.6 & 3753135.2 & 461667.7 & 3753138.0 & -1.1 & -2.8 & 3.1 \\ 126 & 459983.8 & 3752271.6 & 459985.0 & 3752275.1 & -1.2 & -3.5 & 3.7 \\ 127 & 457887.0 & 3752876.2 & 457887.5 & 3752886.7 & -0.5 & -10.5 & 10.5 \\ 129 & 462814.2 & 3755447.9 & 462803.6 & 3755442.7 & 10.6 & 5.1 & 11.8 \\ 130 & 459176.4 & 3755724.1 & 459176.5 & 3755716.3 & -0.2 & 7.9 & 7.9 \\ 132 & 460487.3 & 3757042.3 & 460490.5 & 3757040.5 & -3.2 & 1.9 & 3.7 \\ 205 & 460430.8 & 3751258.3 & 460426.5 & 3751260.9 & 4.3 & -2.5 & 5.0 \\ 301 & 464401.0 & 3750964.2 & 464406.9 & 3750964.3 & -5.9 & -0.1 & 5.9\end{array}$


Result for processing session with primarily 2D control points and reduced 3D control
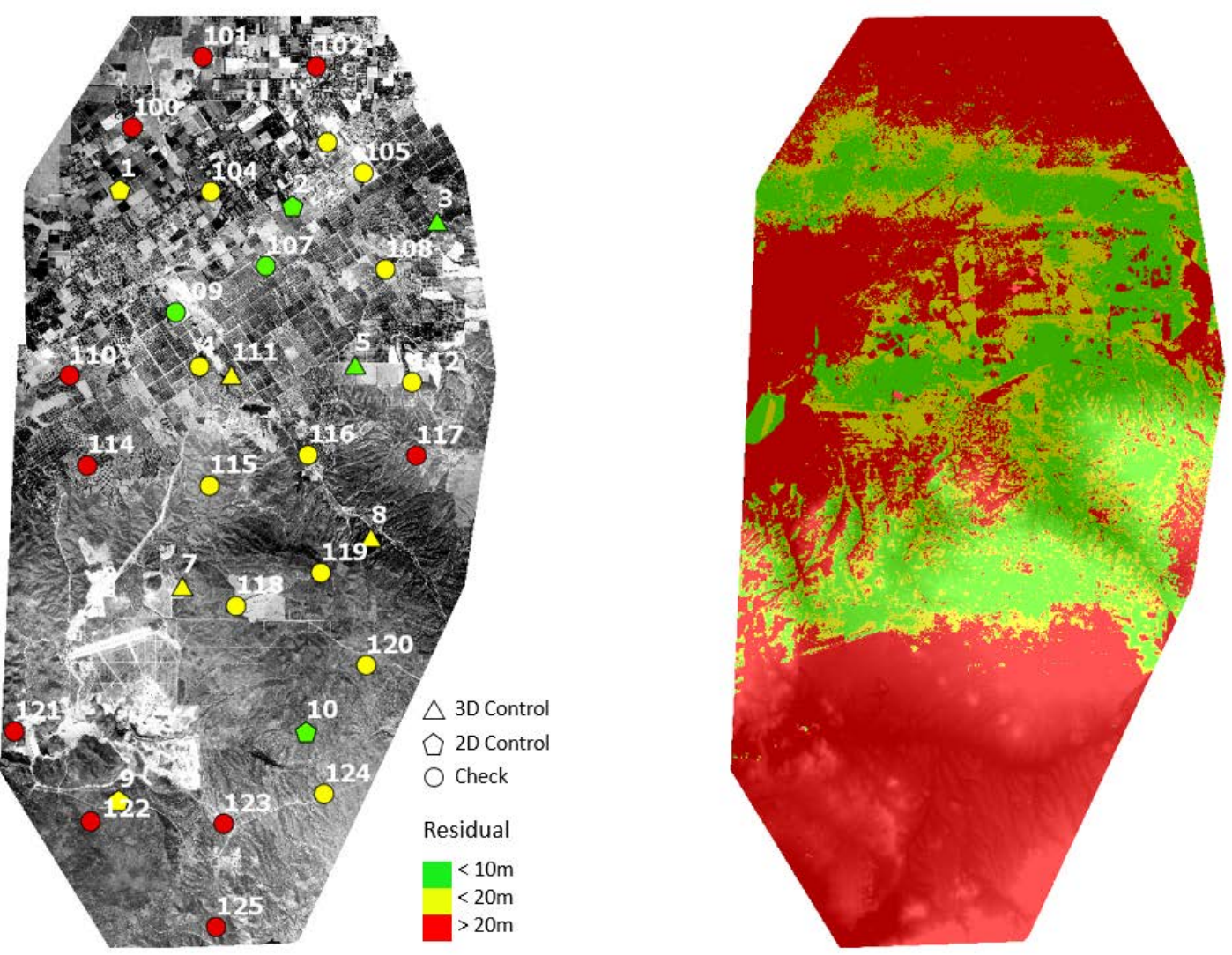
Pix4D Professional Quality Report for All Control processing session

\section{Quality Report}

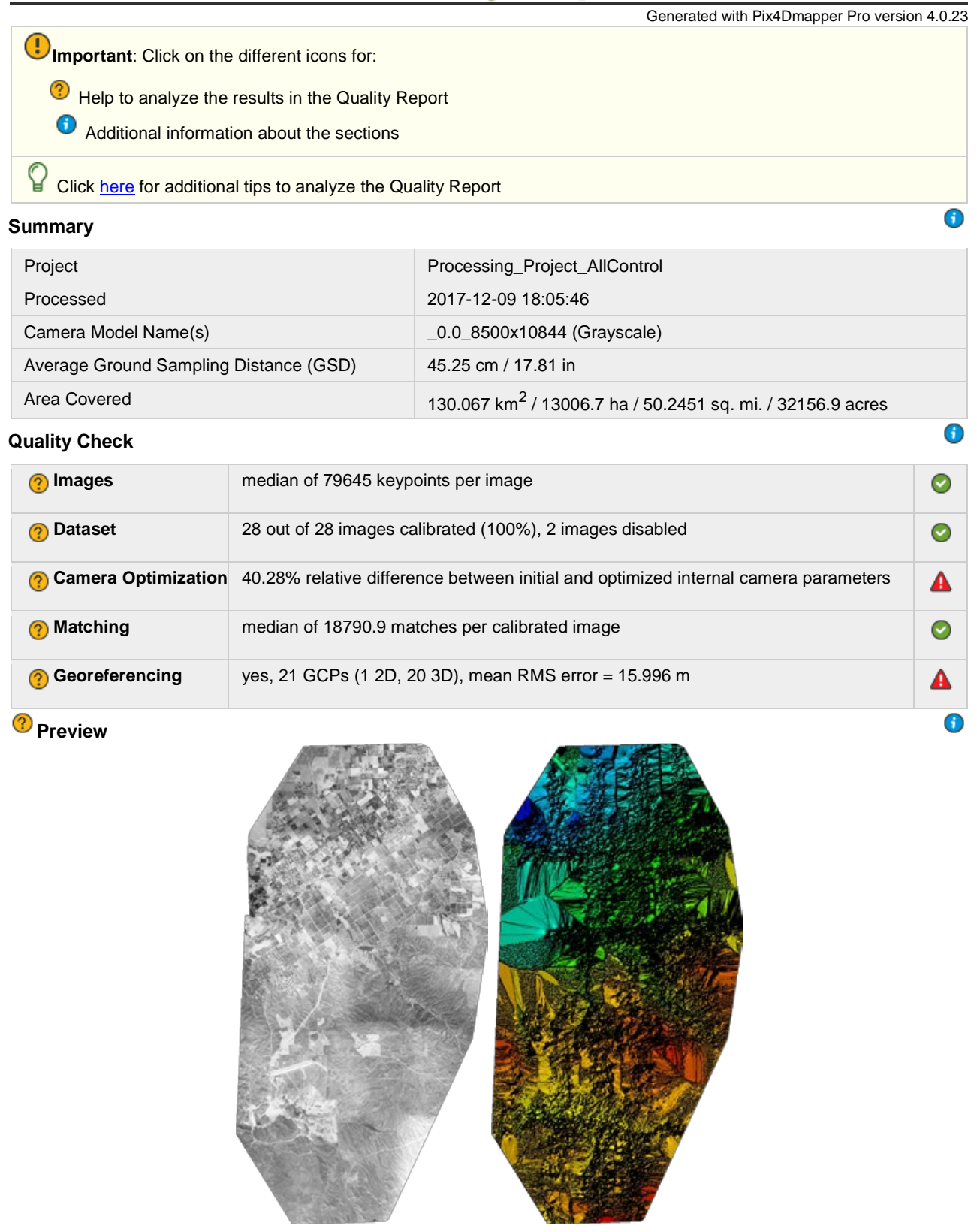

Figure 1: Orthomosaic and the corresponding sparse Digital Surface Model (DSM) before densification.

\section{Calibration Details}




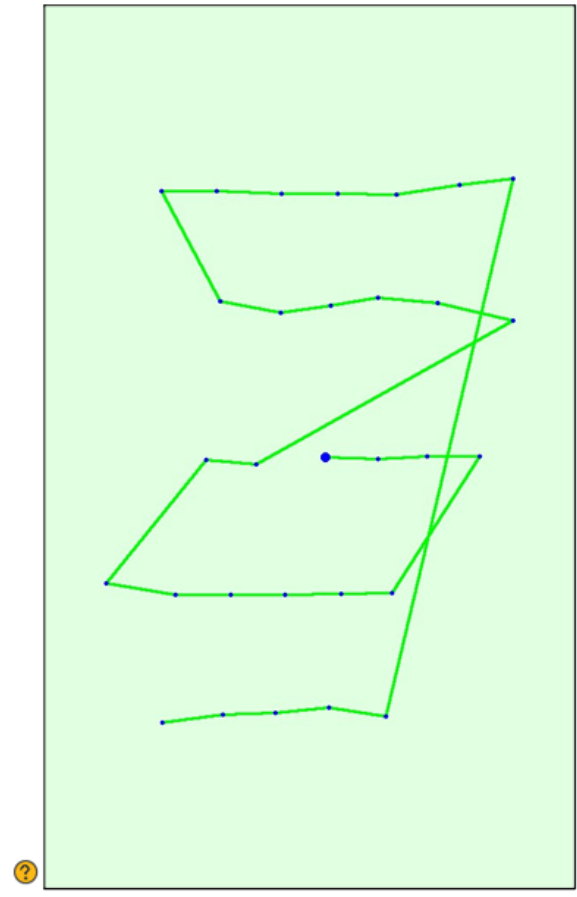

Figure 2: Top view of the initial image position. The green line follows the position of the images in time starting from the large blue dot.

(3) Computed Image/GCPs/Manual Tie Points Positions

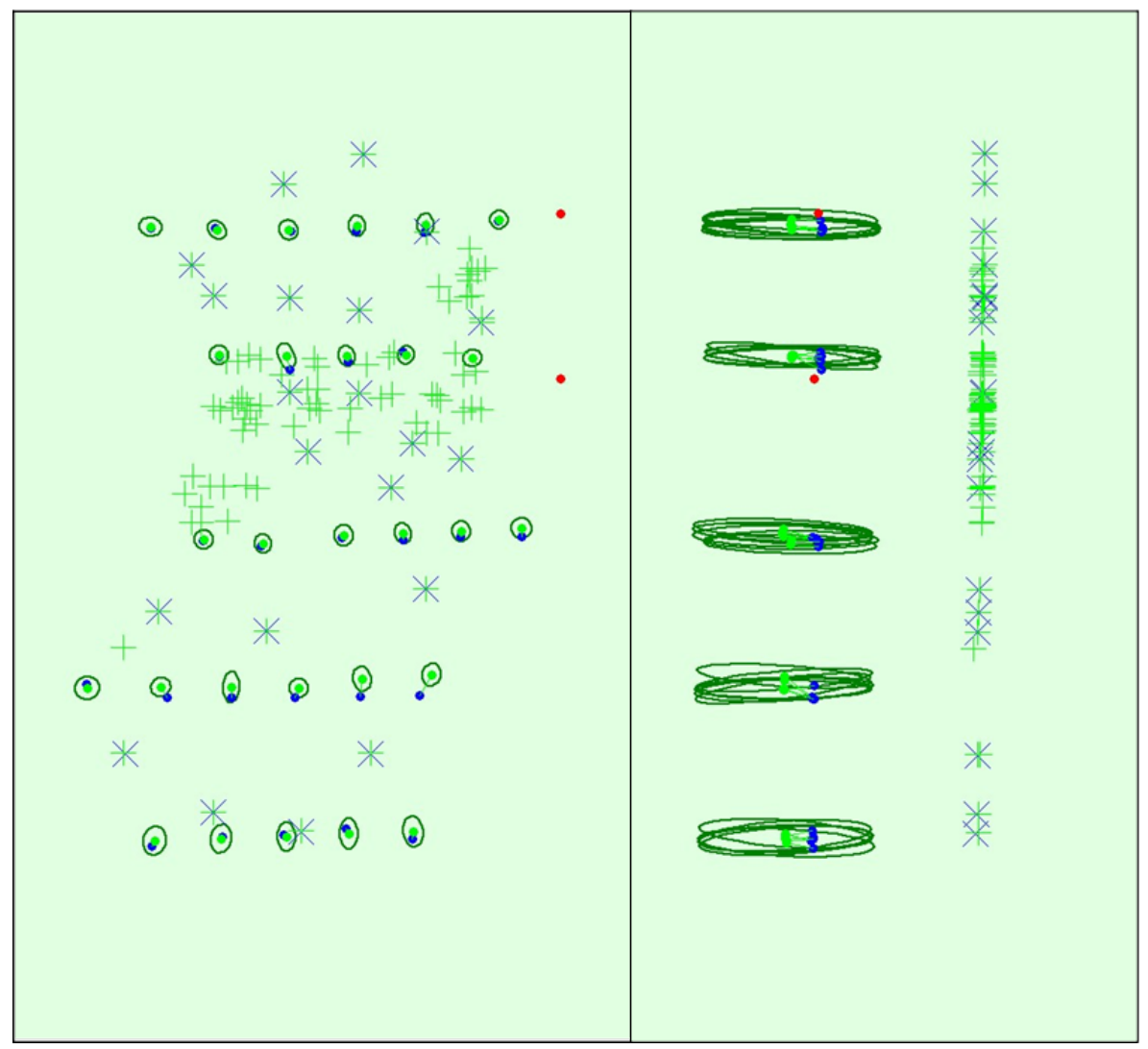




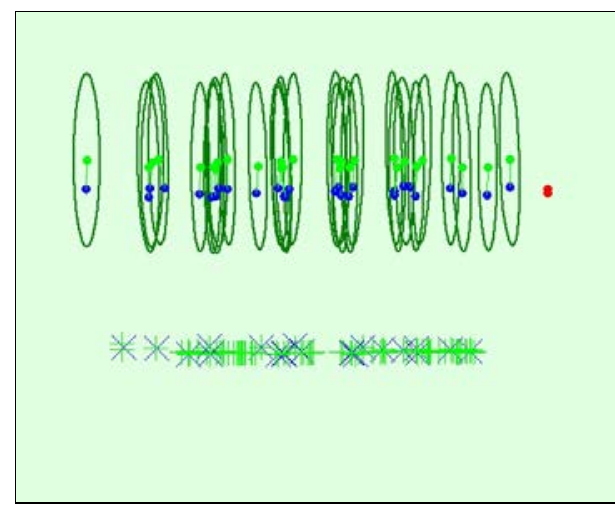

Uncertainty ellipses $100 x$ magnified

Figure 3: Offset between initial (blue dots) and computed (green dots) image positions as well as the offset between the GCPs initial positions (blue crosses) and their computed positions (green crosses) in the top-view (XY plane), front-view ( $X Z$ plane), and side-view
( $Y Z$ plane). Red dots indicate disabled or uncalibrated images. Dark green ellipses indicate the absolute position uncertainty of the
bundle block adjustment result.

(?) Absolute camera position and orientation uncertainties

(1)

\begin{tabular}{|l|l|l|l|l|l|l|} 
& $\mathrm{X}[\mathrm{m}]$ & $\mathrm{Y}[\mathrm{m}]$ & $\mathrm{Z}[\mathrm{m}]$ & Omega [degree] & Phi [degree] & Kappa [degree] \\
\hline Mean & 2.131 & 2.503 & 19.382 & 0.017 & 0.016 & 0.006 \\
\hline Sigma & 0.226 & 0.456 & 0.244 & 0.008 & 0.004 & 0.002 \\
\hline
\end{tabular}

(3) Overlap

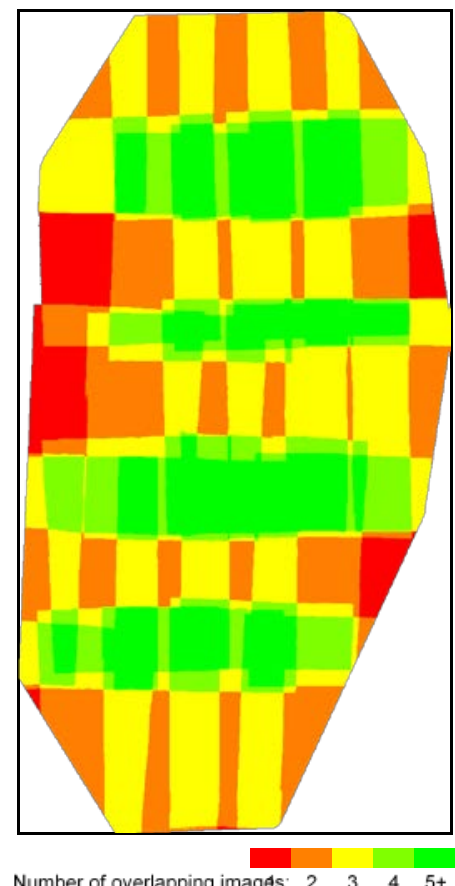

Figure 4: Number of overlapping images computed for each pixel of the orthomosaic. Red and yellow areas indicate low overlap for which poor results may be generated. Green areas indicate an overlap of over 5 images
for every pixel. Good quality results will be generated as long as the number of keypoint matches is also sufficient for these areas (see Figure 5 for keypoint matches).

\section{Bundle Block Adjustment Details}


(2) Internal Camera Parameters_0.0_8500x10844 (Grayscale). Sensor Dimensions: 25.400 [mm] x 32.404 [mm] (2)

EXIF ID:_0.0_8500x10844

\begin{tabular}{|c|c|c|c|c|c|c|c|c|}
\hline & $\begin{array}{l}\text { Focal } \\
\text { Length }\end{array}$ & $\begin{array}{l}\text { Principal } \\
\text { Point } x\end{array}$ & $\begin{array}{l}\text { Principal } \\
\text { Point } y\end{array}$ & R1 & R2 & R3 & $\mathrm{T} 1$ & $\mathrm{~T} 2$ \\
\hline Initial V alues & $\begin{array}{l}6692.914 \\
\text { [pixel] } \\
20.000[\mathrm{~mm}]\end{array}$ & $\begin{array}{l}4250.000 \\
\text { [pixel] } \\
12.700[\mathrm{~mm}]\end{array}$ & $\begin{array}{l}5422.001 \\
\text { [pixel] } \\
16.202[\mathrm{~mm}]\end{array}$ & 0.000 & 0.000 & 0.000 & 0.000 & 0.000 \\
\hline Optimized Values & $\begin{array}{l}9389.332 \\
\text { [pixel] } \\
28.058 \text { [mm] }\end{array}$ & $\begin{array}{l}4320.682 \\
\text { [pixel] } \\
12.911[\mathrm{~mm}]\end{array}$ & $\begin{array}{l}5362.897 \\
\text { [pixel] } \\
16.026[\mathrm{~mm}]\end{array}$ & 0.005 & $\overline{-} .003$ & 0.017 & $\overline{-} .001$ & 0.000 \\
\hline $\begin{array}{l}\text { Uncertainties } \\
\text { (Sigma) }\end{array}$ & $\begin{array}{l}42.920 \text { [pixel] } \\
0.128 \text { [mm] }\end{array}$ & $\begin{array}{l}4.207 \text { [pixel] } \\
0.013 \text { [mm] }\end{array}$ & $\begin{array}{l}4.558 \text { [pixel] } \\
0.014[\mathrm{~mm}]\end{array}$ & 0.000 & 0.002 & 0.003 & 0.000 & 0.000 \\
\hline
\end{tabular}

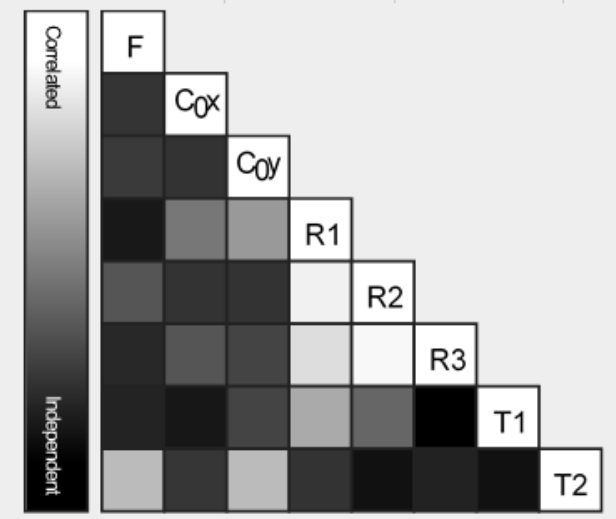

The correlation between camera internal parameters determined by the bundle adjustment. White indicates a full correlation between the parameters, ie. any change in one can be fully compensated by the other. Black indicates that the parameter is completely independent, and is not affected by other parameters.

The number of Automatic Tie Points (ATPs) per pixel, averaged over all images of the camera model, is color coded between black and white. White indicates that, on average, more than 16 ATPs have been extracted at the pixel location. Black indicates that, on average, O ATPs have been extracted at the pixel location. Click on the image to the see the average direction and magnitude of the reprojection error for each pixel. Note that the vectors are scaled for better visualization. The scale bar indicates the magnitude of 1 pixel error.

(?) 2D Keypoints Table

\begin{tabular}{|l|l|l|}
\hline & Number of 2D Keypoints per Image & Number of M atched 2D Keypoints per Image \\
\hline Median & 79645 & 18791 \\
\hline Min & 66668 & 8854 \\
\hline Max & 86825 & 27634 \\
\hline Mean & 79224 & 18639 \\
\hline
\end{tabular}

(3) Points from 2D Keypoint Matches

\begin{tabular}{|l|l|}
\hline & Number of 3D Points Observed \\
\hline In 2 Images & 208735 \\
\hline In 3 Images & 28320 \\
\hline In 4 Images & 3364 \\
\hline In 5 Images & 978 \\
\hline In 6 Images & 188 \\
\hline
\end{tabular}




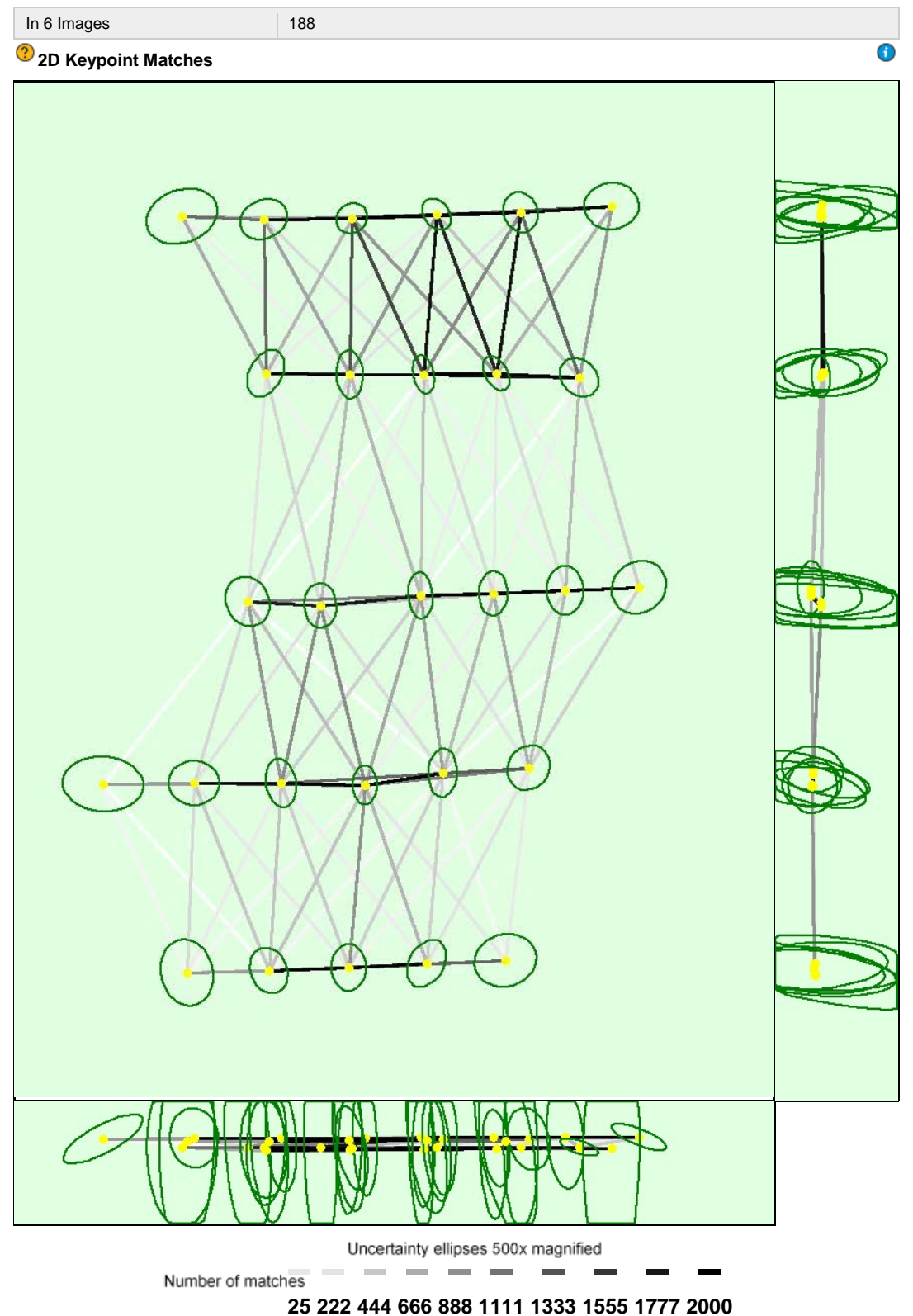

Figure 5: Computed image positions with links between matched images. The darkness of the links indicates the number of matched $2 D$ keypoints between the images. Bright links indicate weak links and require manual tie points or more images. Dark green ellipses
indicate the relative camera position uncertainty of the bundle block adjustment result.

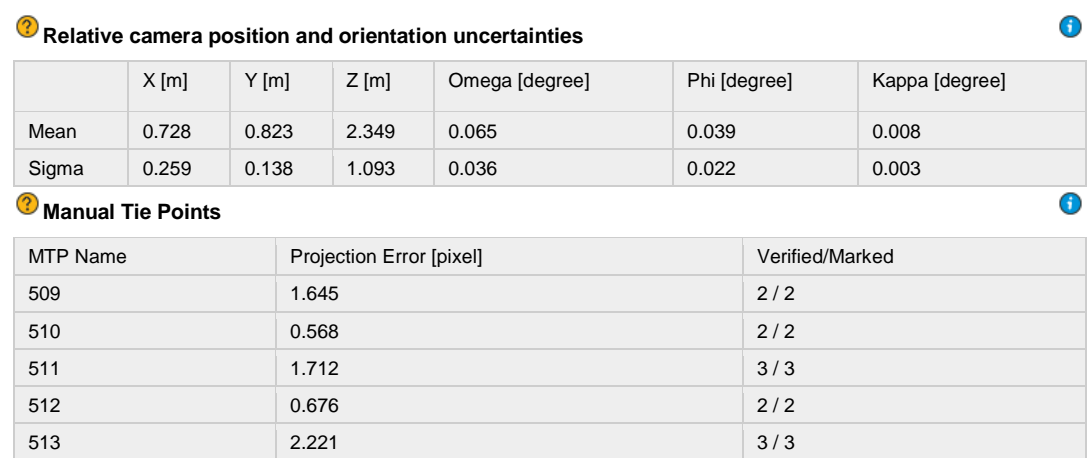




\begin{tabular}{|c|c|c|}
\hline mtp90 & 1.502 & $2 / 2$ \\
\hline mtp91 & 1.287 & $2 / 2$ \\
\hline mtp92 & 2.057 & $3 / 3$ \\
\hline mtp93 & 1.188 & $2 / 2$ \\
\hline mtp94 & 1.229 & $2 / 2$ \\
\hline mtp95 & 1.891 & $2 / 2$ \\
\hline mtp96 & 1.564 & $2 / 2$ \\
\hline 515 & 1.383 & $2 / 2$ \\
\hline 516 & 1.164 & $2 / 2$ \\
\hline 517 & 1.487 & $3 / 3$ \\
\hline 518 & 1.389 & $2 / 2$ \\
\hline 519 & 1.281 & $2 / 2$ \\
\hline 520 & 0.061 & $2 / 2$ \\
\hline 521 & 1.111 & $2 / 2$ \\
\hline 522 & 0.426 & $2 / 2$ \\
\hline 523 & 0.684 & $2 / 2$ \\
\hline 524 & 1.044 & $2 / 2$ \\
\hline 525 & 0.335 & $2 / 2$ \\
\hline 526 & 1.751 & $2 / 2$ \\
\hline 527 & 1.310 & $2 / 2$ \\
\hline 528 & 0.925 & $2 / 2$ \\
\hline 529 & 1.038 & $2 / 2$ \\
\hline 530 & 1.368 & $2 / 2$ \\
\hline 531 & 2.117 & $2 / 2$ \\
\hline 532 & 1.378 & $3 / 3$ \\
\hline 533 & 1.871 & $2 / 2$ \\
\hline 534 & 0.803 & $2 / 2$ \\
\hline 535 & 0.790 & $2 / 2$ \\
\hline 536 & 0.437 & $2 / 2$ \\
\hline 537 & 0.669 & $2 / 2$ \\
\hline 538 & 0.639 & $2 / 2$ \\
\hline 539 & 0.566 & $2 / 2$ \\
\hline 540 & 1.155 & $2 / 2$ \\
\hline 541 & 0.988 & $2 / 2$ \\
\hline 542 & 0.570 & $2 / 2$ \\
\hline 543 & 0.120 & $2 / 2$ \\
\hline 544 & 0.850 & $3 / 3$ \\
\hline 545 & 0.131 & $2 / 2$ \\
\hline 546 & 1.096 & $2 / 2$ \\
\hline 549 & 3.169 & $5 / 5$ \\
\hline 550 & 1.598 & $3 / 3$ \\
\hline 551 & 1.335 & $2 / 2$ \\
\hline 552 & 0.067 & $2 / 2$ \\
\hline 553 & 1.080 & $3 / 3$ \\
\hline 554 & 2.126 & $5 / 5$ \\
\hline 556 & 1.980 & $6 / 6$ \\
\hline 559 & 0.104 & $2 / 2$ \\
\hline 562 & 0.142 & $3 / 3$ \\
\hline 564 & 2.381 & $4 / 4$ \\
\hline 565 & 2.029 & $4 / 4$ \\
\hline 566 & 1.890 & $4 / 4$ \\
\hline 567 & 1.277 & $4 / 4$ \\
\hline 568 & 0.823 & $4 / 4$ \\
\hline 569 & 2.306 & $4 / 4$ \\
\hline 570 & 1.435 & $4 / 4$ \\
\hline 571 & 2.179 & $4 / 4$ \\
\hline 572 & 2.425 & $5 / 5$ \\
\hline 573 & 3.259 & $3 / 3$ \\
\hline
\end{tabular}


574

2.346

$4 / 4$

Projection errors for manual tie points. The last column counts the number of images where the manual tie point has been
automatically verified vs. manually marked.

Geolocation Details

(3) Ground Control Points

\begin{tabular}{|c|c|c|c|c|c|c|}
\hline GCP Name & $\begin{array}{l}\text { Accuracy XY/Z } \\
{[\mathrm{m}]}\end{array}$ & Error $X[\mathrm{~m}]$ & Error $\mathrm{Y}[\mathrm{m}]$ & Error Z [m] & $\begin{array}{l}\text { Projection Error } \\
\text { [pixel] }\end{array}$ & Verified/Marked \\
\hline 208 (3D) & $5.000 / 5.000$ & 13.208 & 6.805 & 4.069 & 2.254 & $3 / 3$ \\
\hline 104 (3D) & $5.000 / 5.000$ & 7.481 & 6.352 & -4.528 & 1.674 & $6 / 6$ \\
\hline 226 (3D) & $5.000 / 5.000$ & 4.211 & -0.810 & 0.853 & 2.147 & $3 / 3$ \\
\hline 227 (3D) & $5.000 / 5.000$ & 11.593 & -5.079 & -0.424 & 1.579 & $3 / 3$ \\
\hline 228 (3D) & $5.000 / 5.000$ & -10.126 & -6.095 & -2.599 & 1.479 & $3 / 3$ \\
\hline $8(3 D)$ & $5.000 / 5.000$ & -12.604 & -6.561 & 6.225 & 1.889 & $5 / 5$ \\
\hline 203 (3D) & $5.000 / 5.000$ & -3.317 & 19.291 & 5.672 & 1.632 & $3 / 3$ \\
\hline $6(3 \mathrm{D})$ & $5.000 / 5.000$ & 23.717 & -13.350 & 0.132 & 1.408 & $4 / 4$ \\
\hline $10(2 \mathrm{D})$ & $5.000 / 5.000$ & -7.172 & -17.560 & & 2.323 & $5 / 5$ \\
\hline $121(3 \mathrm{D})$ & $5.000 / 5.000$ & 40.705 & -38.009 & 40.647 & 1.258 & $3 / 3$ \\
\hline $123(3 \mathrm{D})$ & $5.000 / 5.000$ & 10.211 & -33.013 & 60.288 & 0.152 & $3 / 3$ \\
\hline $210(3 \mathrm{D})$ & $5.000 / 5.000$ & -17.808 & 7.476 & -1.579 & 0.731 & $3 / 3$ \\
\hline $101(3 \mathrm{D})$ & $5.000 / 5.000$ & 5.767 & 9.063 & -1.316 & 2.104 & $3 / 3$ \\
\hline $2(3 \mathrm{D})$ & $5.000 / 5.000$ & 0.191 & 3.364 & -1.385 & 2.018 & $6 / 6$ \\
\hline $5(3 D)$ & $5.000 / 5.000$ & -12.368 & -1.555 & 6.299 & 2.407 & $5 / 5$ \\
\hline 7 (3D) & $5.000 / 5.000$ & 9.802 & -0.406 & 4.414 & 2.224 & $5 / 5$ \\
\hline $3(3 \mathrm{D})$ & $5.000 / 5.000$ & -23.299 & 5.788 & -4.280 & 2.309 & $4 / 4$ \\
\hline 111 (3D) & $5.000 / 5.000$ & 13.432 & -4.925 & 4.514 & 1.795 & $5 / 5$ \\
\hline 112 (3D) & $5.000 / 5.000$ & -21.037 & -4.780 & -7.567 & 1.763 & $4 / 4$ \\
\hline $9(3 \mathrm{D})$ & $5.000 / 5.000$ & -11.057 & 18.153 & 47.120 & 0.589 & $3 / 3$ \\
\hline $1(3 \mathrm{D})$ & $5.000 / 5.000$ & 19.985 & 3.609 & -7.538 & 2.108 & $5 / 5$ \\
\hline Mean $[\mathrm{m}]$ & & 1.976935 & -2.487704 & 7.450809 & & \\
\hline Sigma $[\mathrm{m}]$ & & 15.788648 & 13.855867 & 18.344893 & & \\
\hline RMS Error [m] & & 15.911935 & 14.077419 & 19.800243 & & \\
\hline
\end{tabular}

\begin{tabular}{|l|l|l|l|l|l|l|}
\hline $\begin{array}{l}\text { Check Point } \\
\text { Name }\end{array}$ & $\begin{array}{l}\text { Accuracy } \\
\text { XYIZ }[\mathrm{m}]\end{array}$ & $\begin{array}{l}\text { Error X } \\
{[\mathrm{m}]}\end{array}$ & $\begin{array}{l}\text { Error } \mathrm{Y} \\
{[\mathrm{m}]}\end{array}$ & $\begin{array}{l}\text { Error Z } \\
{[\mathrm{m}]}\end{array}$ & $\begin{array}{l}\text { Projection Error } \\
{[\text { pixel] }}\end{array}$ & Verified/Marked \\
\hline 205 & $5.0000 / 5.0000$ & -14.5457 & 20.7117 & 10.9595 & 1.7747 & $3 / 3$ \\
\hline 103 & $0.0200 / 0.0200$ & -28.9508 & -1.8392 & -1.2803 & 0.6101 & $2 / 2$ \\
\hline 211 & $0.0200 / 0.0200$ & $\mathrm{n} / \mathrm{a}$ & $\mathrm{n} / \mathrm{a}$ & $\mathrm{n} / \mathrm{a}$ & $\mathrm{n} / \mathrm{a}$ & $1 / 1$ \\
\hline 215 & $5.0000 / 5.0000$ & -29.7587 & 2.5650 & 6.8571 & 5.5642 & $2 / 2$ \\
\hline 216 & $5.0000 / 5.0000$ & 21.4606 & 0.3995 & 6.3561 & 2.3844 & $5 / 5$ \\
\hline 217 & $5.0000 / 5.0000$ & 9.0889 & -1.4667 & 6.8325 & 3.7135 & $5 / 5$ \\
\hline 229 & $0.0200 / 0.0200$ & 6.3228 & -3.0978 & 4.4006 & 2.4667 & $2 / 2$ \\
\hline 230 & $5.0000 / 5.0000$ & -11.3998 & -13.9504 & 3.9055 & 1.4003 & $5 / 5$ \\
\hline 219 & $5.0000 / 5.0000$ & 16.6176 & -31.0662 & 1.0065 & 3.5545 & $3 / 3$ \\
\hline 223 & $5.0000 / 5.0000$ & 22.9440 & -35.8526 & 73.7963 & 0.8631 & $3 / 3$ \\
\hline
\end{tabular}




\begin{tabular}{|c|c|c|c|c|c|c|c|c|}
\hline 221 & \multicolumn{2}{|c|}{$5.0000 / 5.0000$} & -6.7090 & -22.6182 & 31.5341 & \multicolumn{2}{|l|}{1.9595} & $4 / 4$ \\
\hline 204 & \multicolumn{2}{|c|}{$0.0200 / 0.0200$} & -8.4099 & 13.3683 & 5.2023 & \multicolumn{2}{|l|}{1.0421} & $2 / 2$ \\
\hline 207 & \multicolumn{2}{|c|}{$5.0000 / 5.0000$} & 16.3895 & 14.3538 & -11.3907 & \multicolumn{2}{|l|}{3.0286} & $3 / 3$ \\
\hline 201 & \multicolumn{2}{|c|}{$5.0000 / 5.0000$} & 9.5446 & 25.2405 & 1.2694 & \multicolumn{2}{|l|}{1.3953} & $3 / 3$ \\
\hline 225 & \multicolumn{2}{|c|}{$5.0000 / 5.0000$} & -28.3802 & 13.5068 & 4.1088 & \multicolumn{2}{|l|}{0.5093} & $2 / 2$ \\
\hline 222 & \multicolumn{2}{|c|}{$5.0000 / 5.0000$} & 36.3848 & -43.8976 & 69.9247 & \multicolumn{2}{|l|}{1.1323} & $3 / 3$ \\
\hline 224 & \multicolumn{2}{|c|}{$5.0000 / 5.0000$} & 1.5219 & -27.8428 & 82.2324 & \multicolumn{2}{|l|}{0.9610} & $3 / 3$ \\
\hline 125 & \multicolumn{2}{|c|}{$0.0200 / 0.0200$} & 24.1346 & -51.0951 & 99.2193 & 1.1013 & & $3 / 3$ \\
\hline 122 & $0.0200 / 0.020$ & & 32.0738 & -41.2438 & 57.0896 & 1.1428 & & $2 / 2$ \\
\hline 124 & $0.0200 / 0.020$ & & -3.5582 & -24.7870 & 55.8763 & 1.0068 & & $2 / 2$ \\
\hline 120 & $0.0200 / 0.020$ & & -13.0260 & -22.5107 & 26.6601 & 1.8390 & & $2 / 2$ \\
\hline 118 & $0.0200 / 0.020$ & & -1.9548 & -10.0429 & 0.1690 & 3.7513 & & $5 / 5$ \\
\hline 220 & $0.0200 / 0.020$ & & -33.7241 & -21.3843 & 25.3721 & 0.7099 & & $2 / 2$ \\
\hline 114 & $0.0200 / 0.020$ & & 26.7918 & -9.6736 & -9.0675 & 0.8393 & & $2 / 2$ \\
\hline 110 & $5.0000 / 5.000$ & & 43.9165 & -3.5220 & 22.2418 & 2.0539 & & $2 / 2$ \\
\hline 115 & $0.0200 / 0.020$ & & 9.9660 & -2.4166 & 10.0270 & 1.2210 & & $2 / 2$ \\
\hline 116 & $0.0200 / 0.020$ & & 0.3462 & -16.1175 & 0.6476 & 2.3614 & & $3 / 3$ \\
\hline 117 & $0.0200 / 0.020$ & & -25.3690 & -16.7141 & 5.1077 & 2.3516 & & $3 / 3$ \\
\hline 108 & $0.0200 / 0.020$ & & -23.5078 & -0.9310 & 0.7567 & 0.4017 & & $2 / 2$ \\
\hline 107 & $0.0200 / 0.020$ & & -0.9763 & -1.8617 & -3.1122 & 0.5252 & & $3 / 3$ \\
\hline 109 & $0.0200 / 0.020$ & & 12.0895 & -1.0513 & 2.4689 & 0.7458 & & $2 / 2$ \\
\hline 213 & $0.0200 / 0.020$ & & -1.1896 & 11.5416 & 8.0885 & 3.5217 & & $4 / 4$ \\
\hline 100 & $0.0200 / 0.020$ & & 17.9372 & 10.6993 & -15.3190 & 0.5098 & & $3 / 3$ \\
\hline 209 & $0.0200 / 0.020$ & & 0.9106 & 0.2640 & -4.0857 & 0.3468 & & $2 / 2$ \\
\hline 206 & $0.0200 / 0.020$ & & -23.4372 & 20.2612 & 13.1824 & 0.6105 & & $2 / 2$ \\
\hline 202 & $0.0200 / 0.020$ & & 24.0708 & 31.4907 & 4.5362 & 0.6520 & & $2 / 2$ \\
\hline 113 & $5.0000 / 5.000$ & & -49.0020 & 2.6763 & 6.4573 & 0.8380 & & $3 / 3$ \\
\hline 105 & $5.0000 / 5.000$ & & -25.5891 & 0.0996 & 0.0121 & 1.7713 & & $6 / 6$ \\
\hline 119 & $5.0000 / 5.000$ & & -13.8700 & -12.8703 & 7.8383 & 1.8031 & & $5 / 5$ \\
\hline 214 & $5.0000 / 5.000$ & & -29.9032 & 4.5561 & 1.1054 & 3.0766 & & $3 / 3$ \\
\hline 218 & $0.0200 / 0.020$ & & -19.7995 & -6.6707 & 1.9683 & 1.7652 & & $3 / 3$ \\
\hline 4 & $5.0000 / 5.000$ & & 18.2431 & -3.1278 & 7.1505 & 2.2120 & & $6 / 6$ \\
\hline 212 & $5.0000 / 5.000$ & & 18.0298 & 10.2731 & -5.3638 & 1.6519 & & $3 / 3$ \\
\hline Mean [m] & & & -0.564567 & -5.712661 & 14.296317 & & & \\
\hline Sigma [m] & & & 21.411469 & 18.402170 & 25.866447 & & & \\
\hline RMS Error [m] & & & 21.418911 & 19.268481 & 29.554319 & & & \\
\hline Localisation ac & $\begin{array}{r}\text { images } w \\
\text { imper and n }\end{array}$ & her & $\begin{array}{l}\text { rors in the th } \\
\text { e GCP has b }\end{array}$ & $\begin{array}{l}\text { e coordinate o } \\
\text { n automatical }\end{array}$ & $\begin{array}{l}\text { rections. The la } \\
\text { verified vs. me }\end{array}$ & $\begin{array}{l}\text { st column } \\
\text { nually mar }\end{array}$ & $\begin{array}{l}\text { ounts t } \\
\text { ed. }\end{array}$ & er of calibrated \\
\hline (?) Absolute Ge & olocation Varia & nce & & & & & & i \\
\hline Min Error [m] & Max Error [m] & $\mathrm{Ge}$ & location Error & $X[\%]$ & olocation Erro & $\mathrm{Y}[\%]$ & Geolo & n Error Z [\%] \\
\hline- & -150.00 & 3.5 & & & .71 & & 0.00 & \\
\hline-150.00 & -120.00 & 0.0 & & 3. & & & 0.00 & \\
\hline-120.00 & -90.00 & 0.0 & & 7. & & & 3.57 & \\
\hline-90.00 & -60.00 & 3.5 & & & .71 & & 3.57 & \\
\hline
\end{tabular}




\begin{tabular}{|c|c|c|c|c|}
\hline-60.00 & -30.00 & 17.86 & 3.57 & 14.29 \\
\hline-30.00 & 0.00 & 21.43 & 14.29 & 25.00 \\
\hline 0.00 & 30.00 & 32.14 & 0.00 & 28.57 \\
\hline 30.00 & 60.00 & 10.71 & 10.71 & 21.43 \\
\hline 60.00 & 90.00 & 7.14 & 17.86 & 3.57 \\
\hline 90.00 & 120.00 & 0.00 & 0.00 & 0.00 \\
\hline 120.00 & 150.00 & 0.00 & 10.71 & 0.00 \\
\hline 150.00 & - & 3.57 & 10.71 & 0.00 \\
\hline \multicolumn{2}{|c|}{ Mean [m] } & -24.820734 & -95.930104 & -638.096208 \\
\hline \multicolumn{2}{|c|}{ Sigma $[\mathrm{m}]$} & 68.699405 & 135.011329 & 42.590532 \\
\hline \multicolumn{2}{|c|}{$\begin{array}{l}\text { RMS Error } \\
\text { [m] }\end{array}$} & 73.045720 & 165.621991 & 639.516008 \\
\hline \multicolumn{5}{|c|}{$\begin{array}{l}\text { Min Error and Max Error represent geolocation error intervalsbetween }-1.5 \text { and } 1.5 \text { times the maximum accuracy of all the images. } \\
\text { Columns } X, Y, Z \text { show the percentage of images with geolocation errors within the predefined error intervals. The geolocation error is } \\
\text { the difference between the intial and computed image positions. Note that the image geolocation errors do not correspond to the } \\
\text { accuracy of the observed 3D points. }\end{array}$} \\
\hline \multicolumn{2}{|c|}{ Geolocation Bias } & $x$ & $\mathrm{Y}$ & Z \\
\hline \multicolumn{2}{|c|}{ Translation [m] } & -24.820734 & -95.930104 & -638.096215 \\
\hline
\end{tabular}

(?) Relative Geolocation Variance

\begin{tabular}{|l|l|l|l|}
\hline Relative Geolocation Error & Images X [\%] & Images Y [\%] & Images Z [\%] \\
\hline$[-1.00,1.00]$ & 75.00 & 21.43 & 96.43 \\
\hline$[-2.00,2.00]$ & 92.86 & 57.14 & 100.00 \\
\hline$[-3.00,3.00]$ & 92.86 & 78.57 & 100.00 \\
\hline Mean of Geolocation Accuracy [m] & 50.000000 & 50.000000 & 100.000000 \\
\hline Sigma of Geolocation Accuracy [m] & 0.000000 & 0.000000 & 0.000000 \\
\hline
\end{tabular}

Images $\mathrm{X}, \mathrm{Y}, \mathrm{Z}$ represent the percentage of images with a relative geolocation error in $\mathrm{X}, \mathrm{Y}, \mathrm{Z}$.

Initial Processing Details

System Information

\begin{tabular}{|l|l|}
\hline \multirow{3}{*}{ Hardware } & CPU: Intel(R) Core(TM) i7-6820HQ CPU @ 2.70GHz \\
& RAM: $16 \mathrm{~GB}$ \\
& GPU: Intel(R) HD Graphics 530 (Driver: 20.19.15.4331) \\
\hline Operating System & Windows 10 Enterprise, 64-bit \\
\hline
\end{tabular}

Coordinate Systems

\begin{tabular}{|l|l|}
\hline Image Coordinate System & NAD83(2011) / UTM zone 11N (egm96) \\
\hline Ground Control Point (GCP) Coordinate System & NAD83(2011) / UTM zone 11N (2D) \\
\hline Output Coordinate System & NAD83(2011) / UTM zone 11N (2D) \\
\hline
\end{tabular}

Processing Options

Detected Template

Keypoints Image Scale

Advanced: Matching Image Pairs

Advanced: Matching Strategy

Advanced: Keypoint Extraction

Advanced: Calibration
No Template Available

Full, Image Scale: 0.5

Aerial Grid or Corridor

Use Geometrically Verified Matching: no

Targeted Number of Keypoints: Automatic

Calibration Method: Standard

Internal Parameters Optimization: All

External Parameters Optimization: All

Rematch: Auto, yes 
Pix4D Professional Quality Report for Minimum Control processing session Quality Report

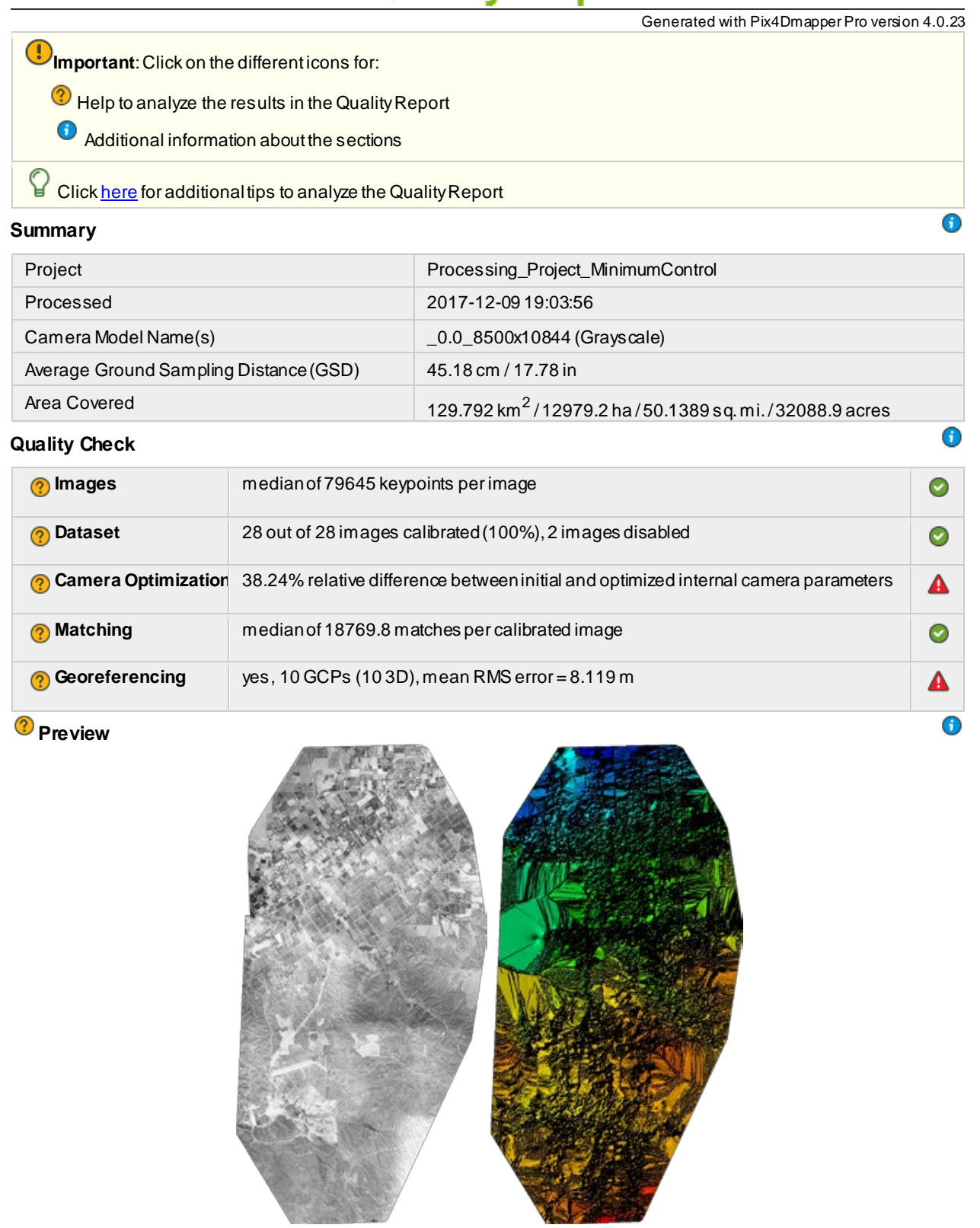

Figure 1: Orthomosaic and the corresponding sparse Digital Surface Model (DSM) before densification.

\section{Calibration Details}


(?) Initial Image Positions

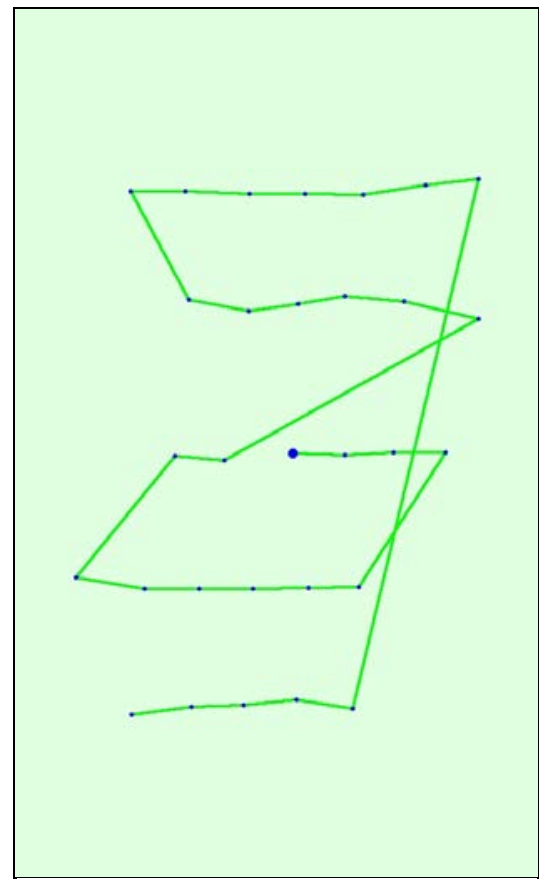

Figure 2: Top view of the initial image position. The green line follows the position of the images in time starting from the large blue dot.

(3) Computed Image/GCPs/Manual Tie Points Positions

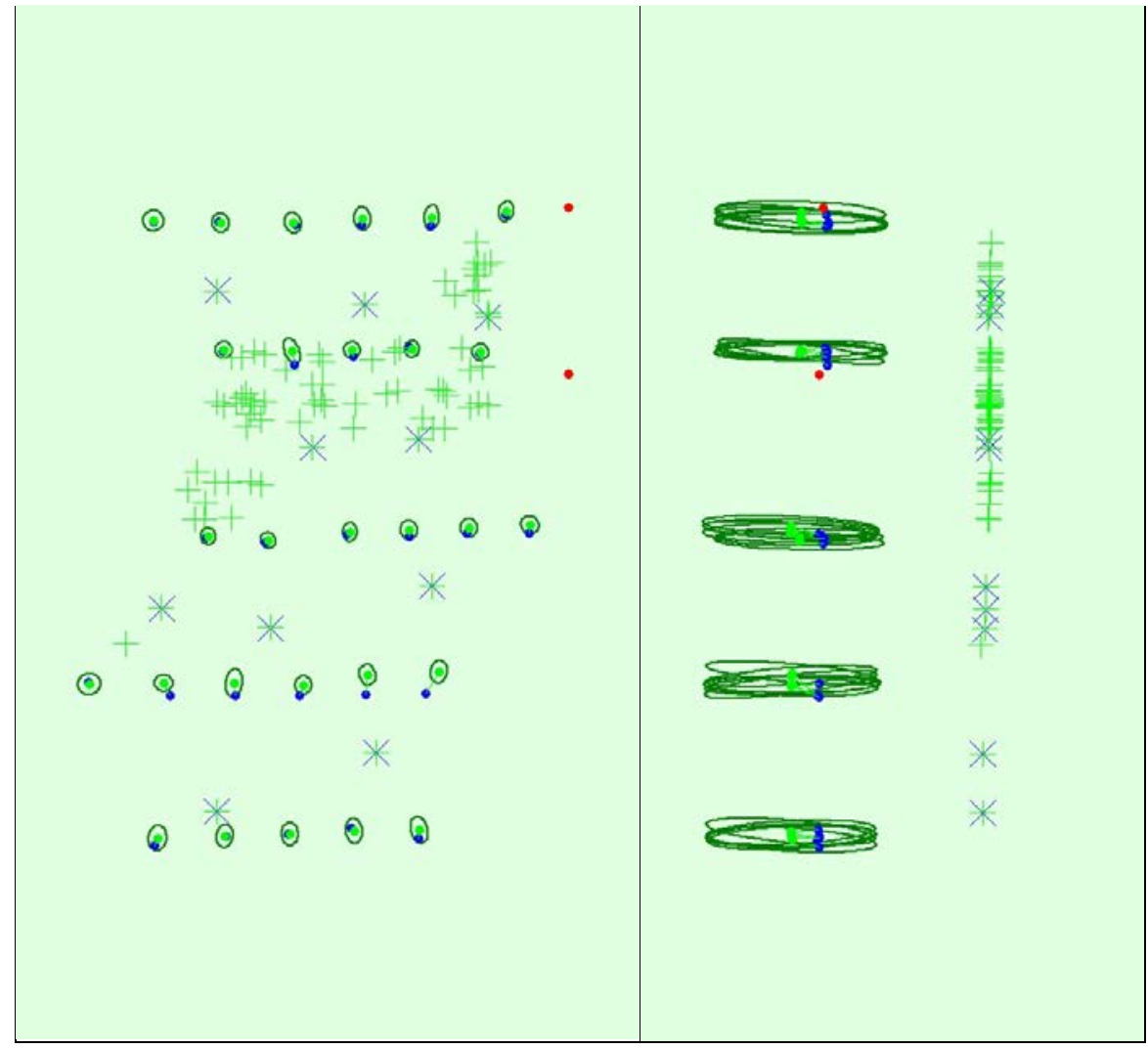




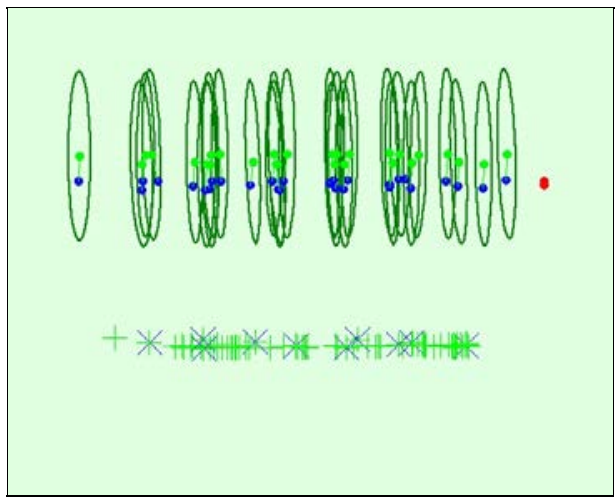

Uncertainty ellipses 100x magnified

Figure 3: Offset between initial (blue dots) and computed (green dots) image positions as well as the offset between the GCPs initial positions (blue crosses) and heir computed positions (green crosses) in the top-View (XY plane), front-view (XZ plane), and side-view (YZ plane). Red dots indicate disabled or uncalibrated images. Dark green ellipses indicate the absolute position uncertainty of the

(?) Absolute camera position and orientation uncertainties

\begin{tabular}{|l|l|l|l|l|l|l|}
\hline & $X[\mathrm{~m}]$ & $Y[\mathrm{~m}]$ & $Z[\mathrm{~m}]$ & Omega [degree] & Phi [degree] & Kappa [degree] \\
\hline Mean & 1.931 & 2.281 & 18.800 & 0.016 & 0.015 & 0.005 \\
\hline Sigma & 0.198 & 0.347 & 0.240 & 0.005 & 0.004 & 0.002 \\
\hline
\end{tabular}

(3) Overlap

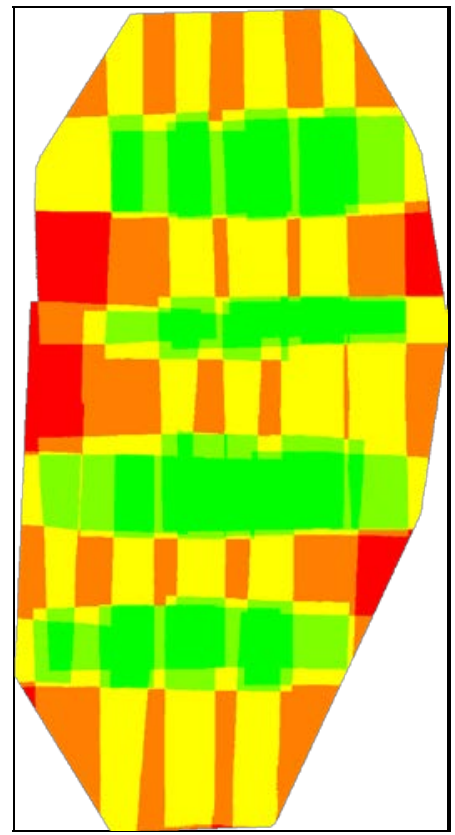

Number of overlapping images: $2 \quad 3 \quad 4 \quad 5+$

Figure 4: Number of ov erlapping images computed for each pixel of the orthomosaic.

Red and yellow areas indicate low overlap for which poor results may be generated. Green areas indicate an ov erlap of over 5 images (see Figure 5 for keypoint matches).

\section{Bundle Block Adjustment Details}


(?) Internal Camera Parameters_0.0_8500x10844 (Grayscale). Sensor Dimensions: 25.400 [mm] x 32.404 $[\mathrm{mm}]$

[6] XIF ID: $0.0 \_8500 \times 10844$

\begin{tabular}{|c|c|c|c|c|c|c|c|c|}
\hline & $\begin{array}{l}\text { Focal } \\
\text { Length }\end{array}$ & $\begin{array}{l}\text { Principal } \\
\text { Point } x\end{array}$ & $\begin{array}{l}\text { Principal } \\
\text { Pointy }\end{array}$ & R1 & $\mathrm{R} 2$ & R3 & $\mathrm{T} 1$ & $\mathrm{~T} 2$ \\
\hline Initial Values & $\begin{array}{l}6692.914 \\
\text { [pixel] } \\
20.000[\mathrm{~mm}]\end{array}$ & $\begin{array}{l}4250.000 \\
\text { [pixel] } \\
12.700[\mathrm{~mm}]\end{array}$ & $\begin{array}{l}5422.001 \\
\text { [pixel] } \\
16.202[\mathrm{~mm}]\end{array}$ & 0.000 & 0.000 & 0.000 & 0.000 & 0.000 \\
\hline Optimized Values & $\begin{array}{l}9252.677 \\
\text { [pixel] } \\
27.649[\mathrm{~mm}]\end{array}$ & $\begin{array}{l}4373.535 \\
\text { [pixel] } \\
13.069[\mathrm{~mm}]\end{array}$ & $\begin{array}{l}5389.396 \\
\text { [pixel] } \\
16.105[\mathrm{~mm}]\end{array}$ & $\overline{-} .004$ & $\overline{-} .003$ & 0.015 & $\overline{-} .000$ & 0.001 \\
\hline $\begin{array}{l}\text { Uncertainties } \\
\text { (Sigma) }\end{array}$ & $\begin{array}{l}41.646 \text { [pixel] } \\
0.124[\mathrm{~mm}]\end{array}$ & $\begin{array}{l}3.722[\text { pixel] } \\
0.011[\mathrm{~mm}]\end{array}$ & $\begin{array}{l}4.100[\text { pixel] } \\
0.012[\mathrm{~mm}]\end{array}$ & 0.000 & 0.002 & 0.002 & 0.000 & 0.000 \\
\hline
\end{tabular}

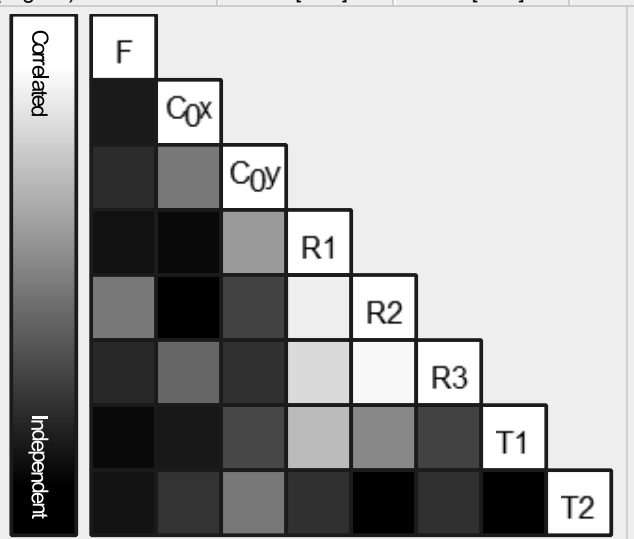

The correlation between camerainternal parameters determined by the bundle adjustment. White indicates a full correlation between the parameters, ie. any change in one can be fullycompensated by the other. Black indicates that the parameter is completelyindependent, and is not affected by other parameters.

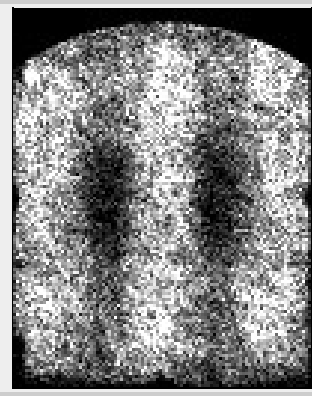

The number of Automatic Tie Points (ATPs) per pixel, averaged over all images of the cameramodel, is color coded between black and white. White indicates that, on average, more than 16 ATPs have been extracted at the pixel location. Blackindicates that, on average, 0 ATPs have been extracted at the pixel location. Click on the image to the see the average direction and magnitude of the re-

projection error for each pixel. Note that the vectors are scaledfor better visualization. The scale bar indicates the magnitude of 1 pixel error.

(3) $2 \mathrm{D}$ Keypoints Table

\begin{tabular}{|l|l|l|}
\hline & Number of 2D Keypoints per Image & Number of Matched 2D Keypoints per Image \\
\hline Median & 79645 & 18770 \\
\hline Min & 66668 & 8858 \\
\hline Max & 86825 & 27661 \\
\hline Mean & 79224 & 18642 \\
\hline ? $3 D$ Points from 2D Keypoint Matches & \\
\hline
\end{tabular}

\begin{tabular}{|l|l|}
\hline & Number of 3D Points Observed \\
\hline In 2 Images & 208723 \\
\hline In 3 Images & 28375 \\
\hline In 4 Images & 3342 \\
\hline In 5 Images & 990 \\
\hline In 6 Images & 183 \\
\hline
\end{tabular}




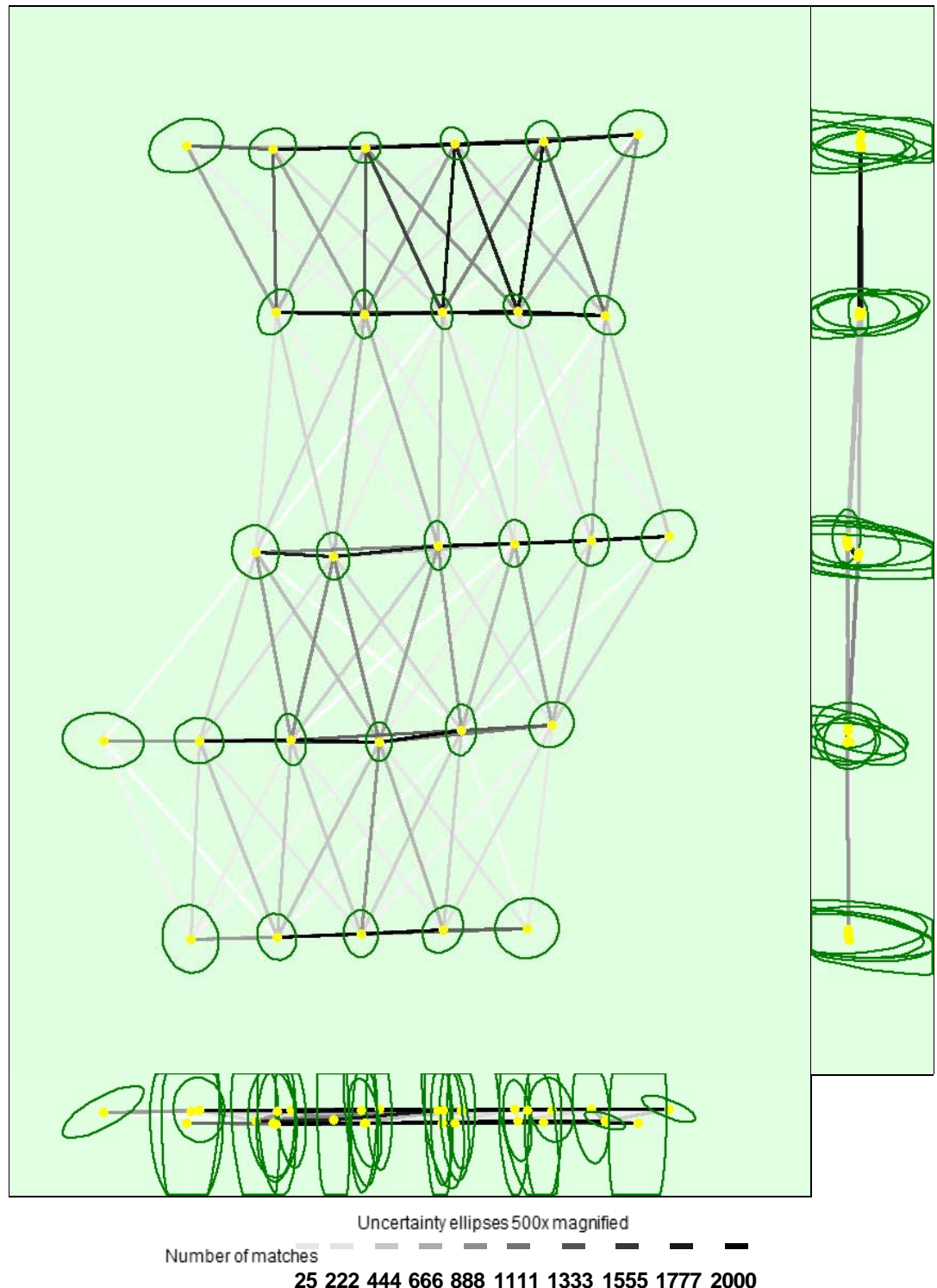

Figure 5: Computed image positions with links between matched images. The darkness of the links indicates the number of matched $2 D$ keypoints between the images. Bright links indicate weak links and require manual tie points or more images. Dark green ellipses indicate the relative camera position uncertainty of the bundle block adjustment result.

(?) Relative camera position and orientation uncertainties

\begin{tabular}{|l|l|l|l|l|l|l|}
\hline & $X[\mathrm{~m}]$ & $Y[\mathrm{~m}]$ & $Z[\mathrm{~m}]$ & Omega [degree] & Phi [degree] & Kappa [degree] \\
\hline Mean & 0.702 & 0.800 & 2.367 & 0.065 & 0.039 & 0.008 \\
\hline Sigma & 0.251 & 0.135 & 1.105 & 0.037 & 0.022 & 0.003 \\
\hline
\end{tabular}

(?) Manual Tie Points

\begin{tabular}{|l|l|l|}
\hline MTP Name & Projection Error [pixel] & Verified/Marked \\
\hline 509 & 1.523 & $2 / 2$ \\
\hline 510 & 0.607 & $2 / 2$ \\
\hline 511 & 1.630 & $3 / 3$ \\
\hline 512 & 0.546 & $2 / 2$ \\
\hline 513 & 2.191 & $3 / 3$ \\
\hline 514 & 1.264 & $2 / 2$ \\
\hline
\end{tabular}




\begin{tabular}{|c|c|c|}
\hline mtp90 & 1.386 & $2 / 2$ \\
\hline $\mathrm{mtp} 91$ & 1.215 & $2 / 2$ \\
\hline mtp92 & 1.882 & $3 / 3$ \\
\hline $\mathrm{mtp} 93$ & 1.188 & $2 / 2$ \\
\hline mtp94 & 1.242 & $2 / 2$ \\
\hline $\mathrm{mtp} 95$ & 1.813 & $2 / 2$ \\
\hline mtp96 & 1.467 & $2 / 2$ \\
\hline 515 & 1.317 & $2 / 2$ \\
\hline 516 & 1.088 & $2 / 2$ \\
\hline 517 & 1.439 & $3 / 3$ \\
\hline 518 & 1.473 & $2 / 2$ \\
\hline 519 & 1.347 & $2 / 2$ \\
\hline 520 & 0.006 & $2 / 2$ \\
\hline 521 & 1.061 & $2 / 2$ \\
\hline 522 & 0.386 & $2 / 2$ \\
\hline 523 & 0.625 & $2 / 2$ \\
\hline 524 & 0.974 & $2 / 2$ \\
\hline 525 & 0.262 & $2 / 2$ \\
\hline 526 & 1.858 & $2 / 2$ \\
\hline 527 & 1.253 & $2 / 2$ \\
\hline 528 & 1.023 & $2 / 2$ \\
\hline 529 & 0.970 & $2 / 2$ \\
\hline 530 & 1.454 & $2 / 2$ \\
\hline 531 & 2.076 & $2 / 2$ \\
\hline 532 & 1.404 & $3 / 3$ \\
\hline 533 & 1.921 & $2 / 2$ \\
\hline 534 & 0.832 & $2 / 2$ \\
\hline 535 & 0.811 & $2 / 2$ \\
\hline 536 & 0.485 & $2 / 2$ \\
\hline 537 & 0.614 & $2 / 2$ \\
\hline 538 & 0.568 & $2 / 2$ \\
\hline 539 & 0.734 & $2 / 2$ \\
\hline 540 & 1.212 & $2 / 2$ \\
\hline 541 & 0.983 & $2 / 2$ \\
\hline 542 & 0.456 & $2 / 2$ \\
\hline 543 & 0.209 & $2 / 2$ \\
\hline 544 & 0.933 & $3 / 3$ \\
\hline 545 & 0.108 & $2 / 2$ \\
\hline 546 & 1.050 & $2 / 2$ \\
\hline 549 & 2.899 & $5 / 5$ \\
\hline 550 & 1.587 & $3 / 3$ \\
\hline 551 & 1.322 & $2 / 2$ \\
\hline 552 & 0.026 & $2 / 2$ \\
\hline 553 & 0.939 & $3 / 3$ \\
\hline 554 & 2.066 & $5 / 5$ \\
\hline 556 & 1.786 & $6 / 6$ \\
\hline 559 & 0.130 & $2 / 2$ \\
\hline 562 & 0.267 & $3 / 3$ \\
\hline 564 & 2.319 & $4 / 4$ \\
\hline 565 & 1.933 & $4 / 4$ \\
\hline 566 & 1.901 & $4 / 4$ \\
\hline 567 & 1.396 & $4 / 4$ \\
\hline 568 & 0.954 & $4 / 4$ \\
\hline 569 & 2.273 & $4 / 4$ \\
\hline 570 & 1.377 & $4 / 4$ \\
\hline 571 & 2.058 & $4 / 4$ \\
\hline 572 & 2.315 & $5 / 5$ \\
\hline 573 & 2.328 & $3 / 3$ \\
\hline
\end{tabular}



574
2.267
$4 / 4$
Projection errors for manual tie points. The last column counts the number of images where the manual tie point has been

automaticallyv erified vs. manually marked.

Geolocation Details

(3) Ground Control Points

\begin{tabular}{|l|l|l|l|l|l|l|}
\hline GCP Name & $\begin{array}{l}\text { Accuracy XY/Z } \\
{[\mathrm{m}]}\end{array}$ & Error $X[\mathrm{~m}]$ & Error $Y[\mathrm{~m}]$ & Error Z [m] & $\begin{array}{l}\text { Projection Error } \\
{[\text { pixel] }}\end{array}$ & Verified/Marked \\
\hline $8(3 \mathrm{D})$ & $5.000 / 5.000$ & -15.600 & -4.076 & -0.051 & 1.871 & $5 / 5$ \\
\hline $6(3 \mathrm{D})$ & $5.000 / 5.000$ & 17.969 & -18.495 & 0.349 & 1.573 & $4 / 4$ \\
\hline $10(3 \mathrm{D})$ & $5.000 / 5.000$ & -3.155 & -16.023 & 0.851 & 2.187 & $5 / 5$ \\
\hline $2(3 \mathrm{D})$ & $5.000 / 5.000$ & 2.711 & 10.406 & 2.050 & 2.158 & $6 / 6$ \\
\hline $5(3 \mathrm{D})$ & $5.000 / 5.000$ & -13.595 & -0.310 & 2.100 & 2.187 & $5 / 5$ \\
\hline 7 (3D) & $5.000 / 5.000$ & 8.136 & -2.887 & -2.679 & 2.082 & $5 / 5$ \\
\hline 3 (3D) & $5.000 / 5.000$ & -17.704 & 5.808 & -2.539 & 2.197 & $4 / 4$ \\
\hline $111(3 \mathrm{D})$ & $5.000 / 5.000$ & 10.281 & -1.619 & 0.710 & 1.781 & $5 / 5$ \\
\hline 9 (3D) & $5.000 / 5.000$ & -3.047 & 8.149 & 0.772 & 0.586 & $3 / 3$ \\
\hline 1 (3D) & $5.000 / 5.000$ & 15.154 & 16.975 & 0.297 & 1.786 & $5 / 5$ \\
\hline Mean [m] & & 0.114999 & -0.207201 & 0.185935 & & \\
\hline Sigma [m] & & 12.216523 & 10.595281 & 1.546827 & & \\
\hline RMS Error [m] & & 12.217064 & 10.597307 & 1.557962 & & \\
\hline
\end{tabular}

\begin{tabular}{|c|c|c|c|c|c|c|}
\hline $\begin{array}{l}\text { Check Point } \\
\text { Name }\end{array}$ & $\begin{array}{l}\text { Accuracy } \\
\text { XY/Z [m] }\end{array}$ & $\begin{array}{l}\text { Error X } \\
{[\mathrm{m}]}\end{array}$ & $\begin{array}{l}\text { Error } Y \\
{[\mathrm{~m}]}\end{array}$ & $\begin{array}{l}\text { Error Z } \\
{[\mathrm{m}]}\end{array}$ & $\begin{array}{l}\text { Projection Error } \\
\text { [pixel] }\end{array}$ & Verified/Marked \\
\hline 205 & $5.0000 / 5.0000$ & 3.2658 & 32.2139 & 51.2122 & 1.4344 & $3 / 3$ \\
\hline 208 & $5.0000 / 5.0000$ & 8.0467 & 22.8343 & 16.0406 & 1.2899 & $3 / 3$ \\
\hline 103 & $0.0200 / 0.0200$ & -17.7071 & -1.1066 & 14.9852 & 0.5957 & $2 / 2$ \\
\hline 211 & $0.0200 / 0.0200$ & $\mathrm{n} / \mathrm{a}$ & $\mathrm{n} / \mathrm{a}$ & $\mathrm{n} / \mathrm{a}$ & $\mathrm{n} / \mathrm{a}$ & $1 / 1$ \\
\hline 215 & $5.0000 / 5.0000$ & -22.0139 & 0.1167 & 13.7624 & 5.7192 & $2 / 2$ \\
\hline 104 & $5.0000 / 5.0000$ & 7.2014 & 16.8111 & 1.5147 & 1.4416 & $6 / 6$ \\
\hline 226 & $0.0200 / 0.0200$ & 3.5112 & 3.1501 & -0.0704 & 2.2044 & $3 / 3$ \\
\hline 227 & $0.0200 / 0.0200$ & 8.6546 & 0.7888 & -0.6232 & 1.5581 & $3 / 3$ \\
\hline 216 & $5.0000 / 5.0000$ & 16.7356 & 3.9088 & 3.1641 & 2.1730 & $5 / 5$ \\
\hline 217 & $5.0000 / 5.0000$ & 6.4210 & 1.8774 & 3.4829 & 3.4973 & $5 / 5$ \\
\hline 228 & $0.0200 / 0.0200$ & -12.2778 & -5.0448 & -7.3249 & 1.3549 & $3 / 3$ \\
\hline 229 & $0.0200 / 0.0200$ & 3.0182 & -1.5301 & -0.3126 & 2.4797 & $2 / 2$ \\
\hline 230 & $5.0000 / 5.0000$ & -13.7704 & -12.2949 & -5.1382 & 1.6903 & $5 / 5$ \\
\hline 219 & $5.0000 / 5.0000$ & 15.0570 & -35.9954 & -12.1280 & 3.5209 & $3 / 3$ \\
\hline 223 & $5.0000 / 5.0000$ & 36.0854 & -41.4958 & 13.3874 & 0.8464 & $3 / 3$ \\
\hline 221 & $5.0000 / 5.0000$ & -4.5549 & -21.4307 & 0.1486 & 1.9888 & $4 / 4$ \\
\hline 204 & $0.0200 / 0.0200$ & 4.4850 & 25.5909 & 38.9464 & 1.0387 & $2 / 2$ \\
\hline 207 & $5.0000 / 5.0000$ & 15.0786 & 34.6129 & 17.2538 & 3.0274 & $3 / 3$ \\
\hline 201 & $5.0000 / 5.0000$ & 17.0951 & 46.4606 & 42.6300 & 1.4952 & $3 / 3$ \\
\hline 203 & $5.0000 / 5.0000$ & 8.6764 & 34.3879 & 42.6984 & 1.5892 & $3 / 3$ \\
\hline 225 & $5.0000 / 5.0000$ & -12.8061 & 16.8188 & 32.0891 & 0.4308 & $2 / 2$ \\
\hline
\end{tabular}




\begin{tabular}{|c|c|c|c|c|c|c|}
\hline 121 & $5.0000 / 5.0000$ & 40.4060 & -52.2586 & 11.5899 & 1.8465 & $3 / 3$ \\
\hline 222 & $5.0000 / 5.0000$ & 53.1720 & -55.7405 & 4.7983 & 1.1346 & $3 / 3$ \\
\hline 224 & $5.0000 / 5.0000$ & 16.1056 & -25.4256 & 12.5709 & 0.9792 & $3 / 3$ \\
\hline 125 & $0.0200 / 0.0200$ & 42.0524 & -54.0005 & 27.2215 & 1.1250 & $3 / 3$ \\
\hline 122 & $0.0200 / 0.0200$ & 41.0235 & -53.7844 & 8.5038 & 1.1953 & $2 / 2$ \\
\hline 123 & $0.0200 / 0.0200$ & 20.1848 & -35.6434 & 5.6774 & 0.1354 & $3 / 3$ \\
\hline 124 & $0.0200 / 0.0200$ & 3.3280 & -21.5592 & 3.6580 & 0.9492 & $2 / 2$ \\
\hline 120 & $0.0200 / 0.0200$ & -12.7493 & -19.2181 & -1.3938 & 1.8141 & $2 / 2$ \\
\hline 118 & $0.0200 / 0.0200$ & -2.6967 & -12.0773 & -10.6342 & 2.9368 & $5 / 5$ \\
\hline 220 & $0.0200 / 0.0200$ & -36.8691 & -32.5120 & 9.5886 & 0.5917 & $2 / 2$ \\
\hline 114 & $0.0200 / 0.0200$ & 19.5774 & -10.6566 & -9.8674 & 0.9036 & $2 / 2$ \\
\hline 110 & $5.0000 / 5.0000$ & 34.2272 & 0.1707 & 21.5217 & 1.6979 & $2 / 2$ \\
\hline 115 & $0.0200 / 0.0200$ & 6.5535 & -2.0871 & 4.7012 & 1.2079 & $2 / 2$ \\
\hline 116 & $0.0200 / 0.0200$ & -2.0508 & -15.0016 & -5.4171 & 2.3606 & $3 / 3$ \\
\hline 117 & $0.0200 / 0.0200$ & -28.7354 & -16.6084 & -0.3558 & 2.2710 & $3 / 3$ \\
\hline 108 & $0.0200 / 0.0200$ & -21.4808 & 0.3117 & 1.4952 & 0.4269 & $2 / 2$ \\
\hline 107 & $0.0200 / 0.0200$ & -1.4087 & 3.7962 & -1.9810 & 0.5081 & $3 / 3$ \\
\hline 109 & $0.0200 / 0.0200$ & 7.7568 & 4.9858 & 1.8288 & 0.7037 & $2 / 2$ \\
\hline 210 & $0.0200 / 0.0200$ & -8.1769 & 13.8715 & 16.9687 & 0.7622 & $3 / 3$ \\
\hline 213 & $0.0200 / 0.0200$ & 5.1374 & 18.6351 & 18.1065 & 2.9735 & $4 / 4$ \\
\hline 100 & $0.0200 / 0.0200$ & 15.5435 & 27.7883 & 4.7033 & 0.5092 & $3 / 3$ \\
\hline 209 & $0.0200 / 0.0200$ & 5.6340 & 12.4538 & 14.7966 & 0.3502 & $2 / 2$ \\
\hline 101 & $0.0200 / 0.0200$ & 10.5724 & 27.4129 & 30.5684 & 2.1924 & $3 / 3$ \\
\hline 206 & $0.0200 / 0.0200$ & -4.3236 & 26.9933 & 51.0006 & 0.7011 & $2 / 2$ \\
\hline 202 & $0.0200 / 0.0200$ & 35.0948 & 49.5772 & 45.2886 & 0.8056 & $2 / 2$ \\
\hline 113 & $5.0000 / 5.0000$ & -50.9866 & -0.8087 & 4.2919 & 0.6601 & $3 / 3$ \\
\hline 105 & $5.0000 / 5.0000$ & -19.4473 & 4.5311 & 7.5437 & 1.6857 & $6 / 6$ \\
\hline 119 & $5.0000 / 5.0000$ & -15.7120 & -12.1405 & -1.2644 & 1.3815 & $5 / 5$ \\
\hline 214 & $5.0000 / 5.0000$ & -21.2750 & 3.6543 & 9.4607 & 3.0100 & $3 / 3$ \\
\hline 218 & $0.0200 / 0.0200$ & -22.2125 & -6.6952 & -2.9120 & 1.7665 & $3 / 3$ \\
\hline 4 & $5.0000 / 5.0000$ & 14.2377 & 0.7178 & 4.0793 & 2.0872 & $6 / 6$ \\
\hline 112 & $5.0000 / 5.0000$ & -22.8831 & -5.0905 & -12.1095 & 1.8827 & $4 / 4$ \\
\hline 212 & $5.0000 / 5.0000$ & 11.3778 & 23.5497 & 0.9979 & 1.6540 & $3 / 3$ \\
\hline $\operatorname{Mean}[\mathrm{m}]$ & & 3.355163 & -1.707125 & 10.013797 & & \\
\hline Sigma [m] & & 20.740417 & 24.839994 & 15.858482 & & \\
\hline RMS Error $[\mathrm{m}]$ & & 21.010046 & 24.898586 & 18.755468 & & \\
\hline
\end{tabular}

(3) Absolute Geolocation Variance

\begin{tabular}{|l|l|l|l|l|}
\hline Min Error $[\mathrm{m}]$ & Max Error $[\mathrm{m}]$ & GeolocationError X [\%] & Geolocation Error Y [\%] & Geolocation Error Z [\%] \\
\hline- & -150.00 & 3.57 & 14.29 & 0.00 \\
\hline-150.00 & -120.00 & 0.00 & 7.14 & 0.00 \\
\hline-120.00 & -90.00 & 0.00 & 0.00 & 3.57 \\
\hline-90.00 & -60.00 & 3.57 & 10.71 & 3.57 \\
\hline
\end{tabular}




\begin{tabular}{|c|c|c|c|c|}
\hline-60.00 & -30.00 & 17.86 & 3.57 & 10.71 \\
\hline-30.00 & 0.00 & 21.43 & 7.14 & 28.57 \\
\hline 0.00 & 30.00 & 32.14 & 7.14 & 32.14 \\
\hline 30.00 & 60.00 & 7.14 & 10.71 & 21.43 \\
\hline 60.00 & 90.00 & 10.71 & 3.57 & 0.00 \\
\hline 90.00 & 120.00 & 0.00 & 17.86 & 0.00 \\
\hline 120.00 & 150.00 & 0.00 & 3.57 & 0.00 \\
\hline 150.00 & - & 3.57 & 14.29 & 0.00 \\
\hline \multicolumn{2}{|c|}{ Mean [m] } & -26.980554 & -120.085395 & -582.064328 \\
\hline \multicolumn{2}{|c|}{ Sigma [m] } & 67.868828 & 135.792320 & 36.205191 \\
\hline \multicolumn{2}{|c|}{$\begin{array}{l}\text { RMS Error } \\
{[\mathrm{m}]}\end{array}$} & 73.035115 & 181.273430 & 583.189247 \\
\hline \multicolumn{5}{|c|}{$\begin{array}{l}\text { Min Error and Max Error represent geolocation error interv alsbetween }-1.5 \text { and } 1.5 \text { times the maximum accuracy of all the images. } \\
\text { Columns } X, Y, Z \text { show the percentage of images with geolocation errors within the predefined error intervals. The geolocation error is } \\
\text { the difference between the intial and computed image positions. Note that the image geolocation errors do not correspond to the } \\
\text { accuracy of the observed 3D points. }\end{array}$} \\
\hline \multicolumn{2}{|c|}{ Geolocation Bias } & $x$ & $\mathrm{Y}$ & z \\
\hline \multicolumn{2}{|c|}{ Translation [m] } & -26.980554 & -120.085395 & -582.064328 \\
\hline
\end{tabular}

(?) Relative Geolocation Variance

\begin{tabular}{|l|l|l|l|}
\hline Relative Geolocation Error & Images X[\%] & Images Y [\%] & Images Z[\%] \\
\hline$[-1.00,1.00]$ & 78.57 & 17.86 & 96.43 \\
\hline$[-2.00,2.00]$ & 92.86 & 53.57 & 100.00 \\
\hline$[-3.00,3.00]$ & 92.86 & 71.43 & 100.00 \\
\hline Mean of Geolocation Accuracy[m] & 50.000000 & 50.000000 & 100.000000 \\
\hline Sigma of Geolocation Accuracy[m] & 0.000000 & 0.000000 & 0.000000 \\
\hline
\end{tabular}

Images $\mathrm{X}, \mathrm{Y}, \mathrm{Z}$ represent the percentage of images with a relativ e geolocation error in $\mathrm{X}, \mathrm{Y}, \mathrm{Z}$.

\section{Initial Processing Details}

System Information

\begin{tabular}{|l|l|}
\hline Hardware & $\begin{array}{l}\text { CPU: Intel(R) Core(TM) i7-6820HQ CPU @ 2.70GHz } \\
\text { RAM: 16GB } \\
\text { GPU: Intel(R) HD Graphics 530 (Driver: 20.19.15.4331) }\end{array}$ \\
\hline Operating System & Windows 10 Enterprise,64-bit \\
\hline Coordinate Systems &
\end{tabular}

Image Coordinate System

Ground Control Point (GCP) Coordinate System

NAD83(2011)/UTM zone 11N (egm96)

Output Coordinate System

NAD83(2011)/UTM zone 11N (2D)

NAD83(2011)/UTM zone 11N (2D)

Processing Options

Detected Template

Keypoints Image Scale

Advanced: Matching Image Pairs

Advanced: Matching Strategy

Advanced: Keypoint Extraction

Advanced: Calibration

No Template Available

Full, Image Scale: 0.5

Aerial Grid or Corridor

Use Geometrically Verified Matching: no

Targeted Number of Keypoints: Automatic

Calibration Method: Standard

Internal Parameters Optimization: All

External Parameters Optimization: All

Rematch: Auto, yes 


\section{Appendix C. Python Image Standardization Script}

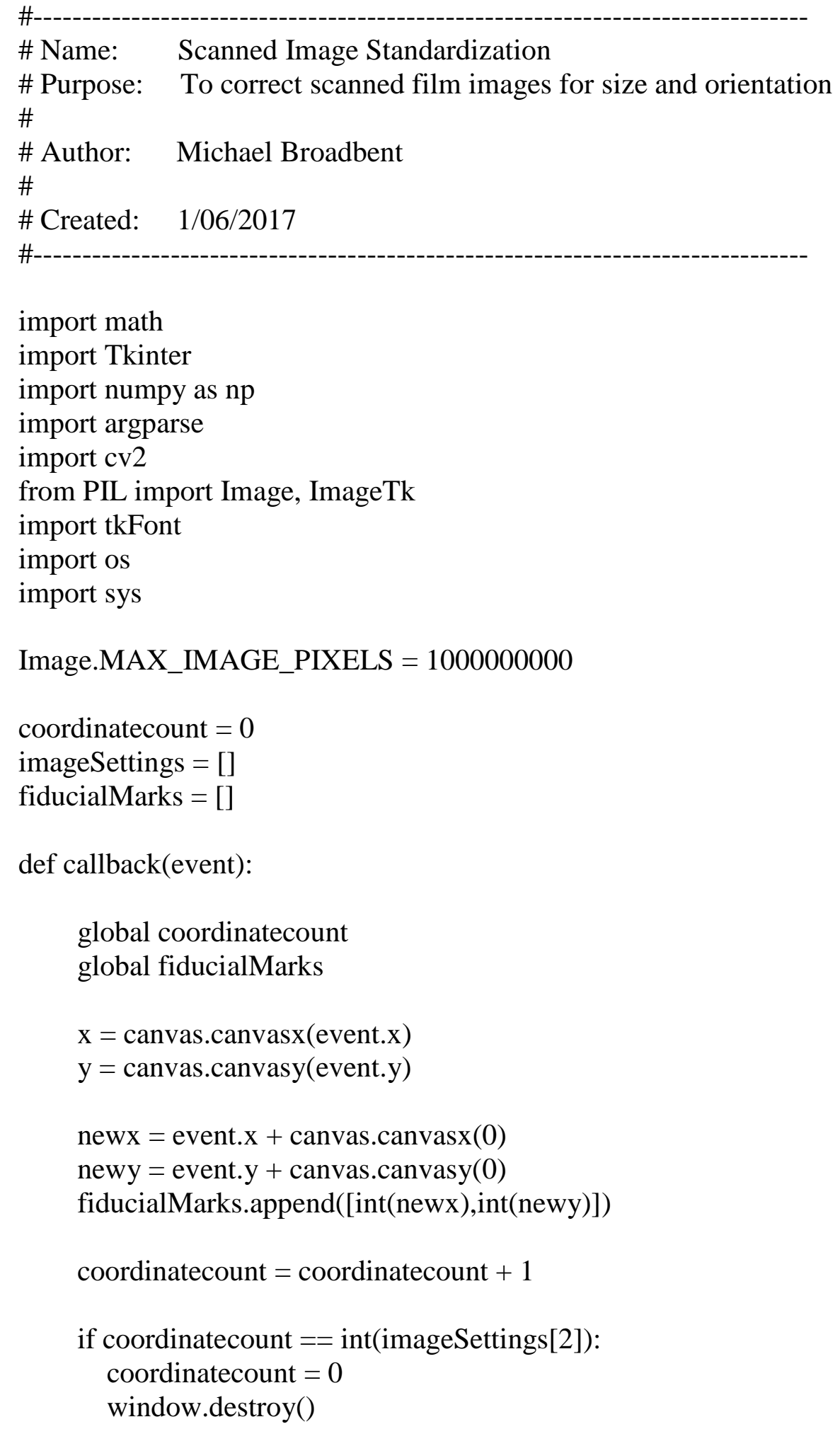


\#Function to load the config file and set the global script variables def loadconfig():

global imageSettings

fin=open(sys.path[0] + "'config.ini",'r')

for line in fin:

inputline = line.split(",")

imageSettings.append(inputline[1].strip('In'))

loadconfig()

directory $=$ imageSettings $[6]$

\#Make an output directory if it doesn't already exist

try:

os.stat(directory + "\Output")

except:

os.mkdir(directory + "\Output")

for filename in os.listdir(directory):

\#\#***Tkinter section enclosed in $* * *$ inspired by

https://stackoverflow.com/questions/33974204/python-tkinter-moving-images-onbuttonpress

if filename.endswith(imageSettings[0]):

globalfilepath $=$ os.path.join(directory, filename)

window $=$ Tkinter. $\operatorname{Tk}()$

window.geometry("1200x600")

window.update()

canvas $=$ Tkinter.Canvas $($ window $)$

top $=$ Tkinter.Frame $($ window $)$

top.pack(side="top")

bottom $=$ Tkinter.Frame(window)

bottom.pack(side="bottom")

image = Image.open(globalfilepath)

canvas.pack(fill=Tkinter.BOTH, expand=2)

xscrollbar = Tkinter.Scrollbar(window, orient="horizontal", command $=$ canvas. xview)

yscrollbar = Tkinter.Scrollbar(window, orient="vertical", command=canvas.yview)

xscrollbar.pack(side="bottom", fill="x")

yscrollbar.pack(side="right", fill="y") 


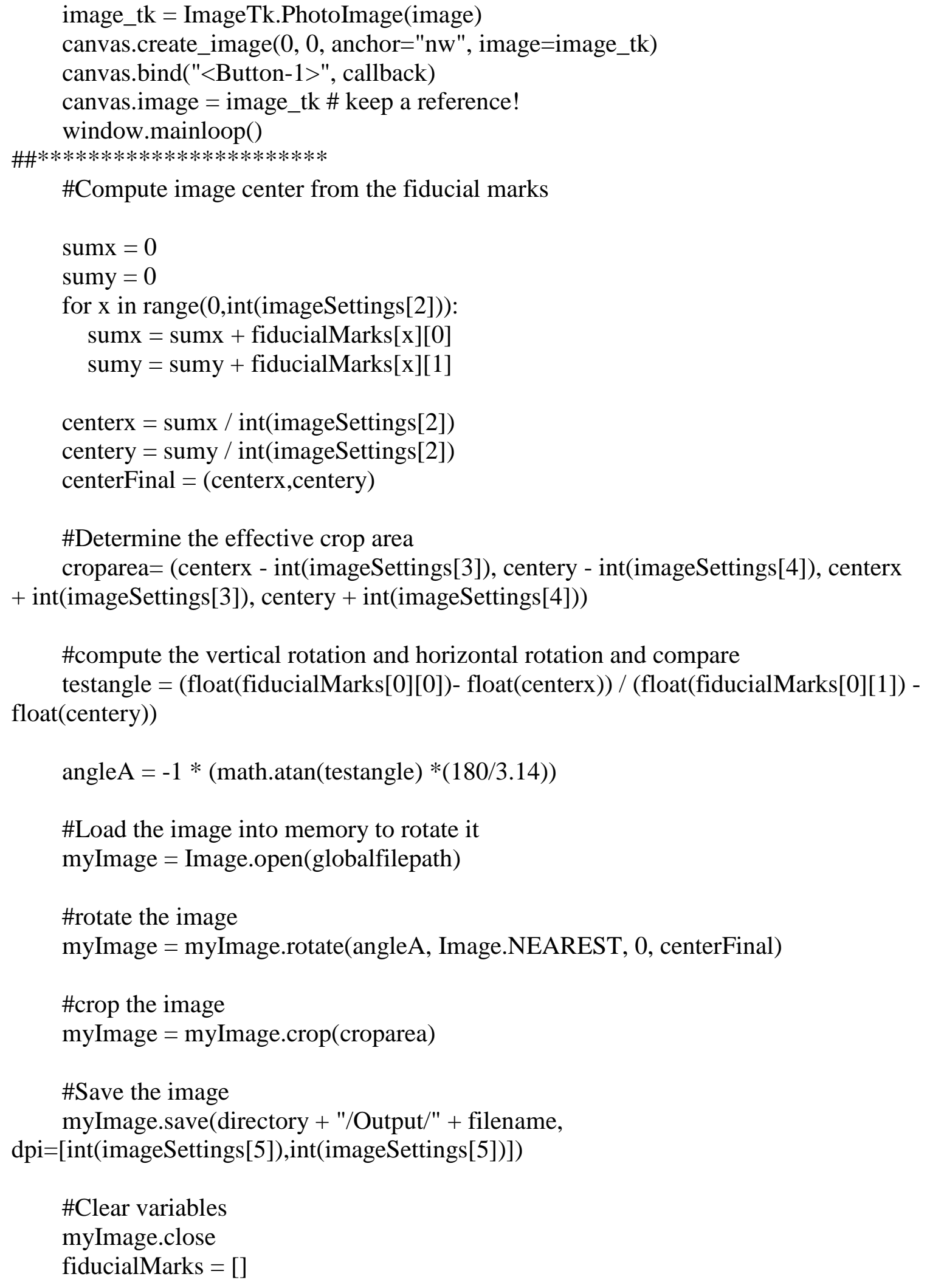

\#Compute image center from the fiducial marks

sumx $=0$

sumy $=0$

for $x$ in range(0,int(imageSettings[2])):

sumx $=$ sumx + fiducialMarks $[\mathrm{x}][0]$

sumy $=$ sumy + fiducialMarks[x][1]

centerx = sumx / int(imageSettings[2])

centery = sumy / int(imageSettings[2])

centerFinal $=($ centerx, centery $)$

\#Determine the effective crop area

croparea $=$ (centerx - int(imageSettings[3]), centery - int(imageSettings[4]), centerx

+ int(imageSettings[3]), centery + int(imageSettings[4]))

\#compute the vertical rotation and horizontal rotation and compare

testangle $=($ float $($ fiducialMarks[0][0]) - float $($ centerx $)) /($ float(fiducialMarks[0][1] $)$ float(centery))

angleA $=-1 *($ math.atan $($ testangle $) *(180 / 3.14))$

\#Load the image into memory to rotate it

myImage = Image.open(globalfilepath)

\#rotate the image

myImage = myImage.rotate $($ angleA, Image.NEAREST, 0 , centerFinal $)$

\#crop the image

myImage $=$ myImage.crop $($ croparea $)$

\#Save the image

myImage.save(directory + "/Output/" + filename, dpi=[int(imageSettings[5]),int(imageSettings[5])])

\#Clear variables

myImage.close

fiducialMarks = [] 